\title{
PERFORMANCE OF A BANDLIMITED TRELLIS DECODING RECEIVER FOR MSK CLASS MODULATIONS IN ADJACENT CHANNEL INTERFERENCE
}

\author{
by \\ James Harley \\ A Thesis \\ Presented to the Faculty of Graduate Studies \\ in Partial Fulfillment of the Requirements \\ for the Degree \\ MASTER OF SCIENCE \\ Department of Electrical \& Computer Engineering \\ University of Manitoba \\ Winnipeg, Manitoba
}

(c) March, 1996 
The author has granted an irrevocable non-exclusive licence allowing the National Library of Canada to reproduce, loan, distribute or sell copies of his/her thesis by any means and in any form or format, making this thesis available to interested persons.
L'auteur a accordé une licence irrévocable et non exclusive permettant à la Bibliothèque nationale du Canada de reproduire, prêter, distribuer ou vendre des copies de sa thèse de quelque manière et sous quelque forme que ce soit pour mettre des exemplaires de cette thèse à la disposition des personnes intéressées.

L'auteur conserve la propriété du droit d'auteur qui protège sa thèse. Ni la thèse ni des extraits substantiels de celle-ci ne doivent être imprimés ou autrement reproduits sans son autorisation. 
Name

Dissertation Abstracts International and Masters Abstracts International are arranged by broad, general subject categories. Please select the one subject which most nearly describes the content of your dissertation or thesis. Enter the corresponding four-digit code in the spaces provided.

\section{Electemin and Electrical}

\section{Subject Categories}

\section{THE HUMANITIES AND SOCIAL SCIENCES}

\begin{tabular}{|c|}
\hline $\begin{array}{l}\text { ts } \\
\text { tion Science } \\
\text { Science ..... } \\
\text { ommunications } \\
\text { Communication }\end{array}$ \\
\hline $\begin{array}{l}\text { nity College } \\
\text { um and Instruction } \\
\text { hildhood .......... } \\
\text { ary }\end{array}$ \\
\hline
\end{tabular}

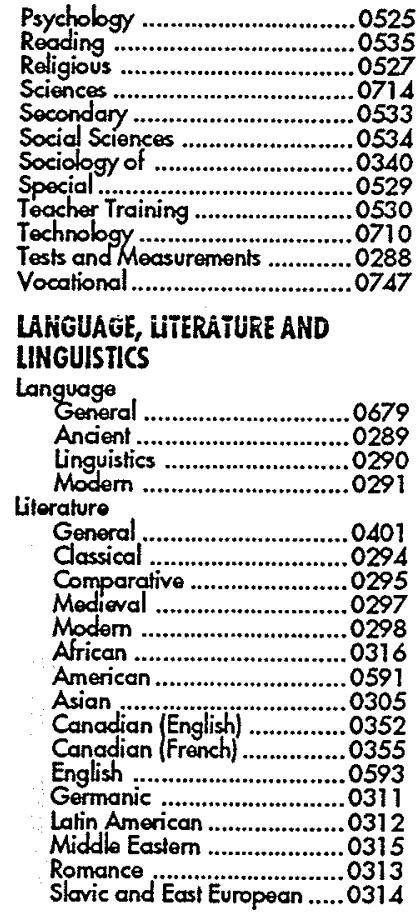

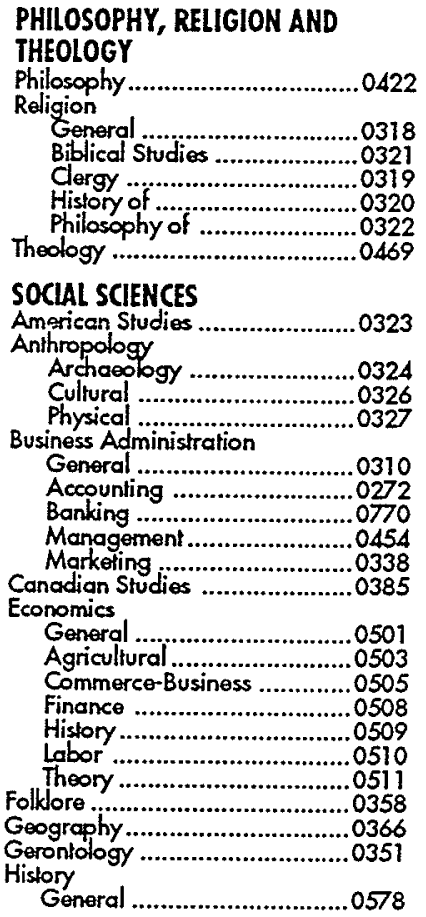

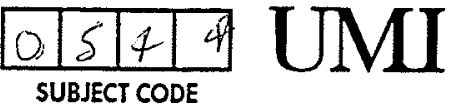

THE SCIENCES AND ENGINEERING

BIOLOGICAL SCIENCES

Agriculture

General ..............................0473

Agrotiomy ........................ 0285

Animal Culture and

Nutrition .........................0475

Animal Pathology ..................0476

Food Science and

Technology ...................0359

Forestry and Wildilife .............0478

Plant Culure ........................0479

Plant Physiology …….................0817

Range Management ..............0777

Wood Technology ...................0746

Gology

General .................................0306

Anatomy .................................0287

Biostatistics ..........................0308

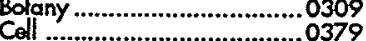

Ecology

Entomology ............................ 0353

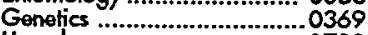

limnology ....................................0793

Microbiology ..........................0470

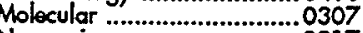

Neuroscience ...........................0317

Oceanogrophy .....................0416

Physiology …….....................0433

Radiation ............................082

Velerinary Seience...................0778

Zoology ….............................0472

Biophysics

General ................................0786

EARTH SCIENCES

Geochemistry..

.0425

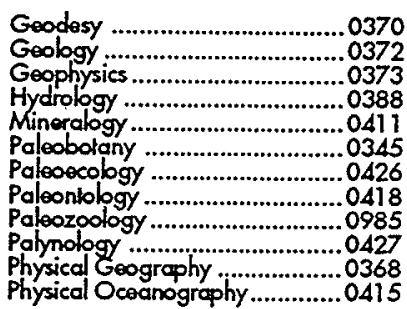

\section{HEALTH AND ENVIRONMENTAL}

\section{SCIENCES}

Environmental Sciences ..............0768

Health Seiences

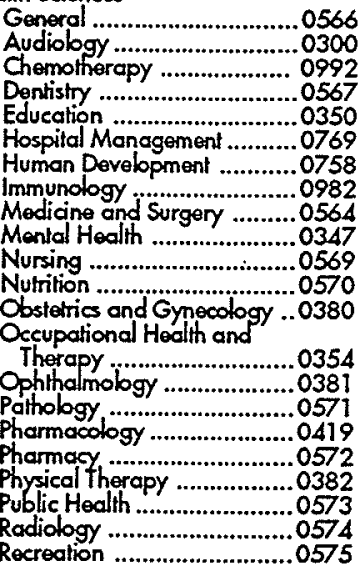

Speoch Pathology .................0460

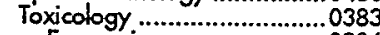
Home Economics ..........................0386

\section{PHYSICAL SQENCES}

\section{Pure Sciences}

Chemistry

General ...............................0485

Agricultural ................................0749

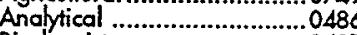

Biochemistry ..........................0487

Inorganie …...........................048

Nuctar ................................0738

Organic ...............................0490

Pharmaceutical ......................0491

Physical ................................0494

Polymer .................................0495

Mathematics .................................0405

Physics

General ..............................0605

Acoustics ..........................0986

Astronomy and

Astrophysics ....................0606

A Amospheric Science .............0608

Alomic............ Electricity .......0607

Elementary Particles and

High Energy.

Fluid and Plasma ..............0798

Molocular ............................0609

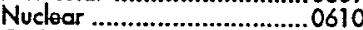

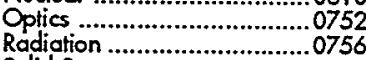

Radiation ............................0756

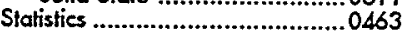

Applied Sciences

Applied Mochanics

Computer Science

.0346

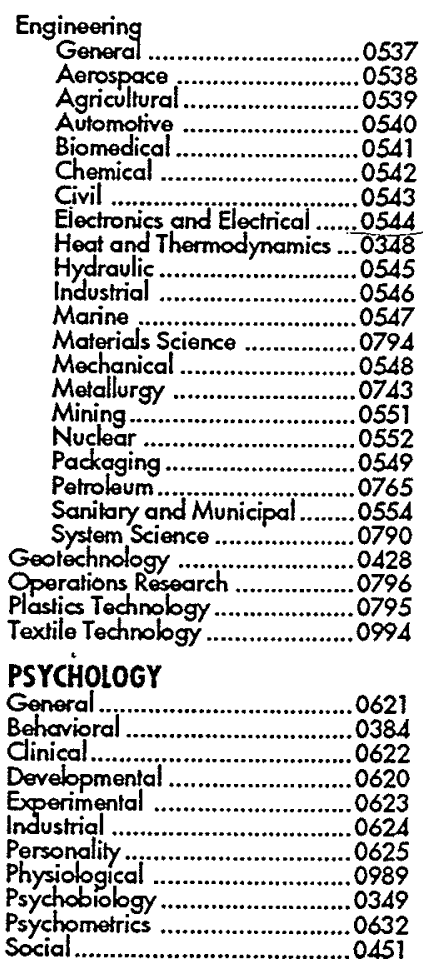

\begin{tabular}{|c|}
\hline 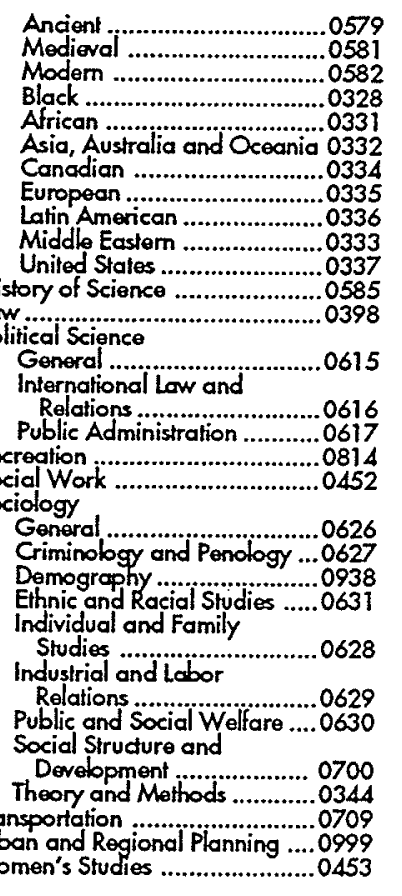 \\
\hline
\end{tabular}

Urban and Regional Planning ....0999 
CHANNEL INTERFERENCE

BY

JAMES HARLEY

A Thesis submitted to the Faculty of Graduate Studies of the University of Manitoba in partial fulfillment of the requirements of the degree of

MASTER OF SCIENCE

(C) 1996

Permission has been granted to the LIBRARY OF THE UNIVERSITY OF MANITOBA to lend or sell copies of this thesis, to the NATIONAL LIBRARY OF CANADA to microfilm this thesis and to lend or sell copies of the film, and LIBRARY MICROFILMS to publish an abstract of this thesis.

The author reserves other publication rights, and neither the thesis nor extensive extracts from it may be printed or other-wise reproduced without the author's written permission. 


\section{ACKNOWLEDGMENTS}

I wish to express my sincere gratitude to Dr. Ed Shwedyk for his constant guidance and encouragement throughout the M.Sc. program, and for the quality of both his scholarship and teaching. Grateful acknowledgment is also due to an NSERC scholarship for funding. Special thanks to Hongwei Kong, who as a valuable colleague, improved my learning with many stimulating discussions. In addition, I wish to thank Melissa for her support and encouragement throughout the entire M.Sc. experience. 


\begin{abstract}
The performance of a bandlimited trellis decoding receiver (BTR) is investigated for MSK class modulations in adjacent channel interference $(\mathrm{ACI})$. The receiver consists of a front-end bandlimiting filter, a Nyquist sampler, and Viterbi Algorithm decoding of the trellis matched to the sampled truncated impulse response of the channel. As there is no known optimum detector in non-Gaussian $\mathrm{ACI}$, this receiver is a candidate for superior performance in ACI compared to the matched filter (MF) receiver. Analysis as well as simulation results show that the BTR has indistinguishable performance compared to the optimum MF in additive white Gaussian noise only for Offset Quadrature Phase Shift Keying (OQPSK), Sinusoidal Frequency Shift Keying (SFSK) and Minimum Shift Keying (MSK). In ACI the BTR demonstrates considerable more robustness than the MF, suppressing up to $4.8 \mathrm{~dB}$ more ACI.
\end{abstract}


I hereby declare that I am the sole author of this thesis.

I authorize the University of Manitoba to lend this thesis to other institutions or individuals for the purpose of scholarly research.

James Harley.

I further authorize the University of Manitoba to reproduce this thesis by photocopying or by other means, in total or in part, at the request of other institutions or individuals for the purpose of scholarly research.

James Harley 


\section{TABLE OF CONTENTS}

1. INTRODUCTION

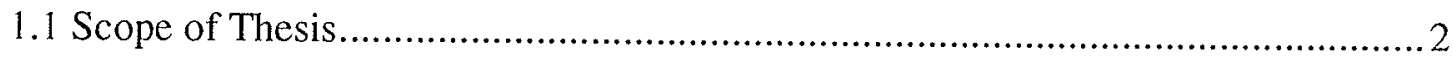

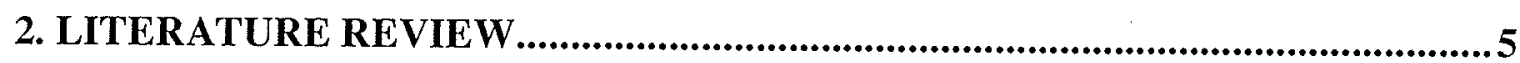

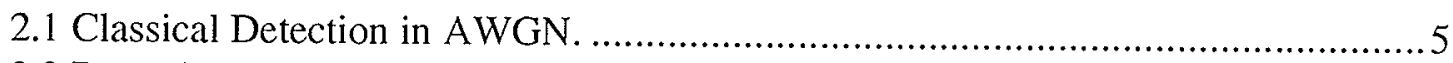

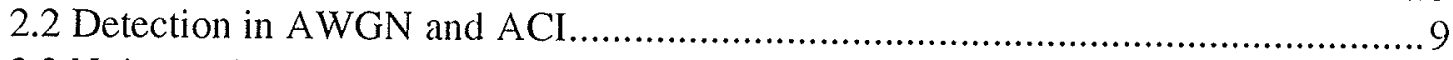

2.3 Nuisance Parameter............................................................................ 11

2.4 Joint Maximum Likelihood Sequence Estimation ........................................... 12

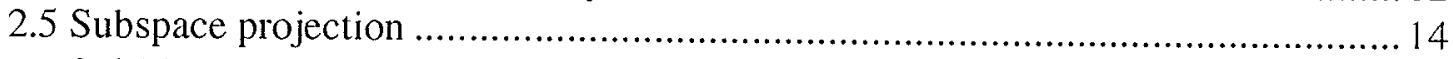

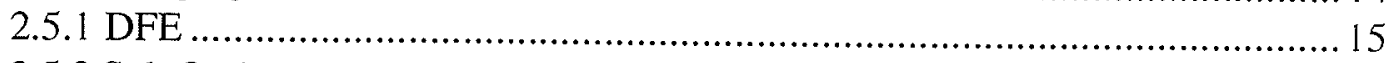

2.5.2 Sub Optimum MLSE in AWGN and ACI ........................................... 17

3. THE BTR FOR MSK CLASS MODULATIONS _............................................... 19

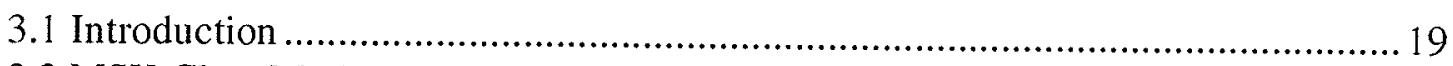

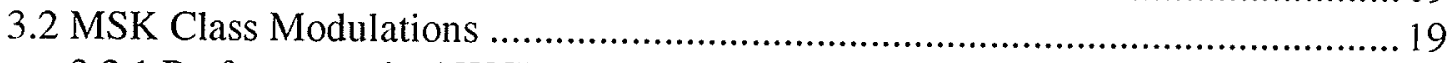

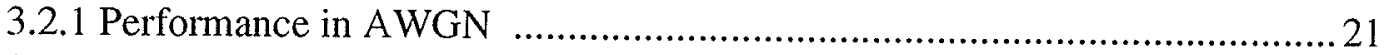

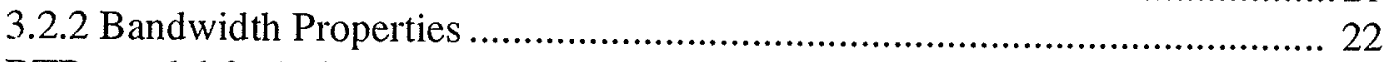

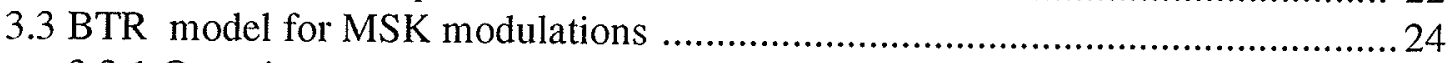

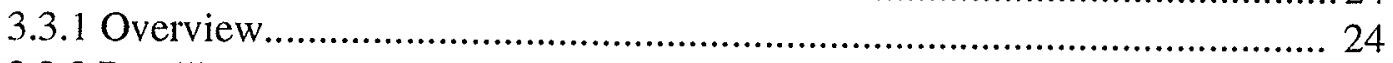

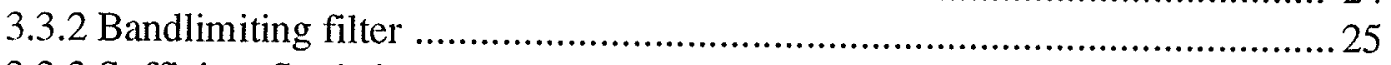

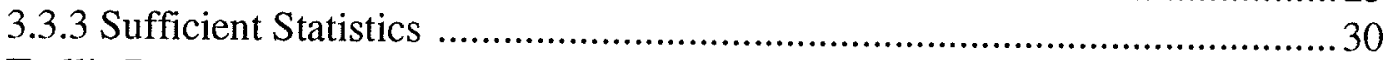

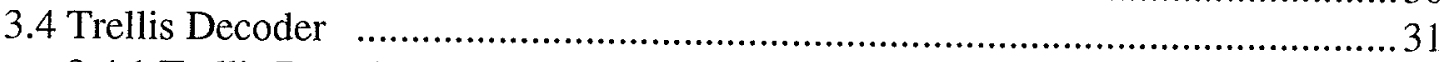

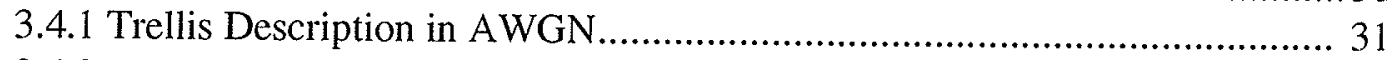

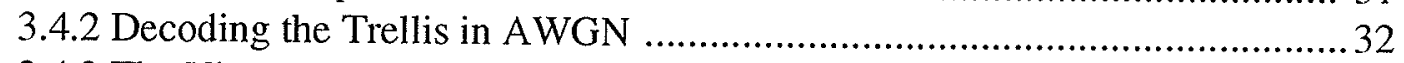

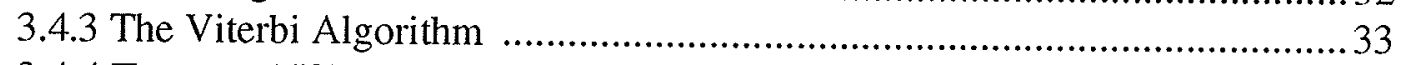

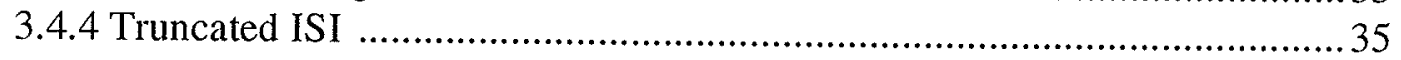

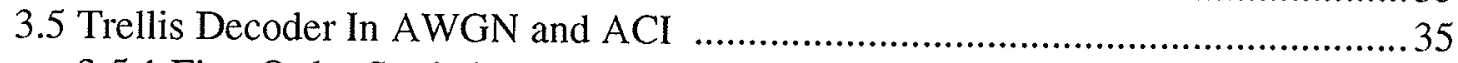

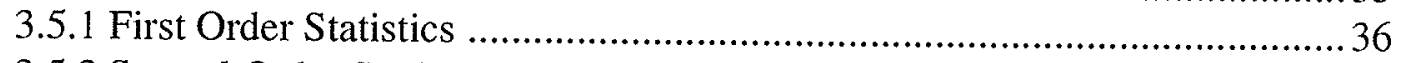

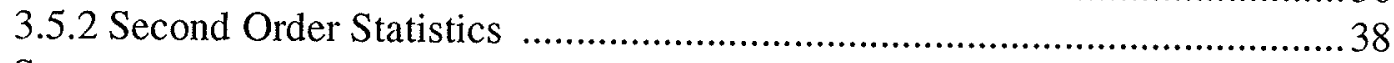

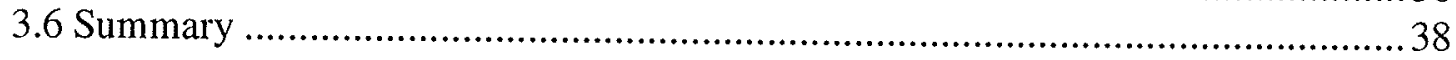

4. BTR PERFORMANCE IN AWGN AND ACI................................................39

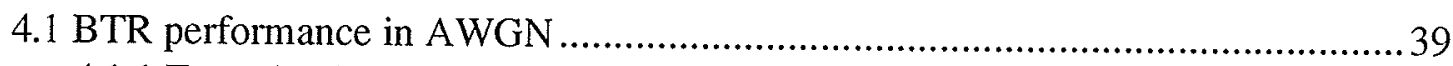

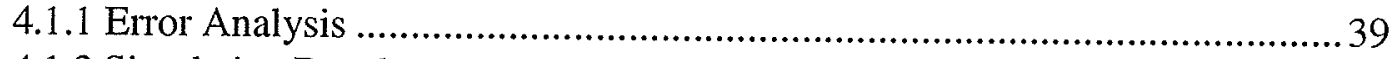

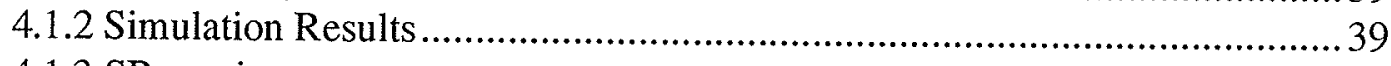

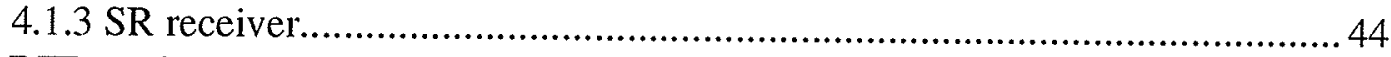

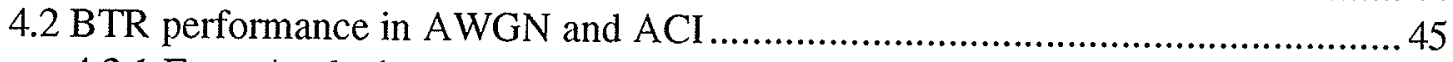

4.2.1 Error Analysis .............................................................................. 44

4.2.2 An example of the statistics of the filtered ACI ....................................4 47 
4.2.3 Simplified Analysis: Gaussian model

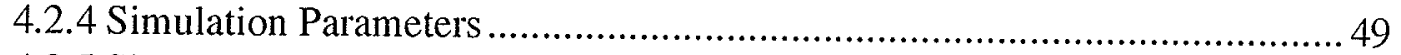

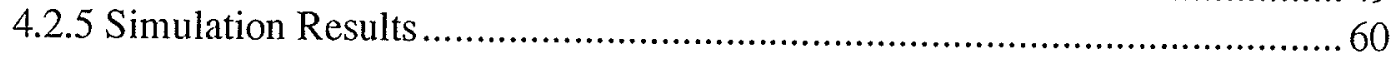

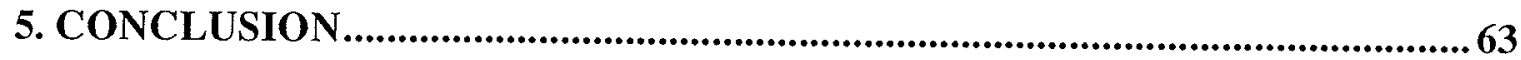

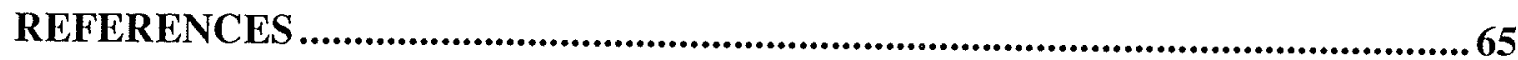




\section{LIST OF FIGURES}

Figure 3-1 Parallel implementation of a MSK modulator ........................................20

Figure 3-2 Quadrature Correlation filter for MSK modulations ..............................2 21

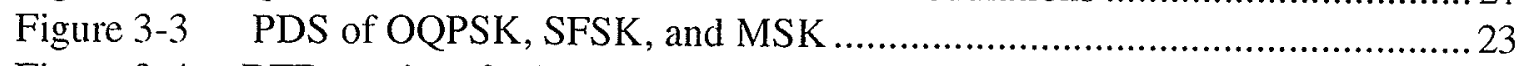

Figure 3-4 BTR receiver for MSK modulations .................................................. 24

Figure 3-5 Magnitude squared spectral response for the bandlimiting filter, $W=0.5 / T_{1}$, and for the matched filters for OQPSK and MSK ................................ 26

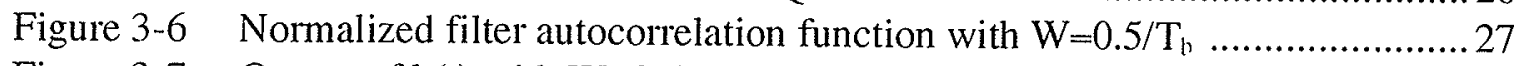

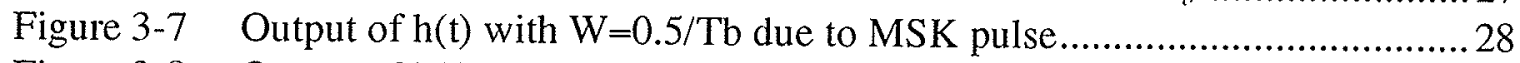

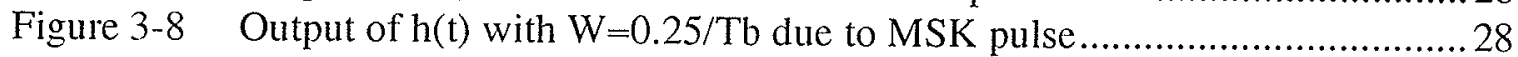

Figure 3-9 Output of $h(t)$ with $W=0.5 / \mathrm{Tb}$ due to adjacent MSK pulse frequency offset

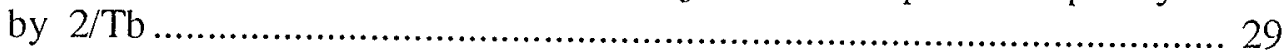

Figure 3-10 Output of $h(t)$ with $\mathrm{W}=0.25 / \mathrm{Tb}$ due to adjacent MSK pulse frequency

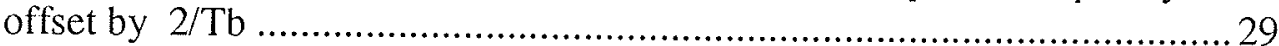

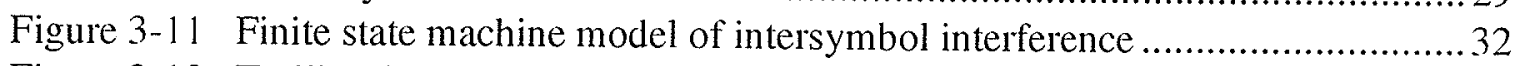

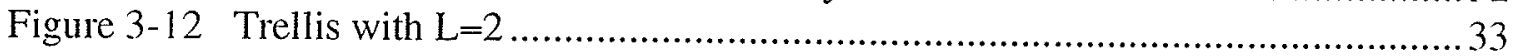

Figure 3-13 Estimate of amplitude density of filtered MSK pulse frequency offset at $2.0 / \mathrm{Tb}$ with $\mathrm{W}=0.5 / \mathrm{T}$ at $23 \mathrm{~dB}$ interference power to signal power, and Gaussian density with same variance as filtered ACI ..........................37

Figure 3-14 Estimate of amplitude density of filtered adjacent MSK pulse frequency offset at $2.0 / \mathrm{Tb}$ with $\mathrm{W}=0.25 / \mathrm{Tb}$ at $23 \mathrm{~dB}$ interference power to signal power....

Figure 4-1 BTR performance in AWGN for OQPSK, SFSK and MSK with $\mathrm{W}=0.5 / \mathrm{T}_{\mathrm{l}}$

Figure 4-2 BTR performance in AWGN for OQPSK, SFSK and MSK with $\mathrm{W}=0.25 / \mathrm{T}_{\mathrm{b}}$

Figure 4-3 Estimate of $\mathrm{f}\left\{\alpha=\right.$ alpha1) for adjacent MSK $\mathrm{f}$ offset at $2.0 / \mathrm{T}_{\mathrm{b}}$ at $20 \mathrm{~dB} \mathrm{I} / \mathrm{S} . .47$

Figure 4-4 Estimate of $f\left\{\hat{\alpha}=\right.$ alpha2) for adjacent MSK offset at $2.0 / \mathrm{T}_{\mathrm{b}}$ at $20 \mathrm{~dB} \mathrm{I} / \mathrm{S} \ldots 47$

Figure 4-5 OQPSK BTR $W=0.5 / T_{b}$ with one interferer at $f_{d}=2.0 / T_{h}$ and Gaussian model

Figure 4-6 OQPSK BTR $W=0.25 / \mathrm{T}_{\mathrm{b}}$, with one interferer at $\mathrm{f}_{\mathrm{d}}=2.0 / \mathrm{T}_{\mathrm{b}}$ and Gaussian model.

Figure 4-7 MSK BTR $W=0.5 / T_{b}$ with one interferer at $f_{d}=2.0 / T_{b}$ and Gaussian model

Figure 4-8 MSK BTR $W=0.25 / T_{b}$ with one interferer at $f_{d}=2.0 / T_{b}$ and Gaussian model.

Figure 4-9 $E_{b} /$ No dB loss compared to $M F$ with no ACI at $P_{c}=10^{-5}$ for one interferer at $f_{d}=2.0 / T_{b}$ versus $I / S d B$ for OQPSK

Figure 4-10 $E_{b} /$ No dB loss compared to MF with no $A C I$ at $P_{e}=10^{-5}$ for one interferer at $f_{d}=2.0 / T_{b}$ versus $I / S$ dB for MSK.

Figure 4-11 OQPSK BTR $W=0.5 / T_{b}$ with one interferer at $f_{d}=1.5 / T_{b}$ and Gaussian model.

Figure 4-12 OQPSK BTR $W=0.25 / T_{b}$ with one interferer at $f_{d}=1.5 / T_{b}$ and Gaussian model.... 
Figure 4-13 MSK BTR $W=0.5 / T_{b}$ with one interferer at $f_{d}=1.5 / T_{b}$, and Gaussian model.

Figure 4-14 MSK BTR W=0.25/ $\mathrm{T}_{\mathrm{b}}$ with one interferer at $\mathrm{f}_{\mathrm{d}}=1.5 / \mathrm{T}_{\mathrm{b}}$ and Gaussian model

Figure 4-15 $\mathrm{E}_{\mathrm{b}} / \mathrm{No} \mathrm{dB}$ loss compared to $\mathrm{MF}$ with no $\mathrm{ACI}$ at $\mathrm{P}_{\mathrm{c}}=10^{-5}$ for one interferer at $\mathrm{f}_{\mathrm{d}}=1.5 / \mathrm{T}_{\mathrm{b}}$ versus $\mathrm{I} / \mathrm{S} \mathrm{dB}$ for OQPSK

Figure 4-16 $\mathrm{E}_{\mathrm{b}} / \mathrm{No} \mathrm{dB}$ loss compared to $\mathrm{MF}$ with no $\mathrm{ACI}$ at $\mathrm{P}_{\mathrm{l}}=10^{-5}$ for one interferer at $\mathrm{f}_{\mathrm{d}}=1.5 / \mathrm{T}_{\mathrm{b}}$ versus $\mathrm{l} / \mathrm{S} \mathrm{dB}$ for MSK

Figure 4-17 OQPSK BTR $W=0.5 / T_{b}$ with one interferer at $f_{\mathrm{d}}=1.0 / T_{\mathrm{b}}$ and Gaussian model.

Figure 4-18 OQPSK BTR $W=0.25 / T_{\mathrm{b}}$ with one interferer at $\mathrm{f}_{\mathrm{d}}=1.0 / \mathrm{T}_{\mathrm{b}}$ and Gaussian model.

Figure 4-19 MSK BTR $W=0.5 / T_{1}$ with one interferer at $f_{d}=1.0 / T_{b}$ and Gaussian model.

Figure 4-20. MSK BTR $W=0.25 / T_{b}$, with one interferer at $f_{d}=1.0 / T_{1}$ and Gaussian model.

Figure 4-21 $\mathrm{E}_{\mathrm{l}} / \mathrm{No} \mathrm{dB}$ loss compared to MF with no $\mathrm{ACI}$ at $\mathrm{P}_{\mathrm{c}}=10^{-5}$ for one interferer at $f_{d}=1.0 / T_{1}$ versus $\mathrm{I} / \mathrm{S} \mathrm{dB}$ for OQPSK

Figure 4-22 $\mathrm{E}_{\sqrt{ }} / \mathrm{No}_{\mathrm{dB}}$ loss compared to $\mathrm{MF}$ with no $\mathrm{ACI}$ at $\mathrm{P}_{\mathrm{c}}=10^{-5}$ for one interferer at $f_{d}=1.0 / T_{b}$ versus $I / S d B$ for MSK.

\section{LIST OF TABLES}

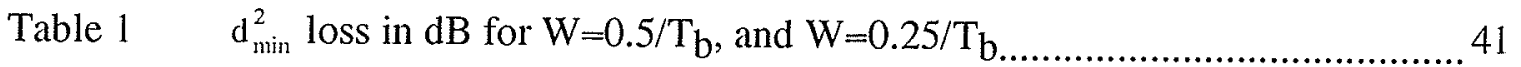

Table II $\quad S R d_{\min }^{2}$ loss in $\mathrm{dB}$ for $\mathrm{W}=0.5 / \mathrm{T}_{\mathrm{b}}$, and $\mathrm{W}=0.25 / \mathrm{T}_{\mathrm{b}}$

Table III I/S dB threshold for OQPSK, and MSK with one interferer at $f_{d} \ldots \ldots \ldots \ldots . . .61$

Table IV I/S dB threshold for the BTR with $\mathrm{W}=0.5 / \mathrm{T}_{\mathrm{b}}$ for OQPSK and MSK with one interferer at $f_{d}$ and the difference $(\Delta)$ in $d B$ to MF for same $f_{d,}$..... 61

Table $\mathrm{V} \quad \mathrm{I} / \mathrm{S} \mathrm{dB}$ Threshold for the BTR with $\mathrm{W}=0.25 / \mathrm{T}_{\mathrm{b}}$ for OQPSK and MSK with one interferer at $f_{d}$ and the difference $(\Delta)$ in $d B$ to $M F$ for same $f_{d}$ 


\section{INTRODUCTION}

In the ever crowded radio spectrum which demands closer spectral packing of signals, adjacent channel interference has become a serious limiting factor in Frequency Division Multiple Access (FDMA) systems. Furthermore high levels of adjacent channel interference $(\mathrm{ACI})$ can be experienced with the near-far problem, where a user receives a far distance signal in the proximity of someone else's transmission. For FDMA systems, the constant envelope minimum shift keying (MSK) type modulations is an attractive choice. The constant envelope constraint allows the use of less expensive, more power efficient nonlinear amplifiers in the transmission. Although the constant envelope implies a wider bandwidth compared to ideal Nyquist pulses, these constant envelope modulations still have attractive bandwidth properties with modulations such as MSK and Gaussian minimum shift keying (GMSK) .

A lot of the previous work in ACI suppression investigated the modulation question, culminating in the optimization of the baseband waveform to minimize the fraction of out of band power for a particular bandwidth [1]. Only recently has there been work on receiver design for improved performance in $\mathrm{ACI}$ compared to the standard quadrature correlation receiver (MF). This work [2]-[3] used wide front end filtering while sampling at twice the receiver's bandwidth in conjunction with either linear equalizer or decision feedback equalizers. For the case of one antenna with a signal in intersymbol interference (ISI) and ACI, this receiver showed some improvements in ACI suppression.

This thesis investigates the design and performance of a receiver for $\mathrm{ACI}$ suppression and additive white Gaussian noise (AWGN) mitigation. The approach taken is to strictly bandlimit the received signal and sample at the Nyquist rate to provide sufficient statistics of the bandlimited signal for a trellis decoder. The trellis decoder is matched to the truncated bandlimited signal and proceeds with a sub optimum maximum 
likelihood sequence estimation in the presence of AWGN and the filtered ACI. The receiver, called the bandlimiting trellis decoding receiver (BTR), rejects considerable $\mathrm{ACI}$ through narrow bandlimiting filtering and uses the ISI in the trellis decoding for detection. As there is no known optimum detector for non Gaussian ACI in AWGN, the BTR is a candidate for superior performance compared to the traditional matched filter (MF).

\subsection{Scope of Thesis}

The focus of this thesis is to investigate the performance of the BTR for MSK type modulations in AWGN and ACI. The performance of the BTR is benchmarked with the performance of the MF receiver, which is optimum in AWGN only. The thesis is composed as follows. Chapter two provides a review of classical detection theory for linear signals in AWGN and shows its limitations in the detection of signals in non Gaussian ACI. In addition, this chapter examines three sub-optimum approaches to this signal detection problem; the nuisance parameter approach, joint maximum likelihood sequence estimation (JMLSE), and subspace projection. The BTR is an example of subspace projection, where the received signal is projected onto a bandlimited subspace. Chapter three introduces the MSK type modulations as an example of linear offset quadrature modulation. This chapter then presents the BTR receiver for MSK type modulations, discussing in detail the bandlimiting filter and the resulting intersymbol interference of the signal of interest (SOI) and the statistics of the filtered ACI, as well as the metrics for the trellis decoder in AWGN and in ACI. The first section of Chapter four contains analysis and Monte Carlo simulation results for the performance of the BTR in AWGN compared to the optimum performance of the MF. The last section presents extensive Monte Carlo simulations for the performance of the BTR compared to the MF in AWGN and ACI, as well as a simplified analysis of the BTR based on modeling the 
filtered ACI as white Gaussian noise. The final chapter summarizes the results of the thesis and indicates further areas of study. 


\section{LITERATURE REVIEW}

This chapter first provides a review of classical detection theory in AWGN for linear signals (maximum likelihood detection) and then shows its limitations in the detection of signals in non Gaussian ACI. The review goes into some detail because establishing the vector space concepts is important in understanding the rest of the chapter. It also serves as background for the BTR, which performs MLSE on a bandlimited subspace. Three standard sub-optimum approaches, the nuisance parameter approach, joint maximum likelihood sequence estimation (JMLSE), and subspace projection are examined in the context of this signal detection problem. The nuisance parameter approach is shown to be untractable as a realizable receiver, while the JMLSE results in excessive hardware complexity. Both the fractionally spaced DFE and the BTR are discussed as examples of the subspace projection approach.

\subsection{Classical Detection in AWGN.}

The discussion focuses on the case of detecting linear signals in AWGN. For MSK class modulation, the transmitted signal can be considered as two linear antipodal signals in quadrature. Consider symbol by symbol detection, where the received signal for binary signaling is:

$$
r(t)=s_{i}(t)+n(t) \quad 0 \leq t \leq T \quad i=1,2
$$

where either hypothesis $s_{1}(t)$ or $s_{2}(t)$ is equally likely, and $n(t)$ is white Gaussian noise with spectral density $\mathrm{No} / 2$ watts/Hz. The maximum a posterior (MAP) decision rule, given $r(t)$, is to choose the hypothesis which is most likely, in other words, choose the hypothesis whose a posterior density function $\mathrm{f}\left\{\mathrm{s}_{\mathrm{i}}(\mathrm{t}) \mid \mathrm{r}(\mathrm{t})\right\}$ has the largest value. When 
the hypotheses are equally likely, this decision rule can be restated in terms of $f\left\{r(t) \mid s_{i}(t)\right\}$ when considering the Bayes rule, since:

$$
f\left\{s_{i}(t) \mid r(t)\right\}=\frac{f\left\{r(t) \mid s_{i}(t)\right\} P\left\{s_{i}(t)\right\}}{f\{r(t)\}}
$$

Both $P\left\{s_{i}(t)\right\}$ (the probability of hypothesis of si(t)) and $f\{r(t)\}$ have the same value for either hypothesis, hence choosing the hypothesis with largest $f\left\{r(t) \mid s_{i}(t)\right\}$, which is known as the maximum likelihood rule (ML), is equivalent to the MAP rule. In the continuous signal case, the density function $f\left\{r(t) \mid s_{i}(t)\right\}$ is clearly not well formulated. Following the Karhunen-Loeve approach, this density function can be well defined by converting the continuous signal $r(t)$ into a set of discrete values, called sufficient statistics [4].

The Karhunen-Loeve approach is to project $r(t)$ onto an arbitrary set of orthonormal functions $\left\{\varphi_{1}(t), \varphi_{2}(t), \ldots\right\}$ which span $L^{2}(T)$, under the constraint that the set of random variables $\left\{r_{j}\right\}$, where $r_{j}=\int_{0}^{T} r(t) \varphi_{j}(t) d t$, are uncorrelated with each other. Since the noise has Gaussian statistics, then each $r_{j}$ is Gaussian because the output of any linear operation (here integration) on a Gaussian process is Gaussian as well. Note that the Gaussian noise in this case is white, therefore any arbitrary set of orthonormal functions satisfy the Karhunen-Loeve condition. Hence the set of $\left\{\mathrm{r}_{\mathrm{j}}\right\}$ are independent Gaussian random variables with mean $s_{i, j}=\int_{0}^{T} S_{i}(t) \varphi_{j}(t) d t$ and variance No/2 watts. As the original signal $r(t)$ can be reconstructed from $\left\{r_{j}\right\}$, the decision rule can be equivalently applied to $\left\{r_{j}\right\}$. Since the $f\left\{\mathbf{r} \mid s_{i}\right\}$ are Gaussian densities, the decision rule can be more compactly implemented by taking the natural log of the ratio of these two densities and comparing the result to a threshold. This test is called the log likelihood test $\Lambda(\mathbf{r})$ and is defined here as:

$$
\Lambda(\mathbf{r})=\ln \left[f\left\{\mathbf{r} \mid s_{1}\right\} / f\left\{\mathbf{r} \mid s_{2}\right\}\right]
$$

if $\Lambda(\mathbf{r})>0$ choose $\mathrm{s}_{1}(\mathrm{t})$ as the transmitted signal

$$
\text { if } \Lambda(\mathbf{r})<0 \text { choose } \mathrm{s}_{2}(\mathrm{t}) \text { as the transmitted signal }
$$




$$
\Lambda(\mathbf{r})=\frac{1}{N o}\left[-\sum_{j=1}^{\infty}\left(r_{j}-s_{1, j}\right)^{2}+\sum_{j=1}^{\infty}\left(r_{j}-s_{2, j}\right)^{2}\right]
$$

where $r_{j}=\int_{0}^{r} r(t) \varphi_{j}(t) d t$ and $s_{i, j}=\int_{0}^{T} s_{i}(t) \varphi_{j}(t) d t$

Note that the arbitrary set of orthonormal functions in the case of AWGN can be chosen to minimize the number of dimensions to evaluate $\Lambda(\mathbf{r})$. For a signal set of two signals, up to two orthornormal basis signals can represent either two signals exactly as a linear combination of these basis signals. The two basis signals can be constructed from the original signal set according to the well known Gram-Schmidt procedure. The complete set of orthornormal signals is now the vector space of the direct sum of the two basis signals and its orthogonal complement. $\Lambda(\mathbf{r})$ can be evaluated up to these two basis dimensions, because the remaining dimensions are orthogonal to $s_{1}(t)$ and $s_{2}(t)$ and contribute zero in the inner product terms in $\Lambda(\mathbf{r})$.

An alternative way to evaluate $\Lambda(\mathbf{r})$ is to recognize that as $\left\{r_{j}, s_{i, j}\right\}$ are coefficients of an orthonormal set of functions. According to Parseval's theorem, the right-hand side can be equivalently computed by:

$$
\Lambda(\mathbf{r})=\frac{1}{N_{0}}\left[\int_{0}^{T}\left(r(t)-s_{1}(t)\right)^{2} d t-\int_{0}^{F}\left(r(t)-s_{2}(t)\right)^{2} d t\right]
$$

Equation (2.4) indicates that the receiver chooses the signal closest to the received signal in Euclidean space. Here the Euclidean space is defined as the set of finite energy complex signals over the $(0, T)$ interval, where the inner product and norm are defined by:

$$
\begin{aligned}
& \langle x(t), y(t)\rangle=\int_{0}^{T} x(t) \overline{y(t)} d t \\
& \|x(t)\|^{2}=\int_{0}^{T}|x(t)|^{2} d t
\end{aligned}
$$


where $\overline{\mathrm{z}}$ denotes the complex conjugate of $\mathrm{z}$. The log likelihood function can be simplified to:

$$
\Lambda(\mathbf{r})=\frac{1}{N_{0}}\left[-2 \int_{0}^{T} r(t) s_{1}(t) d t+2 \int_{0}^{T} r(t) s_{2}(t) d t+E_{1}-E_{2}\right]
$$

where $E_{i}=\int_{0}^{T} s_{i}^{2}(t) d t$ joules, for $i=1,2$, is the energy of $s_{i}(t)$.

Assuming the receiver knows the energy of the two signals $\left(E_{1}\right.$ and $\left.E_{2}\right)$, then the receiver requires only to perform two correlations with the received signal by using two filters with impulse responses $h_{i}(t)=s_{i}(T-t)$. These filters are "matched" to the signals $s_{1}(t)$ and $s_{2}(t)[4]$.

In the case of a sequence of statistically independent symbols, this maximum likelihood approach can be easily extended to maximum likelihood sequence estimation (MLSE). For binary antipodal signaling, then the received signal is:

$$
r(t)=\sum_{j=1}^{N} a_{j} p(t-j T)+n(t)
$$

where $p(t)$ is an arbitrary waveform defined over $(0, T)$ outside of which it is zero, and $a_{j}$ are equally likely $(1$ or -1$)$ independent random variables. The approach here is to determine the most likely sequence of $\mathrm{N}$ symbols (or equivalently the $1 \mathrm{xN}$ vector $\mathbf{a}$ ) given $r(t)$. Hence MLSE means choosing a that has largest $\ln [f\{r \mid \mathbf{a}\}]$ since each sequence is equally likely. According to the approach outlined above, then

$$
\Lambda(\mathbf{r})=\frac{1}{N_{0}} \int_{0}^{N J}\left(r(t)-\sum_{j=1}^{N} a_{j} p(t-j T)\right)^{2} d t=\frac{1}{N o} \sum_{j=1}^{N} \int_{(j-1) T}^{j r}\left(r(t)-a_{j} p(t-j T)^{2} d t\right.
$$

The vector space concepts are now naturally extended to $(0, N T)$ space. Thus the MLSE receiver chooses the signal sequence closest to the received signal over 0 to NT. Notice that in this case because each symbol is statistically independent from each other and there 
is no time overlap between symbols, the MLSE collapses down to symbol by symbol decisions.

\subsection{Detection in AWGN and ACI}

It is still an open question to find a method to generate a set of orthonormal functions such that the resulting $f\{\mathbf{r} \mid \mathbf{a}\}$ in AWGN and ACI leads to a realizable receiver structure. The Karhunen-Loeve expansion method fails in the presence of ACI and AWGN, because of the non-Gaussian statistics of the ACI. Assuming the ACI is wide sense stationary, then even if a set of orthonormal functions are found that satisfy the Karhunen-Loeve condition, it does not follow that the uncorrelated projections on each of these orthornormal functions are statistically independent. Although the joint density function $f\{\mathbf{r} \mid \mathbf{a}\}$ could be determined in theory, there is no guarantee that the resulting $f\{\mathbf{r} \mid \mathbf{a}\}$ is implementable. Just as the Karhunen-Loeve method is specialized for the additive Gaussian noise case, it is possible that there are specialized methods that apply to certain types of ACI, but up to this date no research has been conducted along these lines.

In general, for the optimum detection of signals in ACI and AWGN, the interpretation of choosing the signal closest to the received signal in Euclidean space has to be abandoned. There is the important case when the ACI is designed to be orthogonal to the space spanned by the signal set of interest (SOI). If the received waveform in this case is projected onto a vector space which is spanned by the SOI then the resulting sufficient statistics have no component due to $\mathrm{ACI}$. The detection problem reduces to the case of detecting the SOI in AWGN. Hence in this case the vector space interpretation can be kept.

Many modulation schemes have been designed to meet this requirement of orthogonality. There are two main obstacles to achieving orthogonality, phase offset and symbol time offset between the SOI and the adjacent channels. Phase offset is the relative 
phase difference between carriers, and symbol time offset is the difference in time that a symbol is asserted. The simplest case is when the channels are phase offset but symbol time synchronized. Examples of this case are Frequency Division Multiple Access (FDMA) and Code Division Multiple Access ( CDMA). A synchronized FDMA system divides up its frequency band into equal sized bands which are spaced such that noncoherent orthogonality is obtained. Typically FDMA systems use quadrature phase shift keying (QPSK) which requires a 1/T frequency spacing. A synchronized CDMA system on the other hand shares its frequency band with all channels, and ensures orthogonality by supplying each user with an orthogonal spreading code ( i.e, orthogonal to all the other codes).

The requirement to be orthogonal for all symbol time offsets say between $x(t)$ (the $\mathrm{SOI}$ ) and $\mathrm{a}(\mathrm{t})$ ( the $\mathrm{ACI}$ ) is harder to achieve. In effect, this means the cross correlation between $x(t)$ and $a(t)$ is zero for all time shifts. In other words,

$$
\int_{-\infty}^{\infty} x(t) a(t+\tau) d t=0 \quad \text { for all } \tau
$$

This cross correlation is simply the convolution of $x(t)$ with a(- $t)$. This condition translated to the frequency domain means that the product $X(f) \bar{A}(f)$ equals zero for all frequencies, where $X(f)$ and $A(f)$ are the Fourier transforms of $x(t)$ and $a(t)$ respectively. Therefore $X(f)$ and $A(f)$ have to be frequency limited in a complementary manner to achieve this condition. One method is for $\mathrm{X}(\mathrm{f})$ and $\mathrm{A}(\mathrm{f})$ to be bandlimited and sufficiently frequency separated so there is no frequency overlap. Hence, the traditional notion of adjacent bandlimited channels comes naturally out of this discussion. Note that frequency limitation implies an infinite time span for each symbol signal which adds the complication of detecting a sequence of symbols which overlap with each other. One solution to this classic problem of detecting a sequence of bandlimited signals without intersymbol interference is the set of Nyquist pulses . 
There are number of situations where non-orthogonal ACI is unavoidable. Constant amplitude modulations like MSK are desired when using amplifiers in the more power efficient non-linear region, or the channel distortion is such, as in fast fading mobile channels, that the orthogonality is destroyed resulting in ACI. There are at least three sub optimum approaches for the receiver design in the presence of unwanted ACI. These are the nuisance parameter, joint estimation, and projection into subspace approaches, each of which are discussed briefly.

\subsection{Nuisance Parameter}

The nuisance parameter approach has been successfully applied to unwanted random parameters in the SOI itself such as phase uncertainty or amplitude variation due to flat fading channels [5]. It has been suggested that this approach could be applied to ACI, which would be modeled as an additive unwanted parameter [6]. The following discussion shows that this approach is only successful, but unwieldy, in the case of synchronized and noncoherent ACI, and fails in the more general unsynchronized scenario.

For simplicity in the discussion, let the received signal in AWGN and ACI be:

$r(t)=s(t)+a(t)+n(t)$
$s(t)=\sum_{j=1}^{N} b_{j} p(t-j T)$
$a(t)=\sum_{j=1}^{N} c_{j} p(t-j T-\lambda) \cos \left(2 \pi f_{d} t+\theta\right)$

where $b_{i}, c_{i}$ are independent equally likely (1 or -1) random variables, and the synchronization offset and the phase offset $\lambda, \theta$ are uniform independent random variables over $(0, T)$ and $(0,2 \pi)$ respectively. The nuisance parameter approach is to assume $a(t)$ is known in order to form $\mathrm{f}\{\mathbf{r} \mid \mathbf{b}, \mathbf{c}, \lambda, \theta\}$ using the standard Karhunen-Loeve method for 
AWGN. Then the desired $f\{\mathbf{r} \mid \mathbf{b}\}$ is obtained by averaging out the random parameters c, $\tau, \theta$ according to the joint density function $\mathrm{f}\{\mathbf{c}, \lambda, \theta\}=\mathrm{P}\{\mathbf{c}\} \mathrm{f}\{\lambda\} \mathrm{f}\{\theta\}$. In equation form:

$$
\begin{gathered}
f\{\mathbf{r} \mid \mathbf{b}\}=\sum_{i=1}^{N} P\left\{c_{i}\right\} \int_{\lambda \theta} f\{\lambda\} f\{\theta\} f\{\mathbf{r} \mid \mathbf{b}, \mathbf{c}, \lambda, \theta) d \theta d \lambda= \\
\left.\sum_{i=1}^{N} \frac{P\left\{c_{i}\right\}}{(\pi N o)^{1 / 2}} \int_{0}^{2 \pi} \int_{0}^{2 \pi} \exp \left\{-\frac{1}{N_{0}} \int_{0}^{N r}\left[r(t)-\sum_{j=1}^{N} b_{i} p(t-j T)+\sum_{j=1}^{N} c_{j} p(t-j T-\lambda) \cos \left(2 \pi f_{d} t+\theta\right)\right\}\right]^{2} d t\right\} d \theta d \lambda
\end{gathered}
$$

The nuisance parameter approach results in the best average receiver in the case where theset of random ( or nuisance ) parameters are not estimated or detected. For the general case of an unsynchronized adjacent signal, this expression is not tractable for a realizable receiver. There is the complication of correlation between symbols due to time overlap of the adjacent signal's symbols, but more detrimentally is the non tractable (analytically) integral which does not provide the form for the sufficient statistics required by the receiver. In the case that the adjacent channel is synchronized $(\lambda=0)$, this expression could be analyzed for a realizable receiver. This case is uninteresting, since if the adjacent channel is synchronized, then a noncoherently orthogonal modulation scheme for the two channels would be chosen resulting in no ACI.

\subsection{Joint Maximum Likelihood Sequence Estimation}

Joint maximum likelihood sequence estimation (JMLSE) is an optimum strategy for detecting both the SOI and the ACI in AWGN. The JMLSE receiver chooses one ensemble member of the SOI and ACI stochastic process that is closest in Euclidean distance to the received signal. Although JMLSE is not necessarily optimum for detecting 
the SOI alone in AWGN and ACI, for the case where the cross-correlation between the SOI and the ACI is relatively weak, the performance can be very close to the performance of detecting the SOI in AWGN without ACI[7]. Since there is correlation between symbols, due to symbol overlap between adjacent channels because of the synchronization offsets, the JMLSE does not simplify to symbol by symbol detection. The main drawback for the JMLSE approach is the large increase in hardware, such as the additional phase lock loops, frequency oscillators and matched filters for detecting the adjacent channel signals. This increase in hardware may be prohibitive in some applications, such as battery powered hand held communication devices. Also there is some complexity involved in computing the JMLSE from the sufficient statistics with a dynamic programming algorithm such as the Viterbi Algorithm (VA).

One important feature of the JMLSE is its performance in the near-far effect, where the ACI is at a much higher power level than the SOI. Under the assumption that the phase and synchronization offsets of the ACI are known to the receiver, the JMLSE performance is neither limited and probably not even degraded, as is the case with the conventional matched filter receiver, by any relative increase in the power of the ACI. The JMLSE performance is not limited because there is no unbounded nuisance parameter due to the $\mathrm{ACI}$ as there is with the conventional receiver, since all of the $\mathrm{ACI}$ is estimated. In fact, with the increase in relative power of the ACI, the estimate of the ACI becomes more reliable. For the conventional receiver, however, an increase in $\mathrm{ACI}$ power means that the $\mathrm{ACI}$ at the output of the matched filter is more detrimental to performance. That the JMLSE performance may not even be degraded in the near far situation is suggested by some simulation studies of asynchronous CDMA systems detected by JMLSE [7]. The asynchronous CDMA system is analogous to ACI in the sense that each user's signal is weakly correlated with each other over all synchronization offsets. The results show that for the simple case of two CDMA signals there is an improvement in performance where, with an increase in the other user's signal power, the performance converges to that of 
a single user. Note, however, that this discussion applies in the idealistic case where the phase and synchronization offsets of the ACI are known to the receiver. In practice, one associates a density function with the error of the estimates of synchronization and phase

offsets $\hat{\lambda}$ and $\hat{\theta}$ which degrade the theoretical performance. The performance behavior of the JMLSE in the near-far effect should be confirmed under a realistic assumption for $\mathrm{f}\{\hat{\lambda}\}$ and $\mathrm{f}\{\hat{\theta}\}$.

Another difficulty with JMLSE for ACI in FDMA systems is exactly how much ACI should be jointly estimated. For example, should just the two closest adjacent channels be estimated, in which case there is some unestimated ACI, or should all the channels be estimated, which is prohibitively expensive in hardware. It is not clear that jointly estimating three channels in an unsynchronized FDMA system should have better performance for the SOI then simply detecting the SOI with a bandlimiting filter. One area where JMLSE has found application is in jointly estimating the SOI in cochannel interference, where the interference is localized in time and frequency with the SOI[8].

\subsection{Subspace projection}

Since the projection of the SOI and ACI onto a complete space is undesirable due to prohibitive receiver hardware and complexity, an alternative approach is to project onto a reduced subspace that is chosen according to an acceptable criterion. One problem is that there is no tractable criterion in the presence of ACI related to error performance. Note that for the case of stationary Gaussian ACI, the optimum filter (or equivalently the subspace) for the detection of the SOI is the whitened matched filter which also maximizes the signal power to interference power ratio (S/I). It is generally accepted that the best criterion in lieu of the ideal criterion is the subspace that maximizes the $\mathrm{S} / \mathrm{I}$. The difficulty, however, is in implementing this criterion, since the filtering is band limited which causes 
both intersymbol interference and noise correlation. There is an inherent trade off between $\mathrm{ACI}$ rejection and ISI. The more bandlimited the filter is, the better the $\mathrm{ACI}$ rejection is, but the resulting ISI is more severe.

Because of the difficulties in implementing this criterion, there has only been one example where this criterion has been applied directly[9]. In this case the filtered received signal is sampled at the symbol rate and a decision feedback equalizer (DFE) is employed to reduce the resulting intersymbol interference. Other research has avoided ISI by optimizing a filter under the S/I criterion with the constraint that the filter has a limited time span[10]. Another study used[11] spectrally tighter filters but do not equalize the resulting ISI, since the increased ACI rejection more than offset this performance loss due to ISI.

In the last two decades there has been significant progress made in equalizing ISI resulting from the channel or transmitter filters. The two key areas are the DFE and the optimum MLSE approaches. Gains in ISI equalization are directly translated to gains in ACI rejection by allowing spectrally tighter filters at the front end of the receiver without comparative ISI loss. The rest of the chapter reviews how the DFE has been extended to combat ACI, and then presents a sub-optimum MLSE approach to combat ACI, which is the focus of this thesis.

\subsubsection{DFE (Decision Feedback Equalization)}

The DFE was originally devised as a modification to linear equalization of ISI channels. The DFE is composed of a forward filter, a sampler, and a decision threshold followed by a discrete feedback filter. The forward filter is designed to substantially frequency equalize the ISI, but with less noise enhancement than the perfect frequency equalization of the linear equalizer, with the residual ISI largely removed by the feedback 
filter. Typically the forward and feedback filter are chosen according to the minimum mean square error criterion (MMSE), which can be easily implemented adaptively using the least mean square algorithm (LMS).

The last decade has seen intense research into extending the DFE to combat $\mathrm{ACI}$ and co-channel interference(CCI) as well as ISI [2],[12]. Many simulation results confirm that fractionally spaced DFE, where the sampler samples atat least twice the signalling rate, is more robust than traditional MF receivers in suppressing ACI and CCI [13]. There are two explanations for the robust performance of fractionally spaced DFE. The first is that the sampled frequency response of the SOI is no longer aliased, and thus the forward filter can be more discriminating in suppressing interference, allowing the feedback filter to reduce the ISI. The second explanation is that choosing the forward filter under the MMSE criterion for two samples per symbol time exploits the cyclostationary nature of the interference. Since the SOI and the ACI are modulated signals, they are cyclostationary stochastic processes, where their mean and autocorrelation functions are periodic with time. Recent research has shown that the classical MMSE filtering (or Wiener filtering) for signals in interference can be extended to cyclostationary signals (or cyclic Wiener filtering) [14]. In the case of modulated signals, there is some gain in cyclic Wiener filtering compared to Wiener filtering, where the modulated signals are modeled as wide sense stationary by assuming the phase of the carrier is a uniform random variable. Forcing the forward filter to minimize the mean square error for two samples per symbol time means the forward filter more closely approximates the cyclic Wiener filter that minimizes the instantaneous (in time) mean square error, not the average mean square error (over a period in time) which the traditional Wiener filter implements. In the context of narrowband signals considered here, however, the gains in cyclic Wiener filtering are marginal [15] and also require that adjacent signals maintain a fixed phase and synchronization offset which is not the typical situation. However, in synchronous wideband systems such as CDMA, though, cyclic Wiener filtering (and sub optimum 
implementations thereof) as a means to suppress CCI are proving to be a fertile research area $[16]$.

One disadvantage of DFE is that the symbol by symbol hard decisions which are incompatible with soft decision decoding. Soft decision decoding, where a sampled version of the received signal is retained, is used for convolution codes, and trellis coded modulations in general. Although a form of DFE (Predictive Decision-Feedback - Equalizer) has been designed for soft decision decoding, where the estimated bits from the decoding algorithm (usually the Viterbi algorithm) feed the feedback filter to reduce the ISI, the implementation is sub optimum since the forward and feedback taps are not jointly optimized under the MMSE criterion [17]. Another disadvantage is that the energy of ISI resulting from the forward filter is ignored in the detection of the signal. For signals in general, coded or uncoded, the ISI can be incorporated into the signal as a trellis and be detected using a soft-decision decoding algorithm for some detection gain.

\subsubsection{Sub Optimum MLSE in AWGN and ACI}

Maximum likelihood sequence estimation of signals in intersymbol interference is an optimum detection strategy. MLSE detection of signals in finite ISI is made tractable by modeling the ISI as a finite state machine (or equivalently a trellis) and evaluating the MLSE metric recursively using the Viterbi algorithm (VA.) [18]. The MLSE approach was originally realized for linear modulations with a whitened matched filter which provided sufficient statistics as well as decorrelating the noise [19]. Recently the MLSE approach has been applied to trellis coded modulation (non linear modulations), whereby a bandlimited filter followed by a Nyquist sampler provides the uncorrelated sufficient 
statistics [20]. For any given ISI channel, MLSE always performs better than DFE, because the MLSE uses the energy of the ISI for a detection gain. Depending on the nature of the ISI, the performance difference can be significant [17].

There has been no explicit work in using MLSE methods to combat ACI. Simmons proposed a bandlimiting trellis decoding receiver (BTR) using low pass filters followed by Nyquist samplers for continuous phase modulations (CPM) as a way to reduce the number of matched filters [21],[22]. The significant ISI terms are mapped onto the modulation trellis, which is then decoded by a trellis decoding algorithm such as the VA or Malgorithm. His work shows that with low pass filters of spectral roll off comparable to the CPM scheme, the BTR has the same ACI rejection capacity as the full matched filter VA receiver. The BTR can be extended to combat ACI directly, by choosing low pass filters with steeper spectral roll off than the matched filter which should provide better ACI rejection capacity without any ISI penalty in performance.

The focus of this thesis is to investigate the performance in AWGN and ACI of the BTR for MSK class modulations viewed as a linear offset quadrature modulation. The lowpass filters used are very close to the ideal brickwall filters. The BTR thus performs a MLSE of a bandlimited space in AWGN only. It should be the noted that the BTR can be easily extended to non linear modulation, such as the CPM example from Simmons' work. 


\section{THE BTR FOR MSK CLASS MODULATIONS}

\subsection{Introduction}

This chapter initially discusses the class of MSK modulations in terms of the modulator, the optimum receiver in AWGN the quadrature matched filter, and the different types of MSK modulations with their respective spectral properties. The BTR is then developed in detail for the MSK class modulations, where the bandlimiting filter and the trellis decoding algorithm are presented for both cases of AWGN and AWGN with ACI.

\subsection{MSK Class Modulations}

Although MSK type modulations can be described as an example of a continuous phase modulation, where the continuity in the phase introduces memory into the signal, the discussion uses the alternative but equivalent model of linear quadrature constant envelope modulations. In this model, the transmitter divides the bit stream a into inphase and quadrature streams $\mathbf{a}^{\mathrm{I}}$ and $\mathbf{a}^{\mathrm{Q}}$, which linearly modulate the quadrature branches as shown in Figure 3-1.

The transmitted signal $\mathrm{s}(\mathrm{t})$ is:

$$
\begin{gathered}
s(t)=A \sum_{k} a_{k}^{1} p\left(t-k 2 T_{b}\right) \cos \left(2 \pi f_{c} t+\theta\right)+A \sum_{k} a_{k}^{Q} p\left(t-k 2 T_{b}-T_{b}\right) \sin \left(2 \pi f_{c} t+\theta\right) \\
A=\sqrt{2 E_{b} / T_{b}}
\end{gathered}
$$

where the MSK type pulse $p(t)$ satisfies the following constraints to ensure a constant envelope:

$$
\begin{gathered}
p(t)=p(-t) \\
p(t)^{2}+p\left(t-T_{b}\right)^{2}=1 \quad t \in\left(0, T_{b}\right)
\end{gathered}
$$




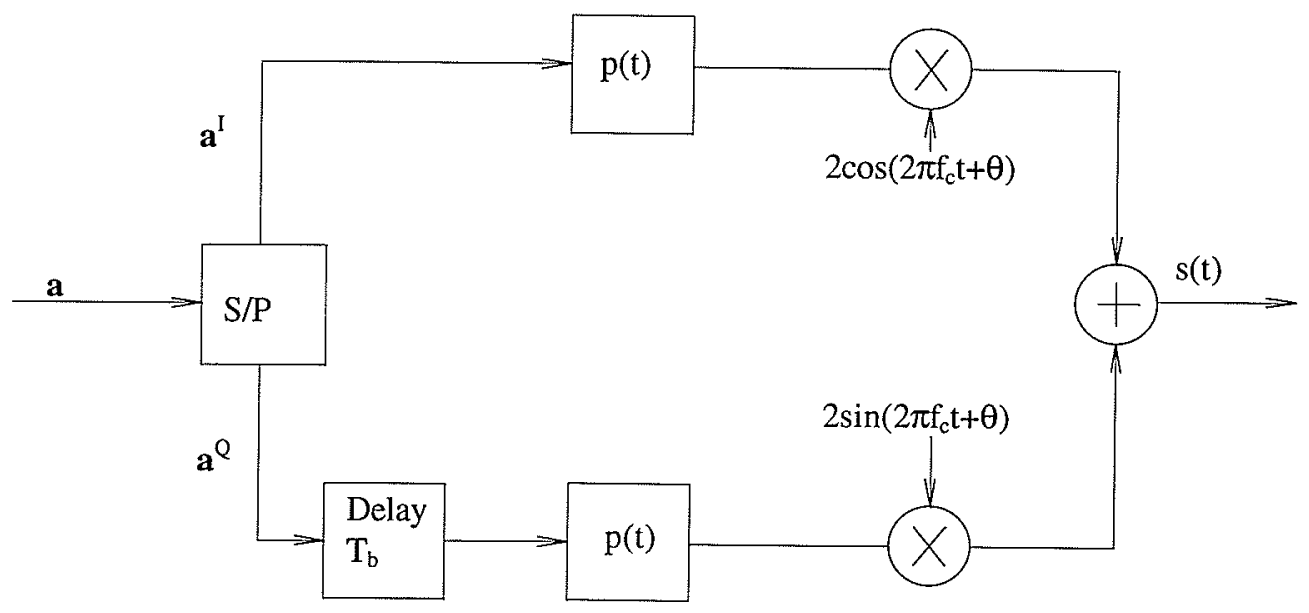

Figure 3-1 Parallel implementation of a MSK modulator

Note that the quadrature signals are interleaved by $\mathrm{T}_{\mathrm{b}}$ in order to obtain the constant envelope.

For coherent modulation where $\theta$ is assumed to equal zero, it is convenient to express $s(t)$ in terms of the baseband complex envelope $\hat{s}(t)$, where:

$$
\begin{gathered}
s(t)=\operatorname{Re}\left\{\hat{s}(t) e^{j 2 \pi f_{c} t}\right\} \\
\hat{s}(t)=A \sum_{k} a_{k}^{I} p\left(t-k 2 T_{b}\right)-j A \sum_{k} a_{k}^{Q} p\left(t-k 2 T_{b}-T_{b}\right)
\end{gathered}
$$

Three examples of MSK type modulations are offset quadrature phase shift keying (OQPSK), minimum shift keying (MSK) and sinusoidal frequency shift keying ( SFSK). Their pulse shapes have the following form:

$$
\begin{gathered}
\text { OQPSK: } \mathrm{p}(\mathrm{t})=\frac{1}{\sqrt{2} \mathrm{~T}_{\mathrm{b}}} \quad-\mathrm{T}_{\mathrm{b}} \leq \mathrm{t} \leq \mathrm{T}_{\mathrm{b}} \\
\text { SFSK: } \mathrm{p}(\mathrm{t})=\frac{1}{\mathrm{~T}_{\mathrm{b}}} \cos \left(\frac{\pi \mathrm{t}}{2 \mathrm{~T}_{\mathrm{b}}}-\frac{1}{4} \sin \left(\frac{2 \pi \mathrm{t}}{\mathrm{T}_{\mathrm{b}}}\right)\right) \quad-\mathrm{T}_{\mathrm{b}} \leq \mathrm{t} \leq \mathrm{T}_{\mathrm{b}}
\end{gathered}
$$




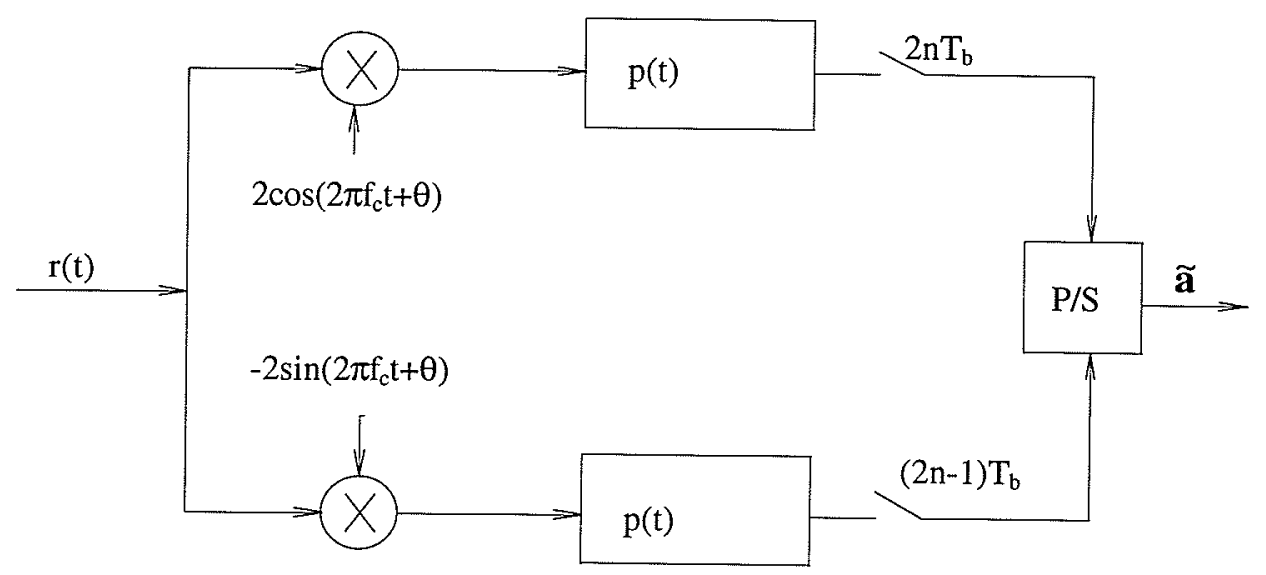

Figure 3-2 Quadrature Correlation filter for MSK modulations

$$
\text { MSK: } p(t)=\frac{1}{T_{b}} \cos \left(\frac{\pi t}{2 T_{b}}\right) \quad-T_{b} \leq t \leq T_{b}
$$

\subsubsection{Performance in AWGN}

The optimum receiver for MSK modulations in AWGN is the quadrature correlation receiver as shown in Figure 3-2. The quadrature correlation receiver performs matched filtering on each quadrature symbol. The symbol decisions in each branch are statistically independent from each other because with reasonable $f_{c}$ the quadrature matched filters are effectively orthogonal to each other. Under the assumption of equally likely independent bits, the probability of bit error $\left(\mathrm{P}_{\mathrm{b}}\right)$ is two times the probability of symbol error $\left(\mathrm{P}_{\mathrm{e}}\right)$ minus the square of the probability of symbol error.

This probability of bit error $\mathrm{P}_{\mathrm{b}}$ of power spectral density No/2 watts/Hz is given by:

where:

$$
\begin{gathered}
\mathrm{P}_{\mathrm{b}} \cong 2 \mathrm{Q}\left(\sqrt{\frac{2 \mathrm{E}_{\mathrm{b}}}{\text { No }}},\right. \\
\mathrm{Q}(\mathrm{x})=\frac{1}{\sqrt{2 \pi}} \int_{\mathrm{x}}^{\mathrm{e}} \exp \left(-\frac{\mathrm{x}^{2}}{2}\right) \mathrm{dx} .
\end{gathered}
$$




\subsubsection{Bandwidth Properties.}

The bandwidth properties are analyzed in terms of the average power density spectrum (PDS) of the modulated signals. According to the Wiener-Khintchine Theorem, if $s(t)$ is wide sense stationary, then the PDS is obtained from the Fourier transform of the autocorrelation function. When the phase of the carrier is assumed unknown and modeled as a uniform random variable, then the modulated signal is considered wide-sense stationary. Typically the modulated signal is viewed from a non coherent standpoint, say an adjacent channel, and hence the wide-sense stationary model is used here. Note if the phase of the carrier is known then the modulated signal is cyclostationary, where the mean and the autocorrelation function are periodic in time.

Since $s(t)$ is a passband process, the PDS of $s(t), S(f)$, is related to the PDS of the complex baseband envelope $\hat{s}(t), S_{c}(f)$, by:

$$
S(f)=\frac{1}{4}\left(S_{c}\left(f-f_{c}\right)+S_{c}\left(-f-f_{c}\right)\right)
$$

For the MSK modulations, the autocorrelation function $\mathrm{R}(\tau)$, which is the expected value of the product $\hat{s}(\mathrm{t})(\mathrm{s}(\mathrm{t}+\tau))^{*}$ is:

$$
R(\tau)=\mathrm{E}_{\mathrm{a}^{\mathrm{I}}, \mathrm{a}}^{\mathrm{Q}}\left(\begin{array}{l}
\left(\mathrm{A} \sum_{\mathrm{k}} \mathrm{a}_{\mathrm{k}}^{\mathrm{i}} \mathrm{p}\left(\mathrm{t}-\mathrm{k} 2 \mathrm{~T}_{\mathrm{b}}\right)-\mathrm{jA} \sum_{\mathrm{k}} \mathrm{a}_{\mathrm{k}}^{\mathrm{Q}} \mathrm{p}\left(\mathrm{t}-\mathrm{k} 2 \mathrm{~T}_{\mathrm{b}}-\mathrm{T}_{\mathrm{b}}\right)\right) . \\
\left(\mathrm{A} \sum_{\mathrm{i}} \mathrm{a}_{\mathrm{k}}^{\mathrm{I}} \mathrm{p}\left(\mathrm{t}-\mathrm{k} 2 \mathrm{~T}_{\mathrm{b}}+\tau\right)+\mathrm{jA} \sum_{\mathrm{k}} \mathrm{a}_{\mathrm{k}}^{\mathrm{Q}} \mathrm{p}\left(\mathrm{t}-\mathrm{k} 2 \mathrm{~T}_{\mathrm{b}}-\mathrm{T}_{\mathrm{b}}+\tau\right)\right)
\end{array}\right)
$$

Since $\mathbf{a}^{\mathrm{I}}$ and $\mathbf{a}^{\mathrm{Q}}$ are sequences of statistically independent (+/-1) random variables, $\mathrm{R}(\tau)$ simplifies to:

$$
R(\tau)=\frac{A^{2}}{T_{b}} \int_{-2 T_{b}}^{2 T_{b}} p(t) p(t+\tau) d t
$$




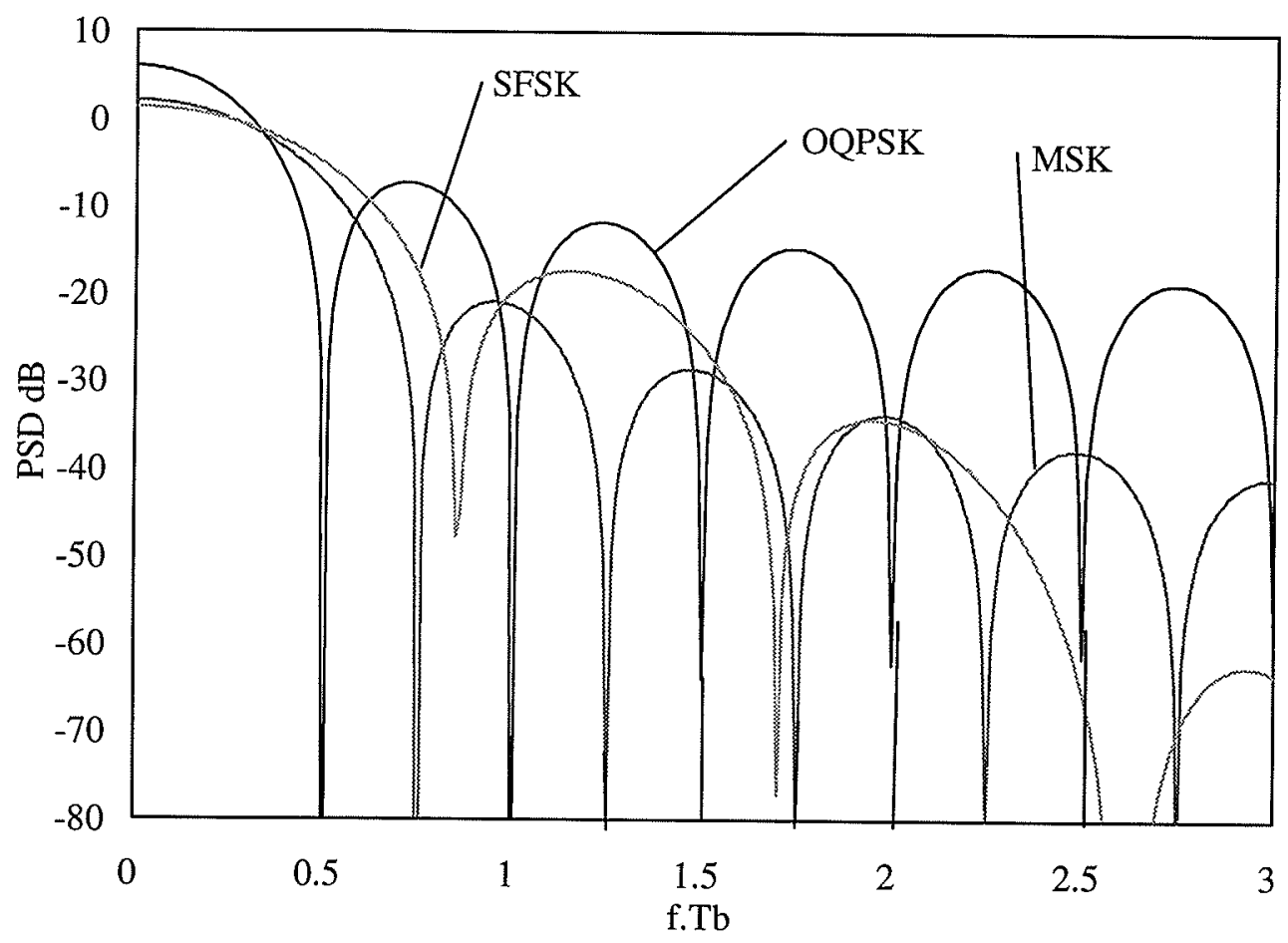

Figure 3-3 PDS of OQPSK, SFSK, and MSK

Therefore, the PDS of the complex envelope $\mathbf{S}_{\mathrm{c}}(\mathrm{f})$ is:

$$
\begin{aligned}
& \quad \mathbf{S}_{c}(\mathbf{f})=\frac{A^{2}}{T_{b}}|P(f)|^{2} \text { watts/Hz } \\
& \text { where } P(f)=\int_{-\infty}^{\infty} p(t) e^{j 2 \pi t t} d t .
\end{aligned}
$$

Hence the spectral properties of the MSK modulation are directly tied to the spectral properties of the pulse shape. Figure 3-3 shows the PDS of the complex envelopes for these three modulations. The asymptotic spectral roll off decays at the rate of $1 / \mathrm{f}^{2 \mathrm{n}+2}$, where $\mathrm{n}$ is the number of times the pulse can be differentiated before the end points become discontinuous. Hence the PDS of OQPSK decays at $1 / \mathrm{f}^{2}$, MSK decays at 
$1 / \mathrm{f}^{4}$, and SFSK decays at $1 / \mathrm{f}^{6}$. Notice that for modulations with sharper spectral decay, there is a corresponding larger main lobe in the PDS due to the increased concentration of signal power in the main lobe. With this brief discussion of the transmitter implementation for MSK class modulations and their properties, the BTR for this class of modulations is presented next.

\subsection{BTR model for MSK modulations}

\subsubsection{Overview}

The received signal $r(t)$ in $A W G N$ is:

$$
r(t)=s(t)+n(t)
$$

where $s(t)=A \sum_{k} a_{k}^{\mathrm{I}} p\left(t-k 2 T_{b}\right) \cos \left(2 \pi f_{c} t\right)+A \sum_{k} a_{k}^{Q} p\left(t-k 2 T_{b}-T_{b}\right) \sin \left(2 \pi f_{c} t\right)$

$$
\mathrm{A}=\sqrt{2 \mathrm{E}_{\mathrm{b}} / \mathrm{T}_{\mathrm{b}}}
$$

and $n(t)$ is white Gaussian noise with two sided spectral density No/2 watts/Hz. In each quadrature arm, the BTR receiver consists of a bandlimiting filter $h(t)$, sampler, and trellis decoder. Each trellis decoder estimates the respective bit stream which are then deinterleaved to form an estimate of the original bit stream. The receiver model is shown in

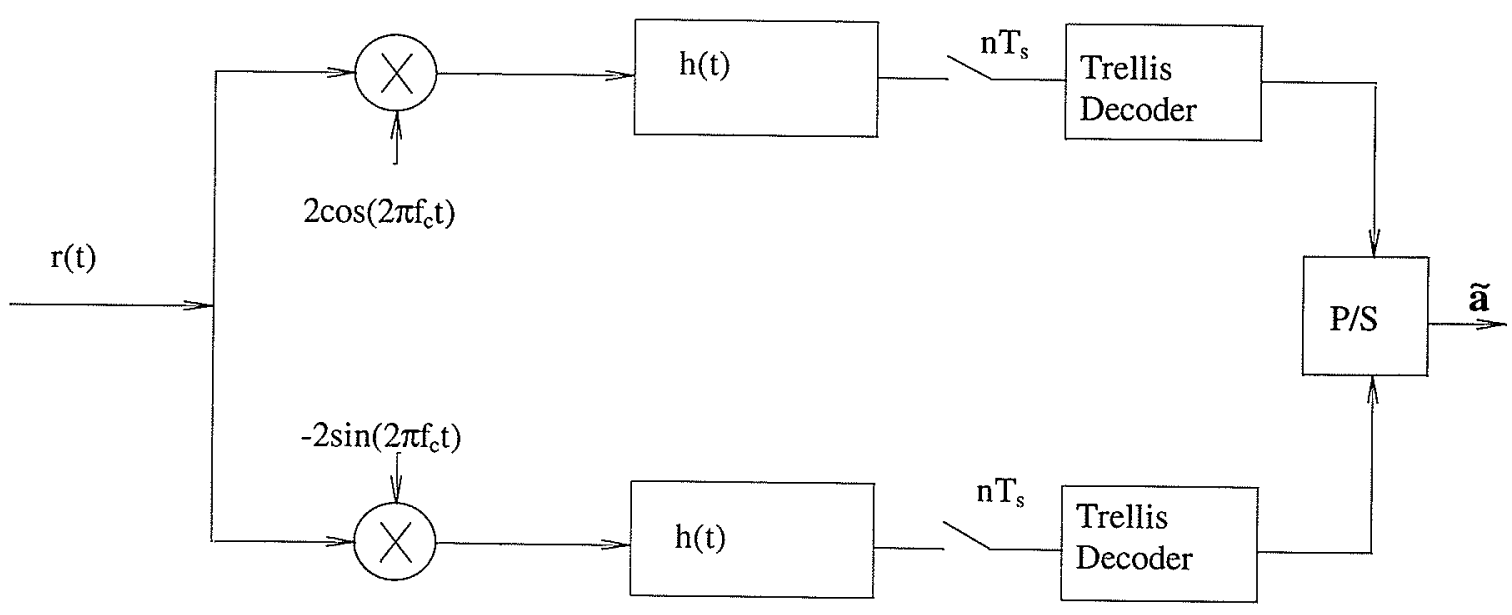

Figure 3-4 BTR receiver for MSK modulations 
Figure 3-4.

\subsubsection{Bandlimiting filter}

The important factors concerning the choice of the bandlimiting filter $h(t)$ are the steepness of the spectral roll off and the effect on the noise statistics. The spectral roll off must be considerably steeper than of the matched filter, otherwise there is no relative benefit in ACI rejection. For ease in implementing the trellis decoding, it is desirable that the samples be very close to being statistically independent. For AWGN, the Power Density Spectrum of the noise at the output of the filter is proportional to $|H(f)|^{2}$. If $|\mathrm{H}(\mathrm{f})|^{2}$ is proportional to a Nyquist form, then Nyquist sampling of the output will result in uncorrelated samples, which because they are Gaussian random variables will be statistically independent. In consideration of these factors, the following filter was chosen:

$$
\begin{aligned}
\mathrm{h}(\mathrm{t}) & =\sin \left(2 \pi \mathrm{W}\left(\mathrm{t}-7.5 \mathrm{~T}_{\text {sy }}\right)\right) /\left(\pi\left(\mathrm{t}-7.5 \mathrm{~T}_{\text {sy }}\right)\right) \quad 0 \leq \mathrm{t} \leq 15 \mathrm{~T}_{\text {sy }} \\
& =0 \text { elsewhere }
\end{aligned}
$$

The bandlimiting filter $\mathrm{h}(\mathrm{t})$ approximates the ideal brick wall filter with impulse response $\sin (2 \pi \mathrm{Wt}) / \pi \mathrm{t}$ with bandwidth $\mathrm{W}$, but truncated to $15 \mathrm{~T}_{\text {sy }}$, with a delay of 7.5 $\mathrm{T}_{\text {sy }}$ to make it causal. Two bandwidths are considered, $\mathrm{W}=0.5 / \mathrm{T}_{\mathrm{b}}$ and $\mathrm{W}=0.25 / \mathrm{T}_{\mathrm{b}}$. Note that $T_{s y}=2 T_{b}$ because of the quadrature modulation. The two chosen bandwidths have Nyquist sampling rates which are commensurate with $\mathrm{T}_{\mathrm{sy}}$. Although the sampling rate need not be commensurate with $\mathrm{T}_{\text {sy }}$ for implementation, this feature makes synchronization as well as the trellis decoding easier to perform. Note that $\mathrm{W}=0.5 / \mathrm{T}_{\mathrm{b}}$ corresponds to the 


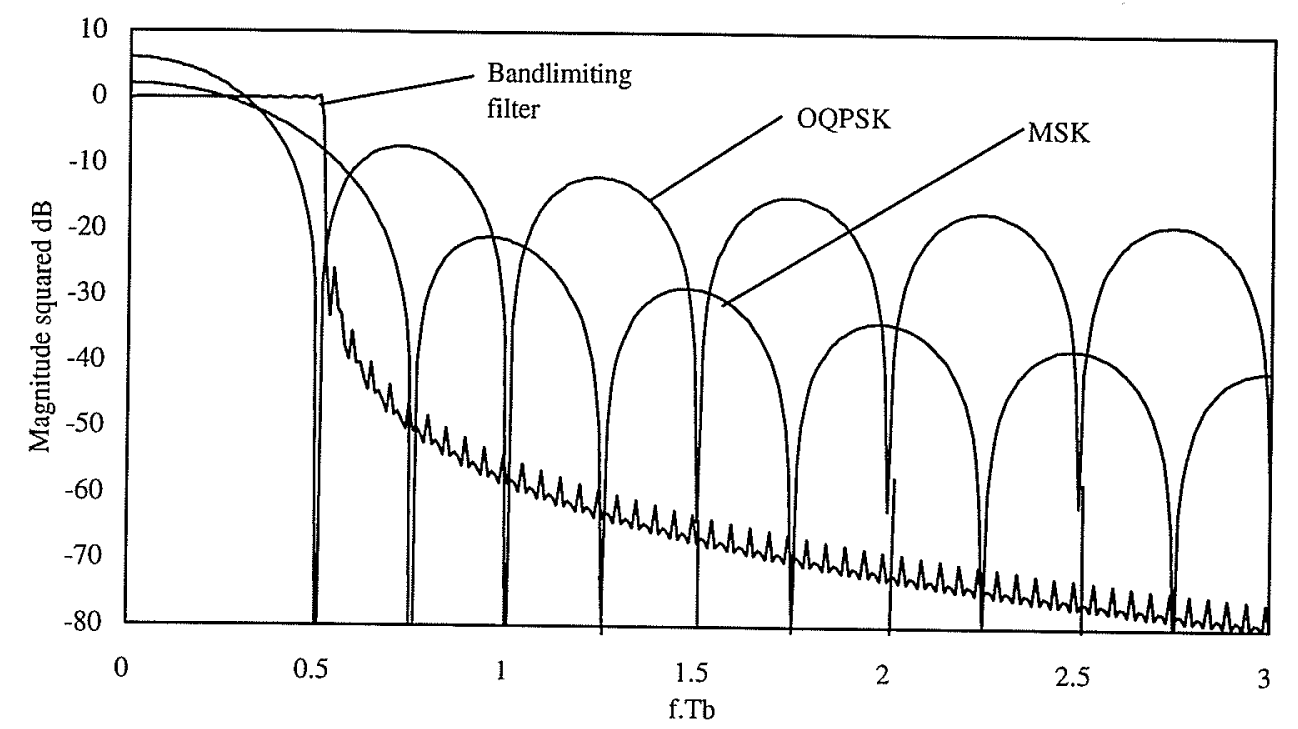

\section{Figure 3-5 Magnitude squared spectral responses for the bandlimiting filter with $\mathrm{W}=0.5 / \mathrm{T}_{\mathrm{b}}$, and for the matched filters of OQPSK and MSK}

$97 \%$ in band power bandwidth for MSK, where as $\mathrm{W}=0.25 / \mathrm{T}_{\mathrm{b}}$ corresponds to $69 \%$.

In order to check that the filter has a narrower spectral roll off then the matched filters, Figure 3-5 shows the magnitude squared spectral response of the matched filters for OQPSK and MSK compared to $|H(f)|^{2}$. Figure 3-5 shows that indeed $h(t)$ with $\mathrm{W}=0.5 / \mathrm{T}_{\mathrm{b}}$ has a steeper roll off than the matched filter. For the case with $\mathrm{W}=0.25 / \mathrm{T}_{\mathrm{b}}, \mathrm{h}(\mathrm{t})$ has the same roll off as indicated by Figure 3-5 but the main lobe is narrower. One way to quantify the difference between the ideal bandlimited filter and the approximation is to compare the amount of power due to an adjacent signal that each filter passes. For one adjacent MSK signal frequency spaced by $2 / T_{b}$ with $W=0.5 / T_{b}$, the filter allows $0.006 \mathrm{~dB}$ more adjacent channel interference (ACI) power than an ideal bandlimited filter. Note for this spacing that the matched filter allows $2.57 \mathrm{~dB}$ more ACI power than the ideal filter.

The correlation between noise samples due to the AWGN at the output of $h(t)$ is 


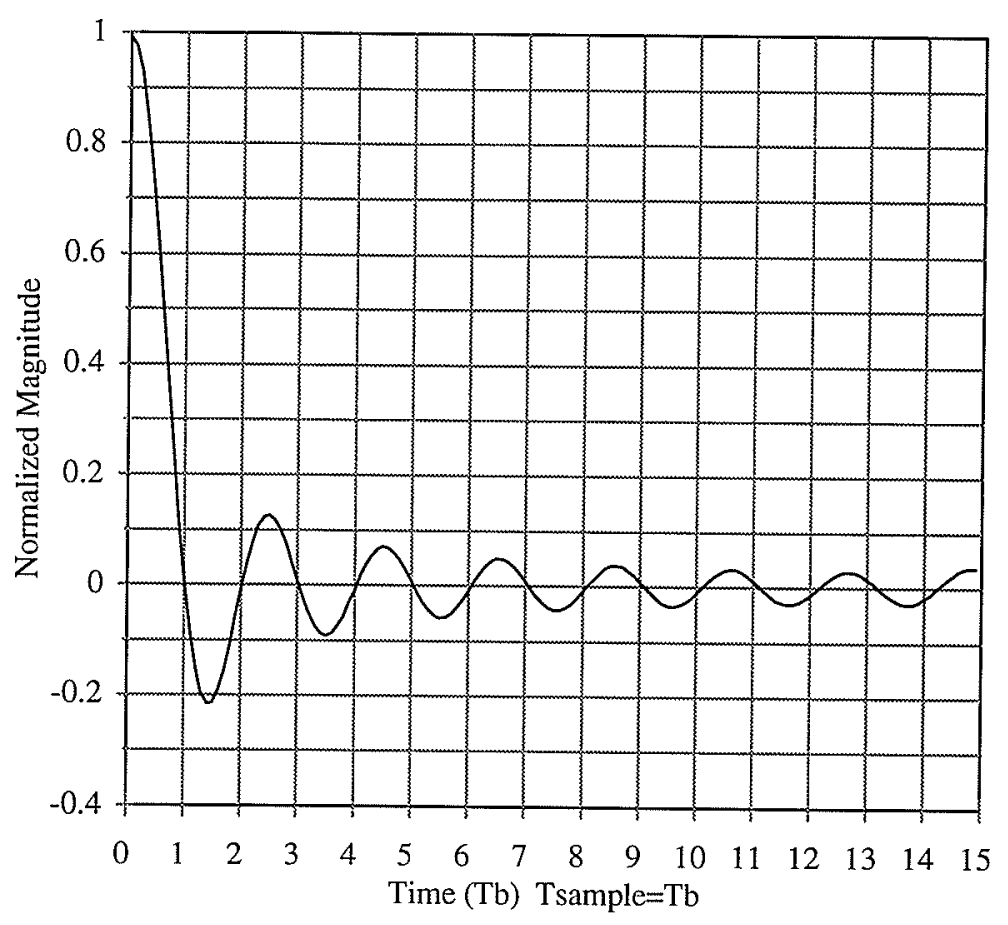

\section{Figure 3-6 Normalized filter autocorrelation function with $\mathrm{W}=0.5 / \mathbf{T}_{\mathrm{b}}$}

determined by the autocorrelation function $\mathrm{R}_{h}(\tau)$ :

$$
R_{h}(\tau)=\int h(t) h(t+\tau) d t
$$

Figure 3.6 shows the normalized autocorrelation of $h(t)$ with $W=0.5 / T_{b}$. For $\mathrm{W}=0.25 / \mathrm{T}_{\mathrm{b}}$, the autocorrelation function has same form except it is scaled by $2 \mathrm{~T}_{\mathrm{b}}$. The figure shows that correlation values between Nyquist sampling times $\left(n / T_{s} n \neq 0\right)$ are approximately zero and can in effect be considered uncorrelated. Since the noise samples are Gaussian, these samples can be considered statistically independent.

The structure of the ISI at the output of the filter with the two bandwidths due to 


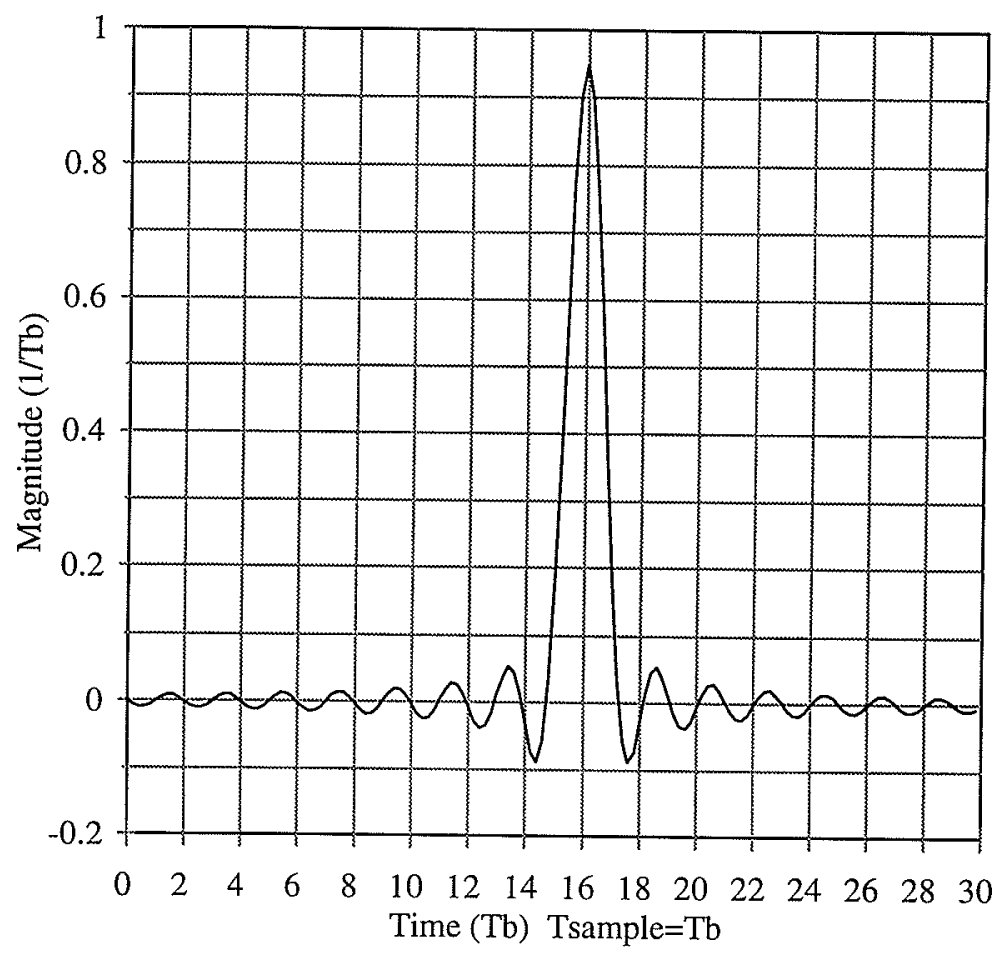

Figure 3-7 Output of $h(t)$ with $W=0.5 / T b$ due to MSK pulse

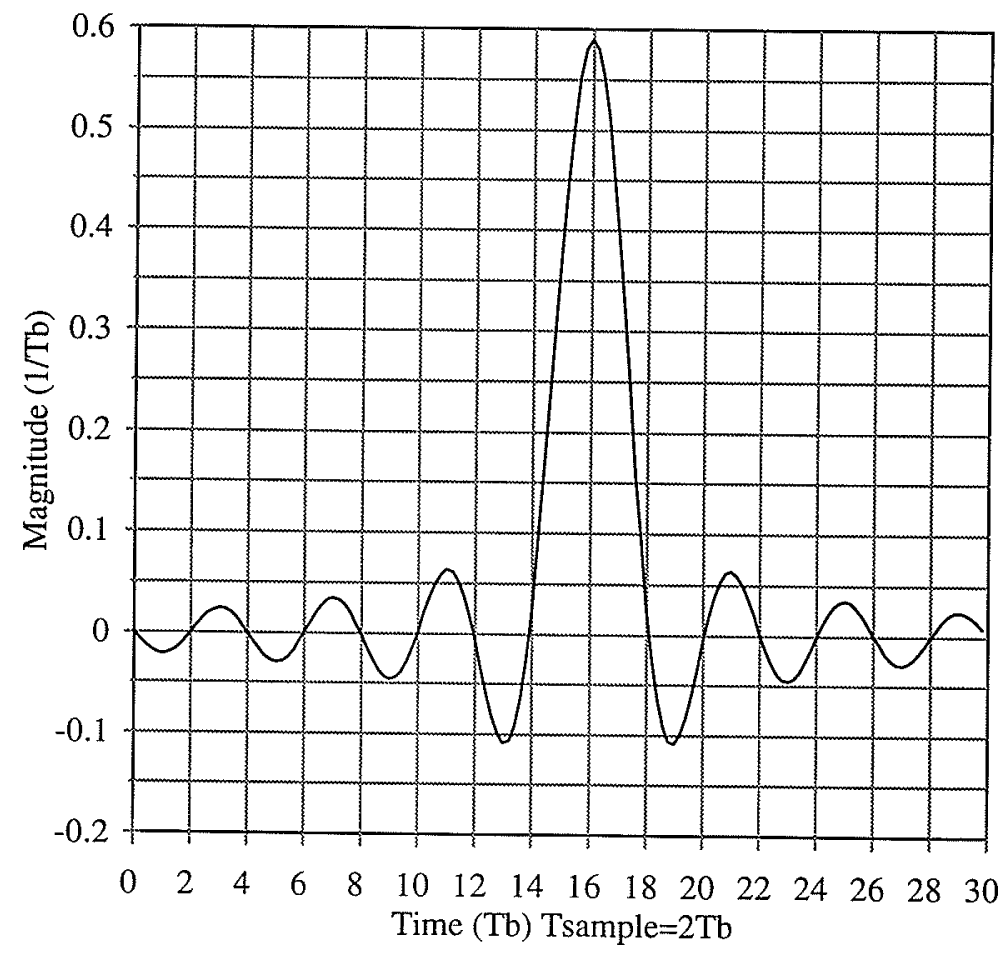

Figure 3-8 Output of $h(t)$ with $W=0.25 / T b$ due to MSK pulse 


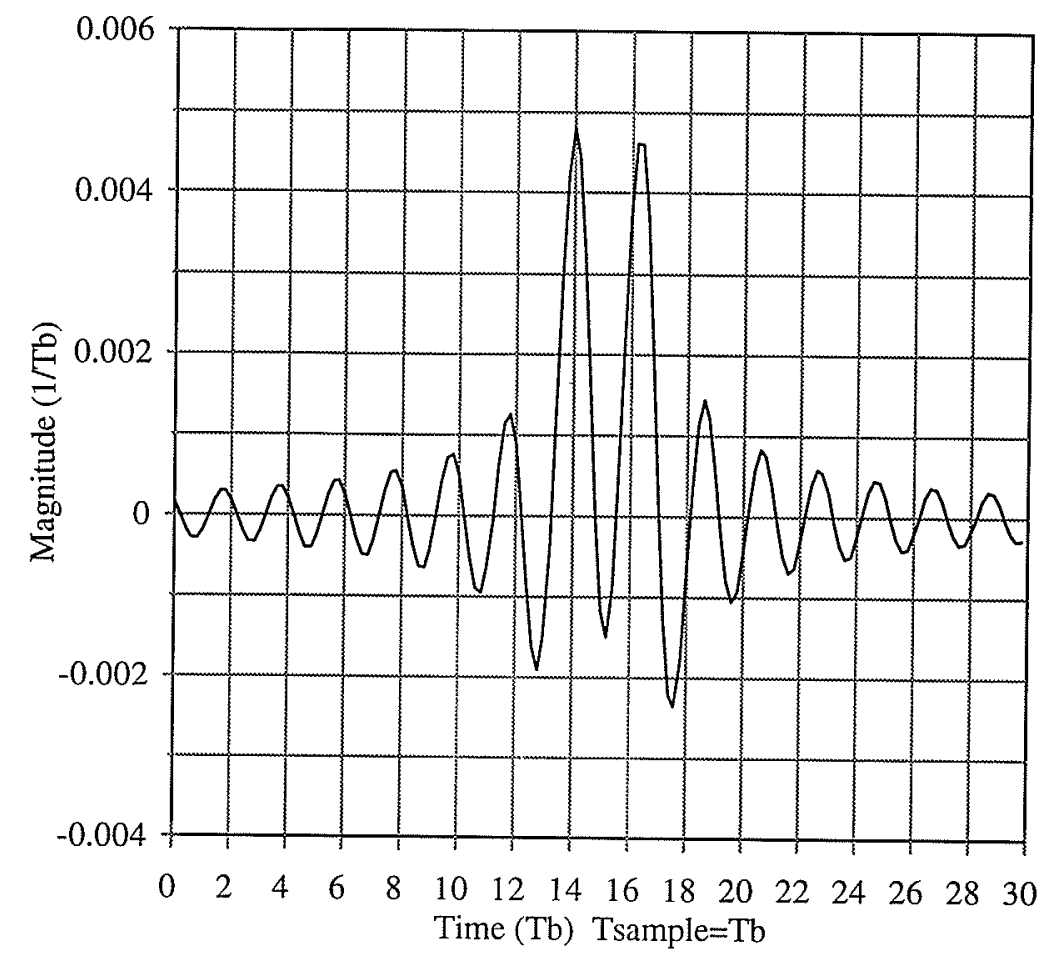

Figure 3-9 Output of $h(t)$ with $W=0.5 / T b$ due to adjacent MSK pulse frequency offset by $2 / \mathrm{Tb}$

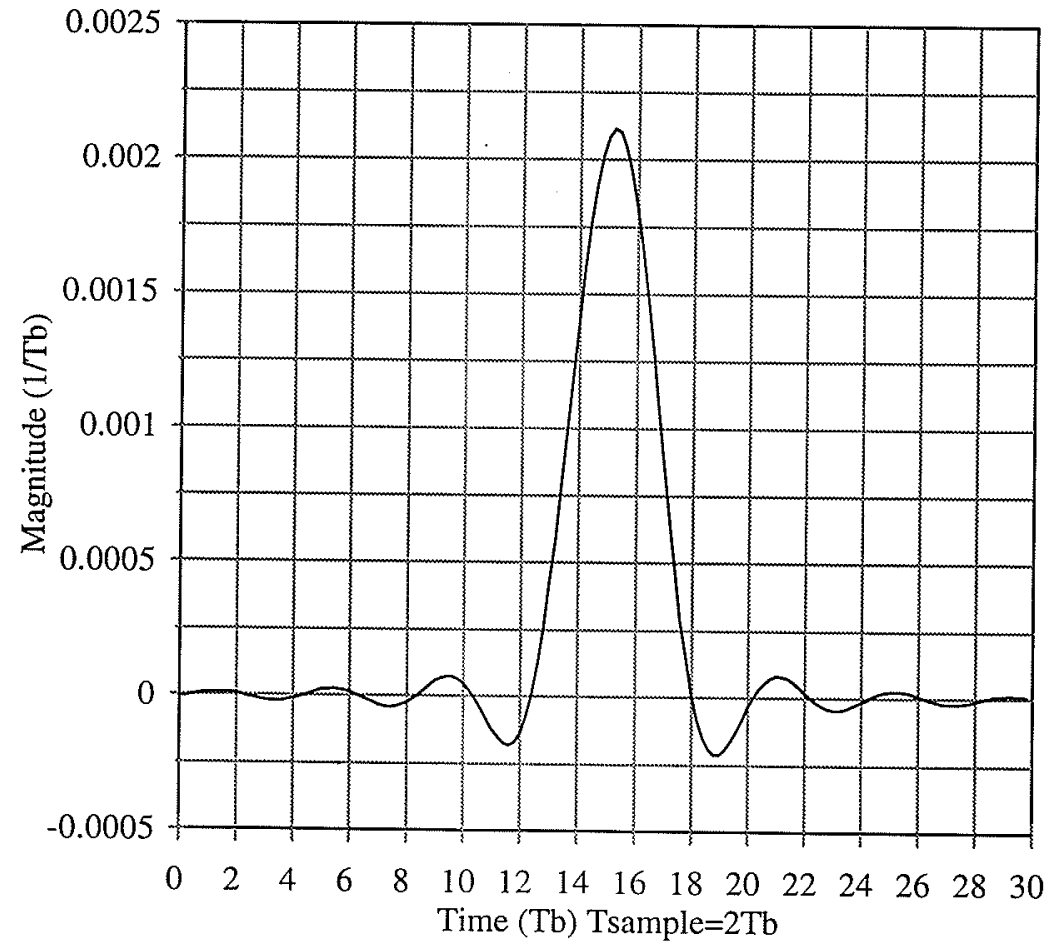

Figure 3-10 Output of $h(t)$ with $W=0.25 / T b$ due to adjacent MSK pulse frequency offset by $2 / \mathbf{T b}$ 
one MSK pulse is shown in Figures 3-7 and 3-8. The samples at the Nyquist sampling times, except for two symbol intervals from 14-18 $\mathrm{T}_{\mathrm{b}}$, are approximately zero for the two cases. Although the filter's impulse has length of $15 \mathrm{~T}_{\text {sy }}$, there is only ISI for one symbol length when Nyquist sampled. One explanation is that the filtered signals spectrum is approximately flat over the limited bandwidth, particularly for $\mathrm{W}=0.25 / \mathrm{T}_{\mathrm{b}}$, which thus approximates the flat bandlimited spectrum of a Nyquist pulse. From an energy standpoint point, the samples from these two symbol intervals represent over $99.99 \%$ of the total energy of the filtered signal for all the modulations considered.

The structure of a filtered adjacent signal pulse is contrasted to the filtered signal of interest ( $\mathrm{SOI}$ ) pulse. Figure 3-9 shows the output of $h(t)$ with $W=0.5 / T_{b}$ due to one adjacent MSK pulse with a frequency offset by $2 / T_{b}$. This filtered pulse's energy is more spread out over the time axis, which gives rise to more significant ACI terms. The structure of this filtered pulse only depends on the bandwidth of the filter, since the synchronization offset simply shifts this filtered pulse in time, where as the phase and frequency offset modify the magnitude of this filtered pulse. Figure 3-10 shows the output of $h(t)$ with $W=0.25 / T_{b}$ due to one adjacent MSK pulse with a frequency offset of $2 / T_{b}$. Here the filtered output is a pulse, approximately half cosine in shape, which extends over 3 symbol lengths. Note that the magnitude of this pulse is dependent on the random phase offset parameter. The trend with even narrower filtering is to elongate this pulse over the time axis. Again this filtered pulse has more significant ACI terms than for the corresponding filtered SOI pulse. 


\subsubsection{Sufficient Statistics}

Assuming that the filtered signal is ideally bandlimited to $\mathrm{W}$, then the signal can be uniquely represented by a linear combination of the orthogonal basis set for W bandlimited space, which is $\{\sin (2 \pi \mathrm{W}(\mathrm{t}-\mathrm{n} / 2 \mathrm{~W})) / \pi(\mathrm{t}-\mathrm{n} / 2 \mathrm{~W})\}$ where $\mathrm{n} \in \mathrm{Z}$. These basis functions have the property that at $t=n / 2 W$ all other basis functions equal zero except the function which has been translated by $\mathrm{n} / 2 \mathrm{~W}$ from the origin. Hence sampling at $2 \mathrm{~W}$, the Nyquist rate, provides sufficient statistics to the trellis decoder, since the original bandlimited signal can be reconstructed from these samples.

Since the samples are the coefficients of orthogonal bases set, the Euclidean distance between two filtered signals, say $s_{1}(t)$ and $s_{2}(t)$, can be computed in terms of their samples $\left(\mathrm{s}_{1 \mathrm{i}}\right\}$ and $\left\{\mathrm{s}_{2 \mathrm{i}}\right\}$.Thus,

$$
\left\|s_{1}(t)-s_{2}(t)\right\|^{2}=\frac{1}{2 W} \sum_{i}\left(s_{1 i}-s_{2 i}\right)^{2}
$$

\subsection{Trellis Decoder.}

\subsubsection{Trellis Description in AWGN}

Trellis description of ISI follows when the ISI resulting from the causal bandlimiting filter $h(t)$ is modeled as a finite state machine. To develop the trellis consider first the sampled vector $\mathbf{z}^{\mathrm{I}}$ of the inphase filter ouput the $\mathrm{j}^{\text {th }}$ symbol interval:

$$
\mathbf{z}_{j}^{I}=\sum_{k=0}^{L} a_{j-k}^{I} \mathbf{g}_{k}+\mathbf{n}_{j}^{I}
$$

where $g_{k, i}=g\left(t=k T_{s y}+i T_{s}\right)=\int_{-\infty}^{o} p\left(t-\tau-T_{b}\right) h(\tau) d \tau, L$ is the memory length in units of $\mathrm{T}_{\mathrm{sy}}$ of the filter, $\mathbf{n}_{\mathbf{j}}^{\mathbf{I}}$ is the sampled noise of variance $2 \mathrm{NoW}$ watts, and $\left\{\mathbf{g}_{\mathrm{k}}\right\}$ is the sampled impulse response of the overall channel. Note that a state is defined over $\mathrm{T}_{\mathrm{sy}}$ not $\mathrm{T}_{\mathrm{S}}$, and hence the channel coefficients $\left\{\mathrm{g}_{\mathrm{i}}\right\}$, noise input $\mathbf{n}_{\mathbf{j}}$, and output $\mathbf{z}_{\mathrm{i}}$ are [1xS] vectors where $S$ is equal to $T_{\text {sy }} / T_{s}$. The memory vector of the channel defines the state and 


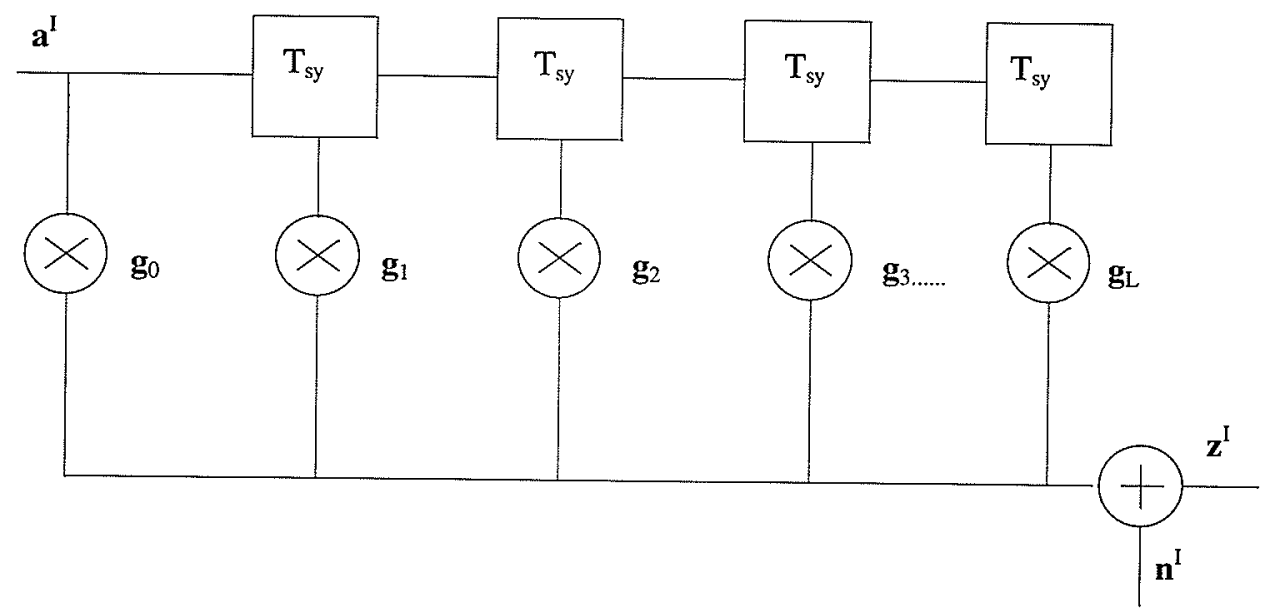

Figure 3-11 Finite state machine model of intersymbol interference

the number of states equals $2^{\mathrm{L}}$. The state is defined for the whole symbol length $\mathrm{T}_{\mathrm{sy}}$ because the transitions of this finite state machine occur every $\mathrm{T}_{\mathrm{sy}}$. Figure 3-11 shows the finite state machine model of the causal bandlimiting filter.

A trellis comprises of defining the mappings from an information vector $\mathbf{a}^{\mathbf{I}}$ of length $\mathrm{L}$ to a set of discrete output vectors $\left\{\mathrm{s}_{\mathrm{i}}\right\}$, as well as the allowable transitions between the states which are defined by $L$ information symbols. In this case, the finite state machine model of the ISI provides both the set of mappings and allowable transitions sufficient for a trellis description. The trellis is shown in Figure 3-12, where the memory length is 2 .

\subsubsection{Decoding the Trellis in AWGN}

The maximum likelihood sequence estimation (MLSE) method for estimating the information sequence (vector) $\mathbf{a}^{\mathbf{I}}$ given the received vector $\mathbf{z}^{\mathbf{I}}$ is to choose the $\mathbf{a}^{\mathbf{I}}$ that maximizes the conditional probability $\mathrm{P}\left\{\mathbf{z}^{\mathrm{I}} \mid \mathbf{a}^{\mathrm{I}}\right\}$. In order to evaluate $\mathrm{P}\left\{\mathbf{z}^{\mathrm{I}} \mid \mathbf{a}^{\mathrm{I}}\right\}$, the statistics of noise vector $\mathbf{n}^{\mathrm{I}}$ are required. For the case of AWGN, the statistics of $\mathbf{n}^{\mathrm{I}}$ are known. They are identical independent Gaussian random variables with zero mean and a variance of $2 \mathrm{NoW}$ watts. For a block length Ns, the MLSE rule chooses $\mathbf{a}^{\mathbf{I}}$ that maximizes the total metric: 


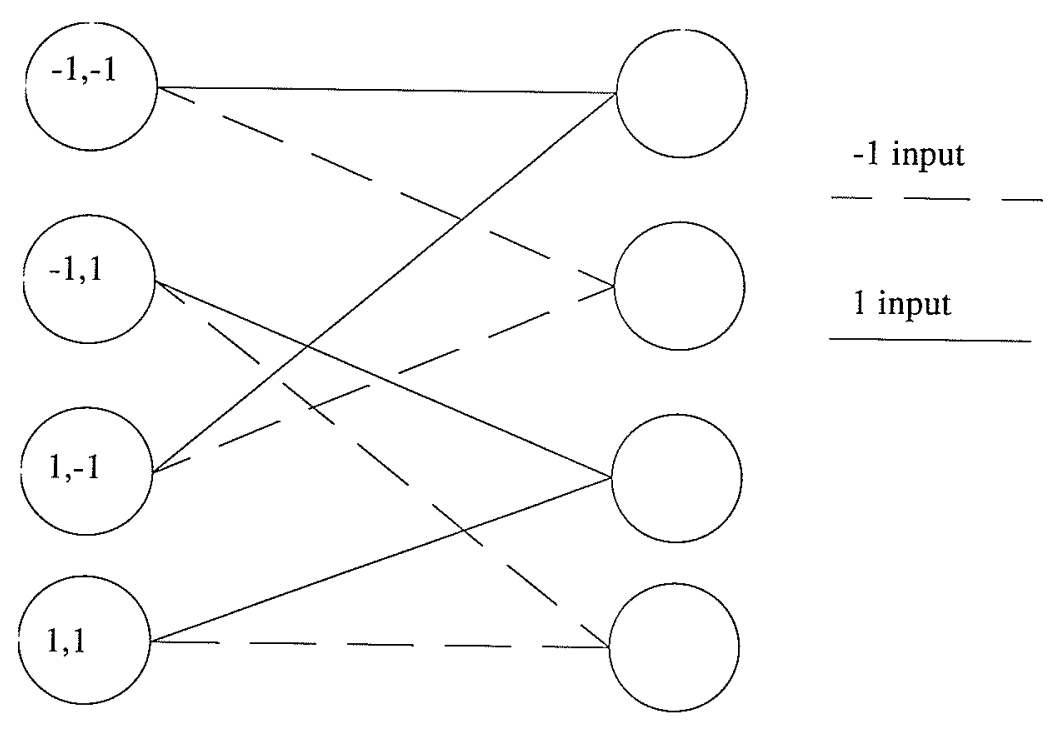

Figure 3-12 Trellis with $\mathrm{L}=2$

$$
\ln \left[P\left\{\mathbf{z}^{I} \mid \mathbf{a}^{\mathrm{I}}\right\}\right]=-\sum_{\mathrm{j}=1}^{N s}\left\|\mathrm{z}_{\mathrm{j}}^{\mathrm{I}}-\sum_{\mathrm{k}=0}^{\mathrm{L}} \mathrm{a}_{\mathrm{j}-\mathrm{k}}^{\mathrm{I}} \mathbf{g}_{\mathrm{k}}\right\|^{2}
$$

Note that $\mathbf{z}_{\mathbf{j}}^{\mathbf{I}}$ and $\mathbf{g}_{\mathrm{k}}$ are $1 \mathrm{xS}$ vectors, and the metric in (3.14) is defined over $\Re^{\mathrm{s}}$. The sum in equation (3.14) is the total metric for a given sequence $\mathbf{a}^{\mathbf{x}}$. The individual terms due to each output transition in the total metric are called branch metrics. At each transition there are only $2^{\mathrm{L}}$ possible branch metrics, since the information symbols are binary. Without any simplification, to compute the total metric for all possible sequences would require a prohibitive $2^{\mathrm{Ns}}$ computations. The Viterbi Algorithm, however, dramatically reduces the number of computations by exploiting the trellis description to compute the total metric.

\subsubsection{The Viterbi Algorithm}

There is a wealth of literature on the VA, particularly as a means to implement MLSE for linear ISI channels[19] . A brief discussion of the VA follows, in order to 
highlight the basic assumptions by which the VA works. The VA searches the trellis for the most likely path according to the following steps.

1) The VA keeps one survivor path at each state, while retaining the accumulated metric of that path. Hence there are $2^{\mathrm{L}}$ survivor paths kept at each iteration..

2) For the next iteration, the VA extends these $2^{\mathrm{L}}$ survivor paths to $2^{\mathrm{L}+1}$ paths and computes the new accumulated metrics of these $2^{L+1}$ paths by adding the new branch metric to each.

3) At each state, the VA then rejects the path with the smaller metric, and hence retains $2^{\mathrm{L}}$ new survivor paths with their new accumulated metrics.

The above is repeated until end of block is reached. The survivor path with the largest accumulated metric is then chosen

Note that the VA requires $\mathrm{Ns} 2^{\mathrm{L}}$ computations compared to the brute force $2^{\mathrm{Ns}}$. The key idea of the VA is that if two paths merge in a trellis state, then the path with the smaller metric can be rejected because any two signals which have the same signal component after the merge, the signal whose 'tail' has a larger metric will always be larger. The main assumption behind this idea is that future metrics are independent of present and past metrics. More generally, future received samples are statistically independent of present and past received samples, and hence knowledge of future samples does not change the likelihood of present and past samples. In the case of AWGN and the causal bandlimiting filter with $[\mathrm{H}(\mathrm{f})]$ very close to Nyquist 1 form, the received samples are statistically independent and thus the VA effectively performs MLSE.

\subsubsection{Truncated ISI}

Recall that for the chosen filter the total ISI spans 15 symbol lengths, with over $99.9 \%$ of the total energy contained in three symbol lengths. The trellis decoder ignores 
all the other ISI terms except from the 3 symbol lengths mentioned, which gives the trellis a memory length of two ( $\mathrm{L}=2$ ). The residual ISI is an amplitude bounded random variable which is below $-30 \mathrm{~dB}$ power relative to the signal power; hence it has minimal to no effect on performance.

\subsection{Trellis Decoder In AWGN and ACI}

In order to understand the impact of the filtered ACI in the decoding of the ISI trellis of the signal of interest (SOI), it is necessary to examine the structure of this ACI to estimate its first and second order statistics. In the presence of $\mathrm{ACI}$, the received signal at the front end of the receiver is:

$$
\text { where } \quad \begin{aligned}
r(t) & =\operatorname{As}(t)+a(t)+n(t) \\
a(t) & =\sum_{i=1}^{N a} \sum_{j} A_{i} a_{i, j}^{1} p_{i}\left(t-j 2 T_{b}-\lambda_{i}\right) \cos \left(2 \pi f_{i} t+\theta_{i}\right) \\
& -\sum_{i=1}^{N a} \sum_{j} A_{i} a_{i, j}^{Q} p_{i}\left(t-j 2 T_{b}-T_{b}-\lambda_{i}\right) \sin \left(2 \pi f_{i} t+\theta_{i}\right)
\end{aligned}
$$

is the ACI signal, $\mathrm{Na}$ is the number of interferers, $\left\{\mathrm{A}_{\mathrm{i}}, \mathrm{f}_{\mathrm{i}}, \lambda_{\mathrm{i}}, \theta_{\mathrm{i}}\right\}$ are the parameters of each interferer, and $\lambda_{i}, \theta_{i}$ are independent uniform random variables over $(0,2 \pi),\left(-T_{b}, T_{b}\right)$ respectively. It is assumed that all the inphase and quadrature bit streams of the interferers are independent equally likely random variables. For the inphase trellis decoder, then the sampled signal for the $\mathrm{j}^{\prime}$ th symbol time is:

$$
\begin{aligned}
& \mathbf{z}_{\mathrm{j}}=\mathbf{s}_{\mathrm{j}}+\sum_{\mathrm{i}=1}^{\mathrm{Na}} \mathbf{A} \mathbf{I}_{\mathrm{i}, \mathrm{j}}-\sum_{\mathrm{i}=1}^{\mathrm{Na}} \mathbf{A} \mathbf{Q}_{\mathrm{i}, \mathrm{j}}+\mathbf{n}_{\mathrm{j}} \\
& \mathbf{A I}_{\mathrm{i}, \mathrm{j}}=\mathrm{A}_{\mathrm{i}} \sum_{\mathrm{k}=1}^{\mathrm{N}} \mathrm{a}_{\mathrm{i}, \mathrm{j}-\mathrm{k}}^{\mathrm{I}} \mathbf{g}_{\mathrm{i}, \mathrm{k}}^{\mathrm{I}}, \mathbf{A Q _ { i , j }}=\mathrm{A}_{\mathrm{i}} \sum_{\mathrm{k}=1}^{\mathrm{N}} \mathrm{a}_{\mathrm{i}, \mathrm{j}-\mathrm{k}}^{\mathrm{Q}} \mathbf{g}_{\mathrm{i}, \mathrm{k}}^{\mathrm{Q}} \\
& g_{i, j}^{I}=g^{I}\left(t=i T_{s y}+j T_{s}\right)=\int_{-\infty}^{\infty} p\left(\tau-T_{b}-\lambda_{i}\right) \cos \left(2 \pi\left(f_{i}-f_{c}\right) \tau+\theta_{i}\right) h(t-\tau) d \tau \\
& g_{i, j}^{Q}=g^{Q}\left(t=i T_{s y}+j T_{s}-T_{b}\right)=\int_{-\infty}^{o} p\left(\tau-T_{b}-\lambda_{i}\right) \sin \left(2 \pi\left(f_{i}-f_{c}\right) \tau+\theta_{i}\right) h(t-\tau) d \tau
\end{aligned}
$$

where $\mathbf{A I}_{i, j}, \mathbf{A Q}_{\mathrm{i}, \mathrm{j}}$ are $1 \mathrm{XS}$ vectors of the inphase and quadrature interference due to the $i^{\prime}$ th interferer. 


\subsubsection{First Order Statistics}

Since the filtered adjacent signal is time dispersed, the sample value due to the filtered $\mathrm{ACI}$ is a sum of independent equally likely random variables each multiplied by a coefficient of approximately the same magnitude. For one interferer, there are two sets of terms due to the inphase and quadrature interference. For the case with $\mathrm{W}=0.5 / \mathrm{T}_{\mathrm{b}}$, there are approximately 30 terms, and with $\mathrm{W}=0.25 / \mathrm{T}_{\mathrm{b}}$, there are approximately 6 terms, as previously indicated for one interferer. By invoking the central limit theorem, it is a reasonable assumption that the sampled filtered $\mathrm{ACI}$ in both cases, but particularly with $\mathrm{W}=0.5 / \mathrm{T}_{\mathrm{b}}$, tends to a first order density Gaussian random variable. Note this assumption is even truer in the presence of more than one interferer of the same relative power.

One way to check the accuracy of this Gaussian approximation is to estimate the first order density function by a Monte Carlo simulation. The two examples examined are one MSK adjacent signal with a frequency offset by $2 / T_{b}$ and filtered with $W=0.5 / T_{b}$ and $\mathrm{W}=0.25 / \mathrm{T}_{\mathrm{b}}$.

The estimate of the density function is based on the histogram method. The range of the samples is divided into equal sized bins, and the estimate of the probability at the center of a bin is simply the number of samples falling in that bin divided by the product of the total number of samples and the bin size. The number of bins used here is 200 .

The density estimate of the filtered ACI with $\mathrm{W}=0.5 / \mathrm{T}_{\mathrm{b}}$ in Figure 3-13 is shown to be very close to a Gaussian density with the same variance, which verifies in this case that the Gaussian approximation is reasonable. To check the robustness of this assumption, the same Monte Carlo density estimate of the filtered ACI was run for the case of one interferer, with frequency offsets of $2.0 / \mathrm{T}_{\mathrm{b}}, 1.5 / \mathrm{T}_{\mathrm{b}}, 1.0 / \mathrm{T}_{\mathrm{b}}$. The results showed the density estimate had the same form for all cases, which is expected since the frequency spacing only modifies the magnitude of the filtered adjacent signal pulse and does not change its form. The density estimate for $W=0.25 / T_{b}$ in Figure $3-14$ only 


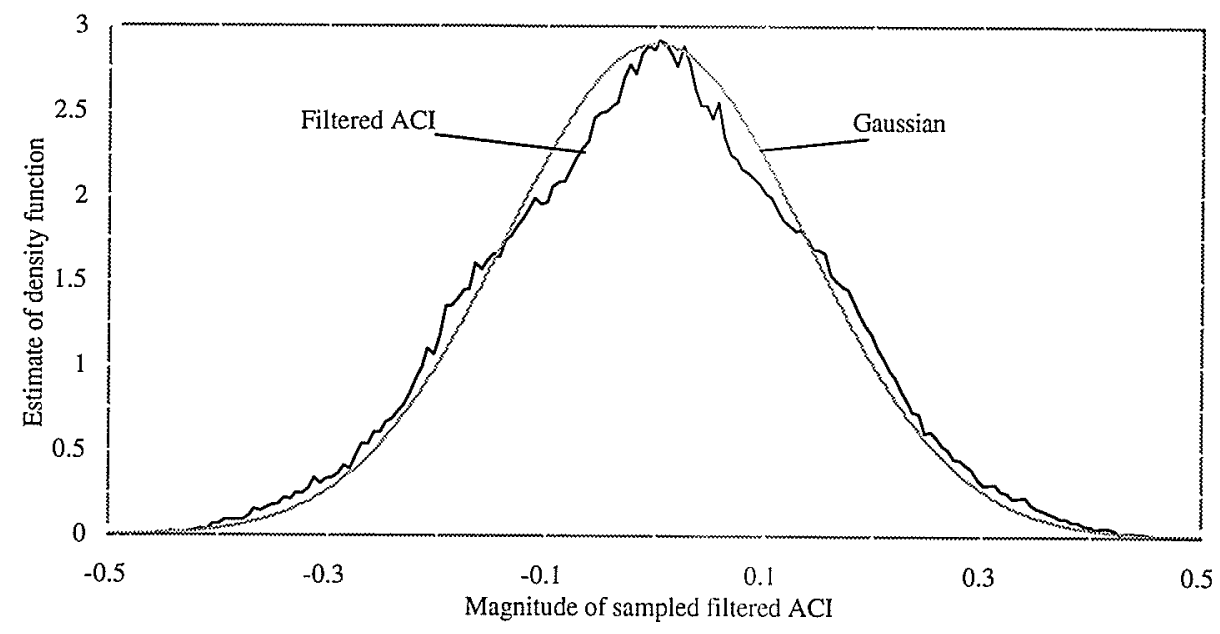

Figure 3-13 Estimate of amplitude density of filtered MSK pulse frequency offset at $2.0 / \mathrm{Tb}$ with $\mathrm{W}=0.5 / \mathrm{Tb}$ at $23 \mathrm{~dB}$ interference power to signal power, and Gaussian density with same variance as filtered ACI.

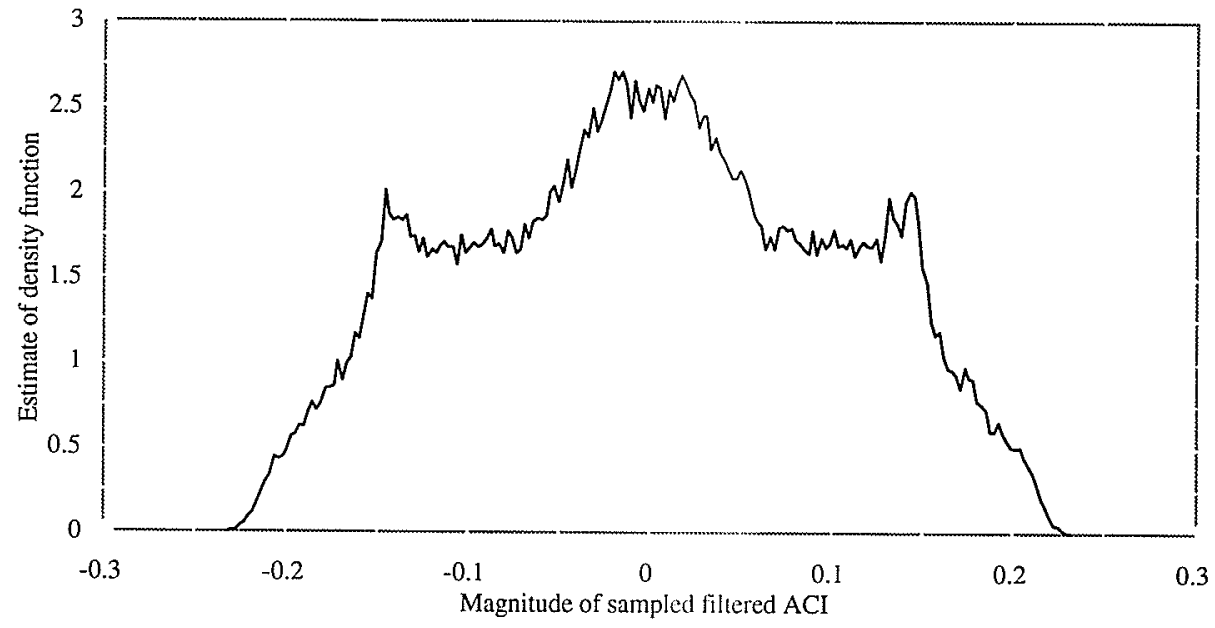

Figure 3-14 Estimate of amplitude density of filtered adjacent MSK pulse frequency offset at $2.0 / \mathrm{Tb}$ with $\mathrm{W}=0.25 / \mathrm{Tb}$ at $23 \mathrm{~dB}$ interference power to signal power 
approaches the form of a Gaussian density, since there are significantly less terms of the filtered adjacent signal pulse.

\subsubsection{Second Order Statistics.}

The approach taken here is to model the filtered ACI as white Gaussian noise. The advantage of this approach is that the receiver does not require knowledge of any parameter of the ACI. The accuracy in this model of filtered ACI as additive white Gaussian noise is examined later in the simulations. There may be benefit, however, in modeling the filtered $\mathrm{ACI}$ as colored Gaussian noise, where this interference spectrum is given by the power density spectrum of the filtered ACI. In order to whiten this interference noise, however, the receiver needs to know the relative power level of the AWGN and the filtered ACI. In addition, there may be some benefit in exploiting the statistical dependence between quadrature branches which exist because of the ACI. The trellis decoder thus performs a sub optimum MLSE by modeling the filtered ACI as AWGN, hence requiring no changes in its metrics from the case of no ACI.

\subsection{Summary}

The MSK class modulation has been described as a class of constant envelope linear offset quadrature modulation. The matched filter is shown as optimum in the presence of AWGN only. The BTR was developed for the MSK class modulations, where

the received signal is projected onto a bandlimited subspace by the filter followed by Nyquist sampler, and then processed by a trellis decoder. The BTR performs a MLSE on the bandlimited subspace in AWGN, and performs a sub optimum MLSE in the presence of ACI by modeling the filtered ACI as AWGN. The next chapter investigates the performance both analytically and with simulations of the BTR compared with the matched filter in AWGN and in ACI. 


\section{BTR PERFORMANCE IN AWGN AND ACI}

The performance of the BTR in the cases of AWGN and with ACI are investigated both analytically and by simulation. This performance shows that the BTR has comparable performance with the optimum matched filter (MF) in the case of AWGN. In addition, the BTR is compared with a simpler receiver (SR) with no trellis decoder which samples the filtered signal every symbol interval. The BTR is shown to have a performance gain relative to the SR by exploiting the energy of the samples not detected by the SR as well as avoiding degradation due to any residual ISI. For the case of ACI, the BTR has significant performance gain up of to $4.8 \mathrm{~dB}$ compared to the MF. The programs for the simulations were written in $\mathrm{C}$ and were executed on a Sun workstation.

\subsection{BTR PERFORMANCE IN AWGN}

The performance of the BTR compared to the MF in AWGN is investigated both analytically and with simulations for OQPSK, MSK and SFSK. The analysis indicates that for these modulations there is negligible degradation in minimum distance for the BTR with $\mathrm{W}=0.5 / \mathrm{T}_{\mathrm{b}}$, but with $\mathrm{W}=0.25 / \mathrm{T}_{\mathrm{b}}$ there is from $1.11 \mathrm{~dB}$ to $2.15 \mathrm{~dB}$ degradation depending on the modulation. Monte Carlo simulation which exactly model the channel conditions verify the results of the analysis.

\subsubsection{Error Analysis}

For moderate to high SNR, the error performance of the trellis decoder is determined by the minimum Euclidean distance between any two signal sequences. Since the two quadrature trellis decoders are identical and independent in AWGN, the analysis is restricted without loss of generality to the inphase trellis decoder. Since the channel for 
the inphase decoder is a linear intersymbol channel, the difference signal between any two possible signal sequences is equivalently the signal modulated by the difference sequence (or error sequence) of those two inphase information sequences [27]. In general, depending on the severity and type of intersymbol interference, the determination of the error sequence that gives rise to the minimum distance is very difficult. Here, however, it is reasonable to propose, that since the channel filter has no spectral nulls and the trellis has memory length of 2 , the minimum error sequence is $2,0,0, \ldots$. This proposition is verified later by computer simulations.

The probability of error event $\left(\mathrm{P}_{\mathrm{e}}\right)$ for the inphase trellis decoder at moderate to high signal to noise ratio is:

$$
P_{e} \cong Q\left(\sqrt{d_{\text {min }}^{2} E_{s} / 4 \sigma_{n}^{2}}\right)
$$

where $d_{\min }^{2}$ is the normalized minimum distance squared, $E_{s}$ is the energy of the minimum difference signal, and $\sigma_{n}^{2}$ is the variance of the AWGN which is No/2 watts. In the sampled domain, $\mathrm{d}_{\min }^{2}$ caused by the error sequence $2,0, .$. is calculated by:

$$
\mathrm{d}_{\min }^{2}=\frac{1}{2 \mathrm{~W}} \sum_{\mathrm{j}=1}^{\mathrm{L}}\left\|2 \mathrm{~g}_{\mathrm{i}}\right\|^{2}=\frac{2}{\mathrm{~W}} \sum_{\mathrm{j}=1}^{\mathrm{L}}\left\|\mathrm{g}_{\mathrm{i}}\right\|^{2}
$$

where $g_{i, j}=g\left(t=i T_{s y}+j T_{s}\right)=\int_{0}^{T_{s y}} p\left(\tau-T_{b}\right) h(t-\tau) d \tau$. Recall that: $\int_{-\infty}^{\infty} p(t)^{2} d t=1$.

The factor $1 / 2 \mathrm{~W}$ normalizes $d_{\min }^{2}$ to unit energy and thus the baseband noise variance to No. Since the residual ISI is less than $-30 \mathrm{~dB}$, then

$$
\frac{1}{2 W} \sum_{i=0}^{L}\left\|g_{i}\right\|^{2} \cong \int_{-W}^{W}|P(f)|^{2} d f
$$

where $P(f)=\int_{-\infty}^{\infty} p(t) \exp (-i 2 \pi f t) d t$. Hence $d_{\min }^{2}$ equals: 


$$
\mathrm{d}_{\min }^{2}=4 \int_{-\mathrm{W}}^{\mathrm{W}}|\mathrm{P}(\mathrm{f})|^{2} \mathrm{df}
$$

Recall that without ISI, where the optimum performance in AWGN is achieved with the MF, $d_{\min }^{2}$ equals 4. Thus the performance degradation of $d_{\min }^{2}$ in $d B$ for the inphase trellis decoder compared to the MF performance in AWGN is :

$$
\operatorname{10log}\left(\int_{-w}^{w}|P(f)|^{2} d f\right) \quad d B
$$

Equation (4.5) indicates that the more bandwidth efficient the MSK pulse is in terms of minimizing the out of band power radiation at a particular bandwidth $\mathrm{W}$, the smaller the corresponding performance degradation is for the BTR. Table I shows $d_{\text {min }}^{2}$ $\mathrm{dB}$ losses calculated for OQPSK, MSK, SFSK with $\mathrm{W}=0.5 / \mathrm{T}_{\mathrm{b}}$, and $\mathrm{W}=0.25 / \mathrm{Tb}$ respectively.

\begin{tabular}{|c|c|c|}
\hline & $\mathrm{W}=0.5 / \mathrm{T}_{\mathrm{b}}$ & $\mathrm{W}=0.25 / \mathrm{T}_{\mathrm{h}}$ \\
\hline OQPSK & $0.457 \mathrm{~dB}$ & $1.114 \mathrm{~dB}$ \\
\hline SFSK & $0.342 \mathrm{~dB}$ & $2.147 \mathrm{~dB}$ \\
\hline MSK & $0.132 \mathrm{~dB}$ & $1.561 \mathrm{~dB}$ \\
\hline
\end{tabular}

Table I BTR $\mathrm{d}_{\min }^{2}$ loss in $\mathrm{dB}$ for $\mathrm{W}=0.5 / \mathrm{T}_{\mathrm{b}}$, and $\mathrm{W}=0.25 / \mathrm{T}_{\mathrm{b}}$ compared to $\mathrm{MF}$.

The degradation factor shows that the BTR performance is sensitive to bandwidth. For narrow bandwidths, such as $0.25 / \mathrm{T}_{\mathrm{b}}$, even though the AWGN is narrowly filtered, there is a net performance degradation dependent on the bandwidth. This feature suggests that a BTR with a narrow bandwidth $\left(0.25 / \mathrm{T}_{\mathrm{b}}\right)$ compared to a wider bandwidth $\left(0.5 / \mathrm{T}_{\mathrm{b}}\right)$ may not be as good at rejecting ACI because of the poor trade off between the reduction of interference power and the reduction in $\mathrm{d}_{\min }^{2}$. This trend is demonstrated when the performance of the BTR in AWGN and ACI is considered.

As an interesting aside, Wittke and Deshpande [1] have determined the optimum MSK type pulses according to the criterion of minimizing the out of band radiation for a 
particular bandwidth $\mathrm{W}$. Of interest is that their research reveals that for the bandwidth $\mathrm{W}=0.5 / \mathrm{T}_{\mathrm{b}}$ the MSK pulse is very close to the optimum, whereas for $0.25 / \mathrm{T}_{\mathrm{b}}$ OQPSK is close to optimum.

Note that since the AWGN is uncorrelated and hence statistically independent in each quadrature branch, then the probability of error event $\left(\mathrm{P}_{\mathrm{e}}\right)$ in the inphase trellis decoder is independent of the probability of error event in the quadrature trellis decoder (which has the same probability). Each error event has 1 bit in error. Thus the overall bit error rate $P_{b}$ equals:

$$
\mathrm{P}_{\mathrm{b}}=2 \mathrm{P}_{\mathrm{e}}-\left(\mathrm{P}_{\mathrm{e}}\right)^{2} \cong 2 \mathrm{P}_{\mathrm{e}}
$$

\subsubsection{Simulation Results}

Simulations were conducted according to an exact baseband model of the inphase trellis decoder. Hence all the ISI terms (even those discarded by the trellis decoder) are simulated, and the noise samples are uncorrelated Gaussian random variables with variance 2 NoW. For each $E_{b} /$ No parameter, the simulation length was 2 million, where the binary bit stream was simulated according to a pseudo-random sequence of length $2^{31}-1$. 


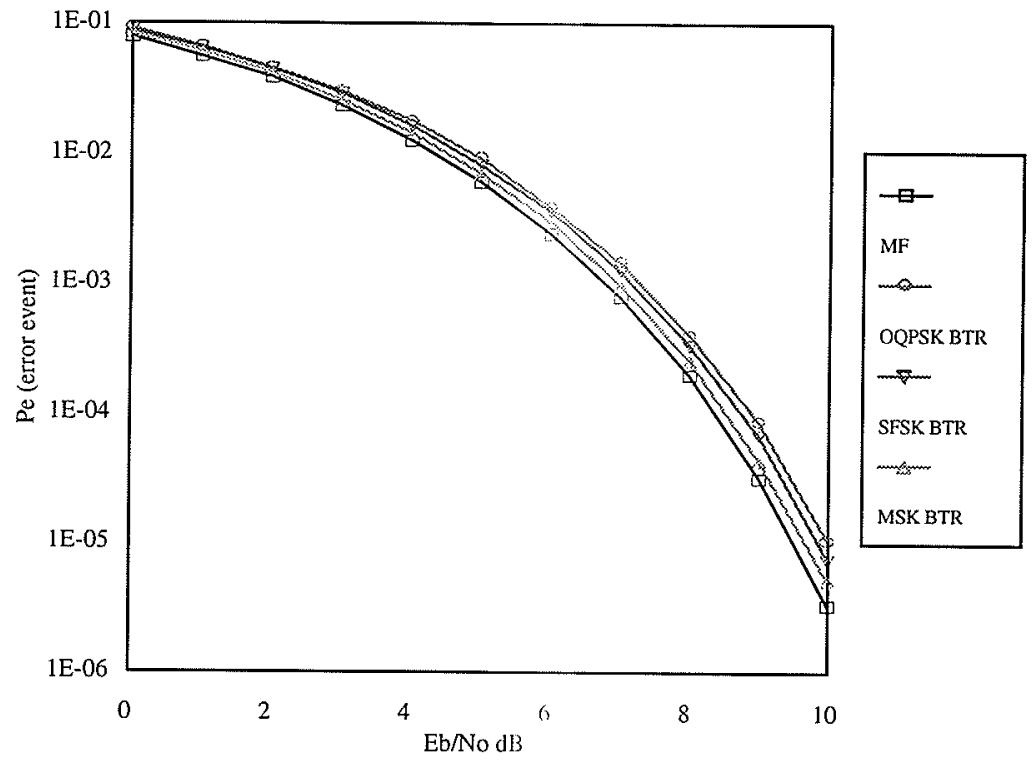

Figure 4-1 BTR performance in AWGN for OQPSK, SFSK and MSK with $\mathrm{W}=0.5 / \mathrm{Tb}$.

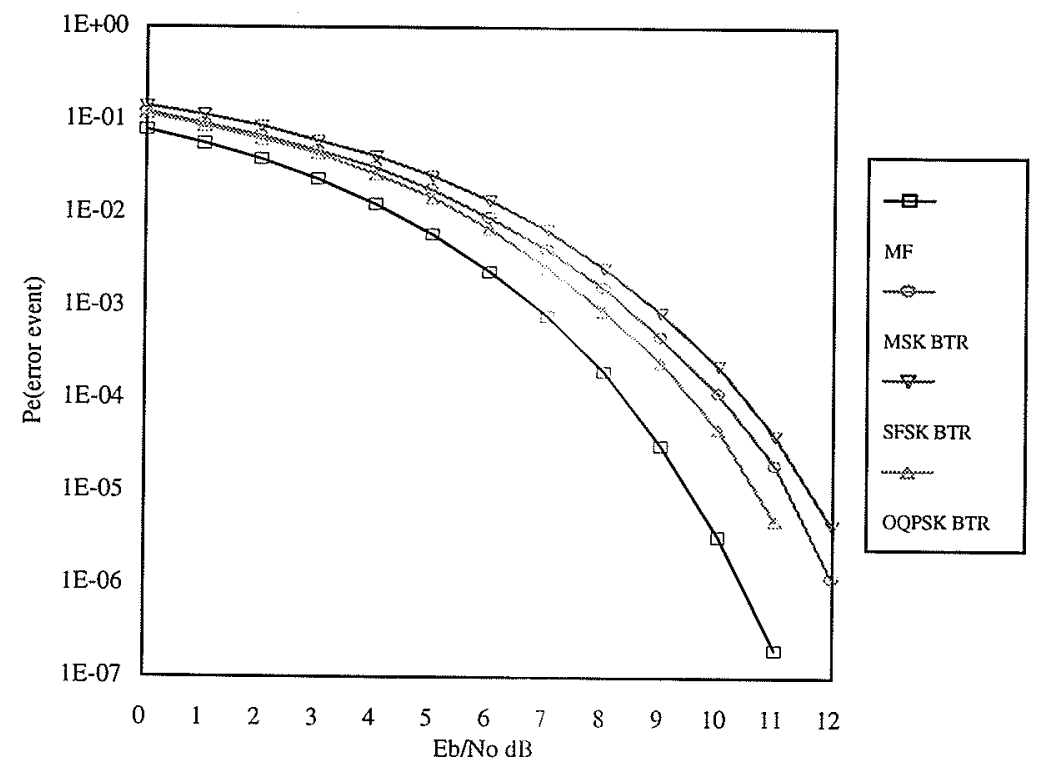

Figure 4-2 BTR performance in AWGN for OQPSK, SFSK and MSK with $\mathrm{W}=0.25 / \mathrm{T}_{\mathrm{b}}$. 
The simulation results shown in Figures $4-1,4-2$ with $\mathrm{W}=0.5 / \mathrm{T}_{\mathrm{b}}$ and $\mathrm{W}=0.25 / \mathrm{T}_{\mathrm{b}}$ for OQPSK, MSK, SFSK agree with the $\mathrm{d}_{\min }^{2} \mathrm{~dB}$ loss indicated by the analysis. The performance of these three modulations for the BTR with $W=0.5 / T_{b}$, but particularly the more bandwidth efficient MSK at $\mathrm{W}=0.5 / \mathrm{T}_{\mathrm{b}}$, are practically indistinguishable from the optimum performance of the matched filter.

\subsubsection{SR revceiver.}

In the case of the filter with $\mathrm{W}=0.5 / \mathrm{T}_{\mathrm{b}}$, sampling the filtered signal at the Nyquist rate introduces at least two serious intersymbol terms. In effect, the trellis decoder is compensating for these ISI terms. A simpler receiver (SR) is one that samples at the symbol rate $\left(2 \mathrm{~T}_{\mathrm{b}}\right)$, avoids the two dominant ISI terms, and makes decisions every symbol interval. Ignoring the degradation due to the residual ISI for the symbol rate sampler, the performance of the SR can be lower bounded by the analysis already given. The upper bound on $\mathrm{d}_{\min }^{2}$ equals:

$$
\mathrm{d}_{\min }^{2} \leq \frac{1}{2 \mathrm{~W}}\left\|2 \mathrm{~g}_{\mathrm{s}}\right\|^{2}=4\left[\frac{1}{2 \mathrm{~W}}\left\|\mathrm{~g}_{\mathrm{s}}\right\|^{2}\right]
$$

where $g_{s}$ is the peak sample at the output of channel $h(t)$ due to $p(t)$.

The degradation of $\mathrm{d}_{\min }^{2}$ compared to the matched filter is given in Table II.

Table II. SR $\mathrm{d}_{\min }^{2}$ loss in $\mathrm{dB}$ for $\mathrm{W}=0.5 / \mathrm{T}_{\mathrm{b}}$, and $\mathrm{W}=0.25 / \mathrm{T}_{\mathrm{b}}$

\begin{tabular}{|c|c|c|c|c|}
\hline & \multicolumn{2}{|c|}{$\mathrm{SR} \mathrm{d}_{\min }^{2}$ loss to $\mathrm{MF}$} & \multicolumn{2}{|c|}{$\mathrm{SR}_{\min }^{2}$ loss to BTR } \\
\hline & $W=0.5 / T_{h}$ & $\mathrm{~W}=0.25 / \mathrm{T}_{\mathrm{b}}$ & $W=0.5 / T_{h}$ & $\mathrm{~W}=0.25 / T_{h}$ \\
\hline OQPSK & $0.161 \mathrm{~dB}$ & $1.121 \mathrm{~dB}$ & $1.153 \mathrm{~dB}$ & $0.007 \mathrm{~dB}$ \\
\hline MSK & $0.534 \mathrm{~dB}$ & $1.57 \mathrm{~dB}$ & $0.402 \mathrm{~dB}$ & $0.01 \mathrm{~dB}$ \\
\hline
\end{tabular}


Table II indicates that the BTR has superior performance to the SR for $\mathrm{W}=0.5 / \mathrm{T}_{\mathrm{b}}$, but for $\mathrm{W}=0.25 / \mathrm{T}_{\mathrm{b}}$ has indistinguishable performance. The filtered signal of interest is effectively frequency equalized with $\mathrm{W}=0.25 / \mathrm{T}_{\mathrm{b}}$. The cost, however, is significant $\mathrm{d}_{\min }^{2}$ degradation compared to $\mathrm{W}=0.5 / \mathrm{T}_{\mathrm{b}}$, which results in poor performance in AWGN and in ACI (shown later).

\subsection{BTR PERFORMANCE IN AWGN AND ACI}

\subsubsection{Error Analysis}

There has been error analysis presented for the MF detection of MSK in ACI and AWGN [23]-[24], as well as for the Viterbi detection of continuous phase modulations in ACI and AWGN[25]. Only an outline of these analyses are presented here in order to emphasize the different sensitivity between the MF and the BTR on the statistics of the ACI. The performance of the BTR and MF in ACI and AWGN are then estimated from Monte Carlo simulations, with some results compared with the previous studies to confirm reliability of the simulations.

For discussion, the equivalent baseband received signal $r(t)$ for the inphase branch in AWGN and $\mathrm{ACI}$ is:

$$
\begin{gathered}
r(t)=A s(t)+a(t)+n(t) \\
s(t)=A \sum_{j} a_{i j}^{l} p\left(t-j 2 T_{b}\right) \\
A=\sqrt{2 E_{b} / T_{b}} \\
a(t)=\sum_{i=1}^{N a} \sum_{j} A_{i} a_{i, j}^{I} p_{i}\left(t-j 2 T_{b}-\lambda_{i}\right) \cos \left(2 \pi f d_{i} t+\theta_{i}\right) \\
-\sum_{i=1}^{N a} \sum_{j} A_{i} a_{i, j}^{Q} p_{i}\left(t-j 2 T_{b}-T_{b}-\lambda_{i}\right) \sin \left(2 \pi f d_{i} t+\theta_{i}\right)
\end{gathered}
$$


where $\mathrm{Na}$ is the number of interferers, $\left\{\mathrm{A}_{\mathrm{i}}, \mathrm{fd}_{\mathrm{i}}, \lambda_{\mathrm{i}}, \theta_{\mathrm{i}}\right\}$ are the parameters of each interferer, $\lambda_{i}$ and $\theta_{i}$ are independent uniform random variables over $(0,2 \pi),\left(-T_{b}, T_{b}\right)$ respectively. It is assumed that all the inphase and quadrature bit streams of the interferers $\left\{\mathbf{a}^{. \mathrm{I}}, \mathbf{a}^{\mathrm{Q}}\right\}$ are independent equally likely random variables.

The quadrature correlation filter performs maximum likelihood detection for each quadrature symbol in AWGN. The probability of symbol error, $\mathrm{P}_{\mathrm{e}}$, is the probability that the projection of the received signal, $r(t)$, on the difference signal, $s_{2}(t)-s_{1}(t)$, exceeds a certain threshold. Since the signaling in each branch is antipodal the normalized difference signal is just $p(t)$. In the case of $A W G N$ and $A C l$, then $P_{e}$ equals:

$$
P_{e}=\int_{\alpha} f\{\alpha\} Q\left\{\sqrt{\frac{d_{\text {min }}^{2} E_{b}}{2 N_{0}}+\frac{\alpha}{N_{0}}}\right\} d \alpha
$$

where $f\{\alpha\}$ is the density of $\alpha=\int_{-T_{b}}^{T_{\beta}} p(t) a\left(t, \theta_{i}, \lambda_{i}, \mathbf{a}_{i}^{\mathrm{I}}, \mathbf{a}_{i}^{\mathrm{Q}}\right) d t$ over $\mathrm{i}=1, \ldots, \mathrm{Na}$

The important feature of this result is that $\mathrm{P}_{\mathrm{e}}$ depends on integrating a $\mathrm{Q}$ function which is perturbed by the statistics of the ACI filtered by the MF. Hence $P_{e}$ is sensitive to the statistics of the match-filtered ACI when the variance of the nuisance parameter, $\alpha$,is sufficient to degrade performance.

If one now considers the BTR, which performs MLSE on a bandlimited space in AWGN, then the probability of error event $P_{c}$ for high SNR is now the probability that the projection of the received signal on the minimum bandlimited difference signal exceeds a certain threshold. It has already been verified by computer simulations that the minimum bandlimited difference signal equals:

$$
p(t) \otimes h\left(t+T_{b}\right)
$$

where $\otimes$ denotes convolution. Recall that the metric operations performed in the sampled domain by the VA are equivalent to the those performed in the Euclidean bandlimited space. Thus the $P_{e}$ in the case of AWGN and ACI can be formulated as: 


$$
P e=\int_{\alpha} f\{\hat{\alpha}\} Q\left\{\sqrt{\frac{\hat{d}_{\text {min }}^{2} E_{b}}{2 N_{o}}+\frac{\hat{\alpha}}{N_{o}}}\right\} d \hat{\alpha}
$$

where $f\{\hat{\alpha}\}$ is the density of $\hat{\alpha}=\int_{-15 \mathrm{~T}_{\mathrm{b}}}^{15 \mathrm{~T}_{\mathrm{b}}}\left\{\mathrm{p}(\mathrm{t}) \otimes \mathrm{h}\left(\mathrm{t}+\mathrm{T}_{\mathrm{b}}\right)\right\} \mathrm{a}\left(\mathrm{t}, \theta_{\mathrm{i}}, \lambda_{\mathrm{i}}, \mathbf{a}_{\mathrm{i}}^{\mathrm{I}}, \mathbf{a}_{\mathrm{j}}^{\mathrm{Q}}\right) \mathrm{dt}$ over $\mathrm{i}=1, \ldots, \mathrm{Na}$ and $\hat{d}_{\min }^{2}$ is the degraded $d_{\min }^{2}$ due to the bandlimiting filtering. Since the BTR has more random terms of the adjacent signals in the filtered $\mathrm{ACI}$ due to the timespan of $\mathrm{h}(\mathrm{t})$, the amplitude statistics of the filtered ACI are more Gaussian. The same remarks concerning $P_{e}$ sensitivity to the statistics of the filtered $\mathrm{ACl}$ applies, except that the variance of this filtered ACI should be less because of the additional filtering by the bandlimiting filter $h(t)$.

\subsubsection{An example of the statistics of the filtered ACI}

For illustration purposes, an estimate of $f\{\alpha)$ and $\mathrm{f}\{\hat{\alpha}\}$ are obtained from Monte Carlo simulations for the case of one adjacent MSK signal frequency offset by $2.0 / \mathrm{T}_{\mathrm{b}}$ and at $20 \mathrm{~dB}$ interference power to signal power ratio $(\mathrm{I} / \mathrm{S})$. Comparing the two densities shown in Figures 4-3 and 4-4, $f\{\alpha)$ is seen as wider spread than $f\{\hat{\alpha}\}$, with a spike around zero which indicates that there is a significant range of synchronization and phase offsets for which the ACI is in fact orthogonal to the SOI. If $f\{\alpha)$ and $f \hat{\alpha}\}$ are both normalized to have the same variance, $f\{\alpha)$ will be still wider spread than $f\{\hat{\alpha}\}$, and hence these additional 'tails' of the $f(\alpha)$ will incur more error at the medium to high SNR range. This illustration serves to suggest that $f\{\alpha)$ is relatively more detrimental than $f\{\hat{\alpha}\}$ to error performance in the medium to high SNR range. 


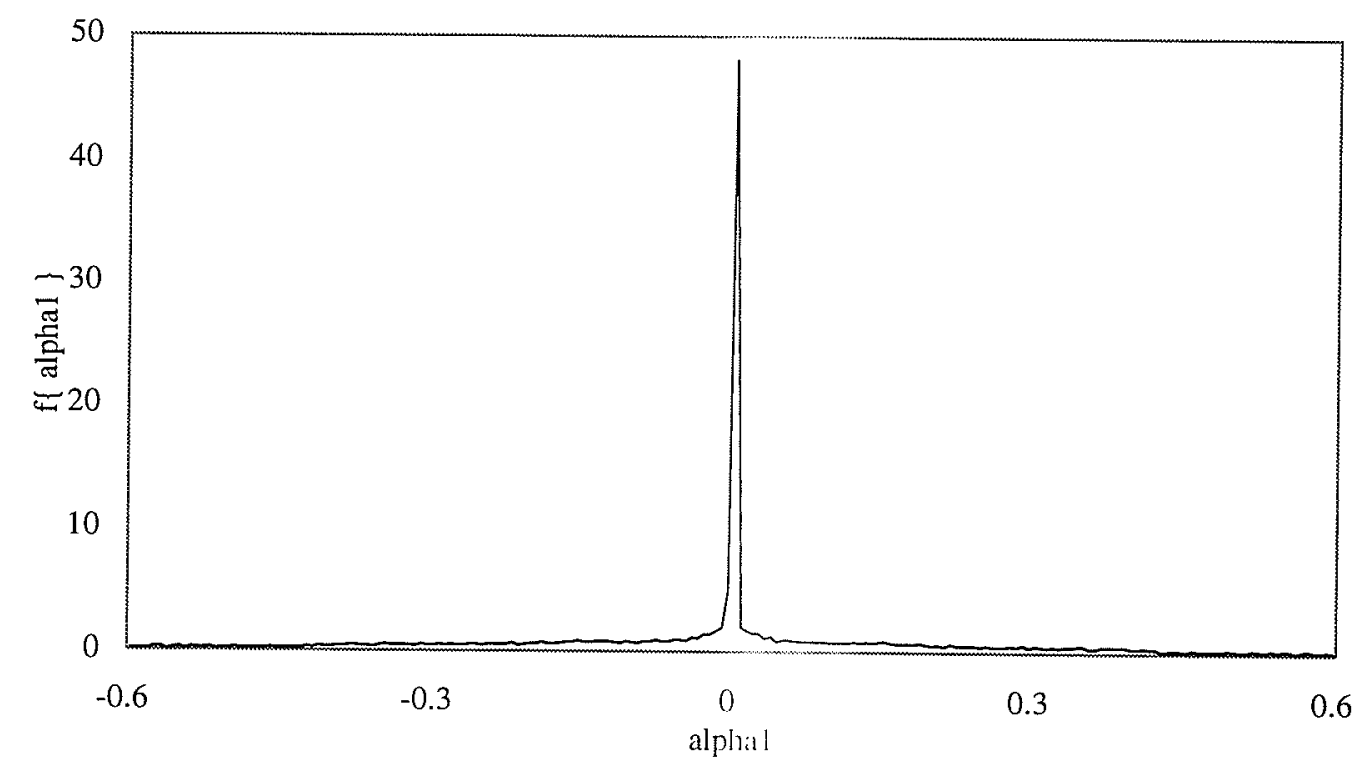

Figure 4-3 Estimate of $\mathrm{f}\{\alpha=$ alpha1) for adjacent MSK $f$ offset at 2.0/T at $20 \mathrm{~dB}$ I/S

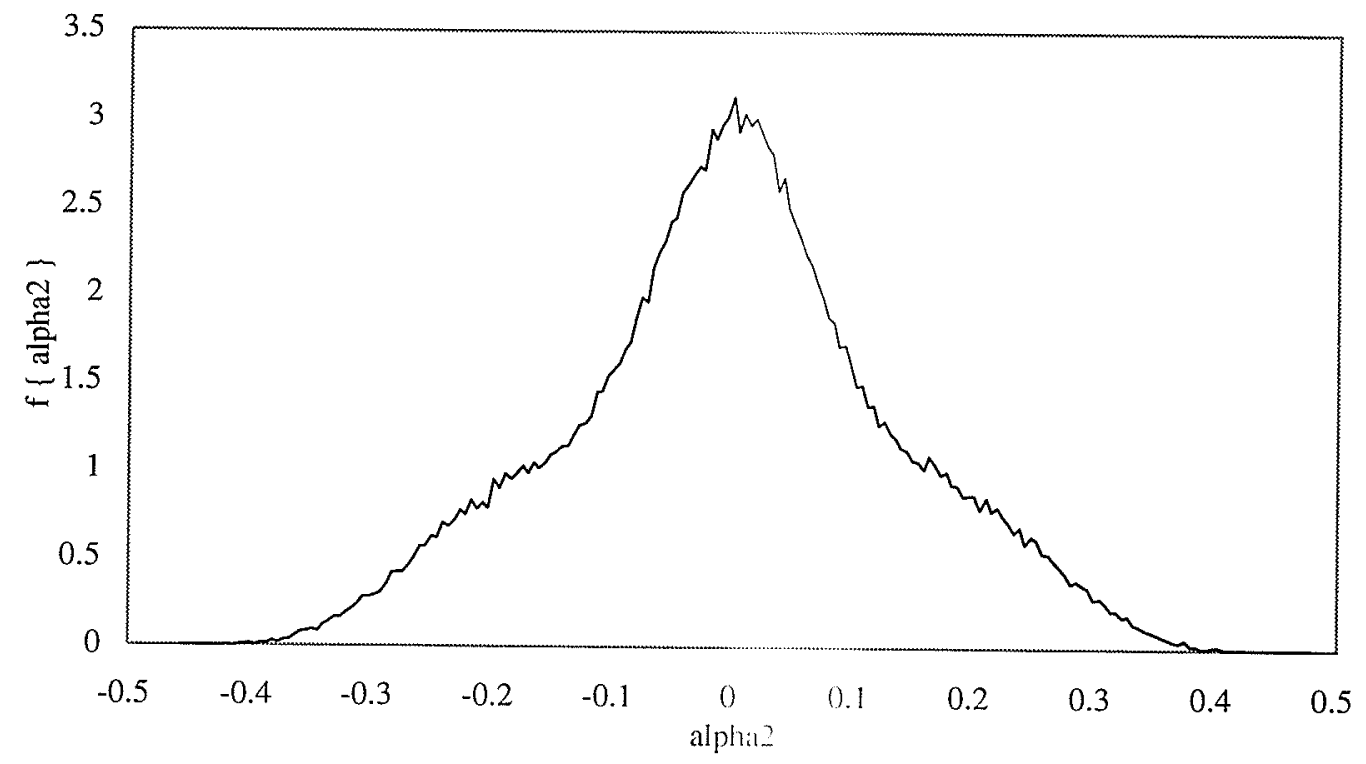

Figure 4-4 Estimate of $f\{\hat{\alpha}=$ alpha2) for adjacent MSK offset at 2.0/T at $20 \mathrm{~dB} \mathrm{I} / \mathrm{S}$ 


\subsubsection{Simplified Analysis: Gaussian model}

A useful analysis to check the performance of the BTR in AWGN and ACI is to model the filtered ACI as AWGN. The probability of error event $P_{e}$ under this model equals:

$$
\left.P e=Q \sqrt{\frac{\hat{d}_{\text {min }}^{2} E_{b}}{2\left(N o+N^{\prime} o\right)}}\right)
$$

where No is the one sided spectral height of the AWGN, $\hat{d}_{\text {min }}^{2}$ is the $d_{\text {min }}^{2}$ degraded by the filtering and $N^{\prime} \mathrm{O}$ is the equivalent spectral height of the filtered interference in the equivalent baseband model. $\mathrm{N}^{\prime} \mathrm{o}$ is computed before the filter by considering one adjacent signal at a frequency offset of $\mathrm{f}_{\mathrm{d}}$, and at an interference to signal ratio of I/S. It is given by:

$$
N^{\prime} O=\frac{(I / S)}{4 T_{b}(2 W)} \int_{-W}^{W}\left|P\left(f-f_{d}\right)\right|^{2}+\left|P\left(-f-f_{d}\right)\right|^{2} d f
$$

\subsubsection{Simulation Parameters}

Monte Carlo simulations are performed on the BTR and MF receivers in an exact baseband model for the SOI and the ACI, where both the ISI terms of the SOI and the interference terms of the $\mathrm{ACI}$ are retained. The simulations are performed under the following parameters:

1)The random parameters $\left\{\lambda, \theta_{i}\right\}$ are drawn according to their respective density functions every 50 symbol intervals.

2)The run length for each $E_{b} /$ No simulation run was 2 million, hence providing a reliable estimate of error performance up to $5 \times 10^{-6}$ ( 10 errors).

3) The length of each pseudo-random antipodal information sequence is $2^{31}-1$. 
The simulations are conducted for MSK and OQPSK for one interferer at $f_{d}$ equal to $2.0 / \mathrm{T}_{\mathrm{b}}, 1,5 / \mathrm{T}_{\mathrm{b}}$, and $1.0 / \mathrm{T}_{\mathrm{b}}$. The BTR is simulated for both $\mathrm{W}=0.5 / \mathrm{T}_{\mathrm{b}}$ and $\mathrm{W}=0.25 / \mathrm{T}_{\mathrm{b}}$. One interferer at a relatively high $\mathrm{I} / \mathrm{S}$ is the most probable near-far situation.

The simulation results are given in terms of average bit error rate for each quadrature branch. Note that errors in each branch in ACI are no longer statistically independent. Thus there may be some benefit in high ACI for the BTR to exploit the correlation between the quadrature branches.

The simulation results are presented as follows. For each frequency spacing $f_{d}$, the results for the BTR compared to the analytical results based on modeling the ACI as Gaussian are presented first. Then for each $f_{d}$ a plot is given. This plot compares the $\mathrm{E}_{\mathrm{b}} / \mathrm{No} \mathrm{dB}$ loss relative to the matched filter at a $\mathrm{P}_{\mathrm{e}}=10^{-5}$ in no $\mathrm{ACI}$ for each receiver at different levels of $I / S$. This plot illustrates the relative levels of $I / S$ that each receiver sustains before serious performance degradation. 


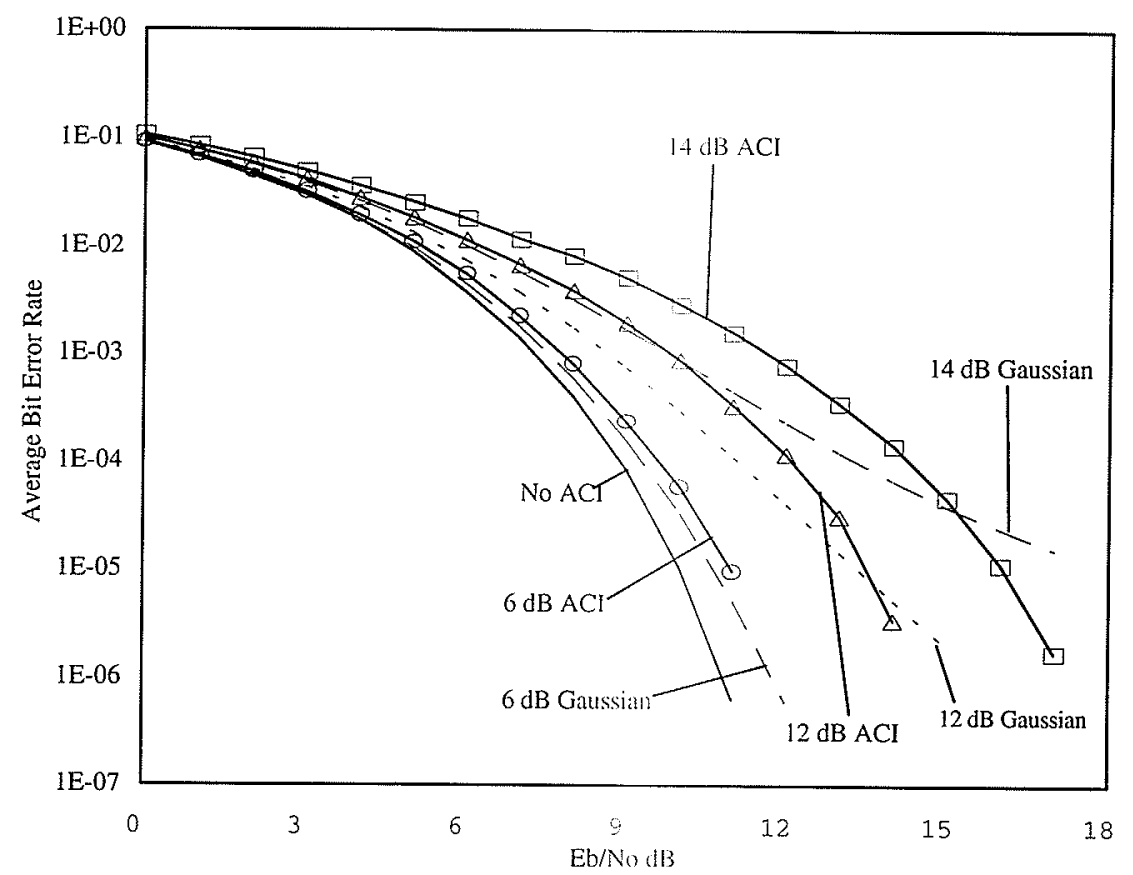

Figure 4-5 OQPSK BTR $W=0.5 / T_{b}$ with one interferer at $f_{d}=2.0 / T_{b}$ and Gaussian model.

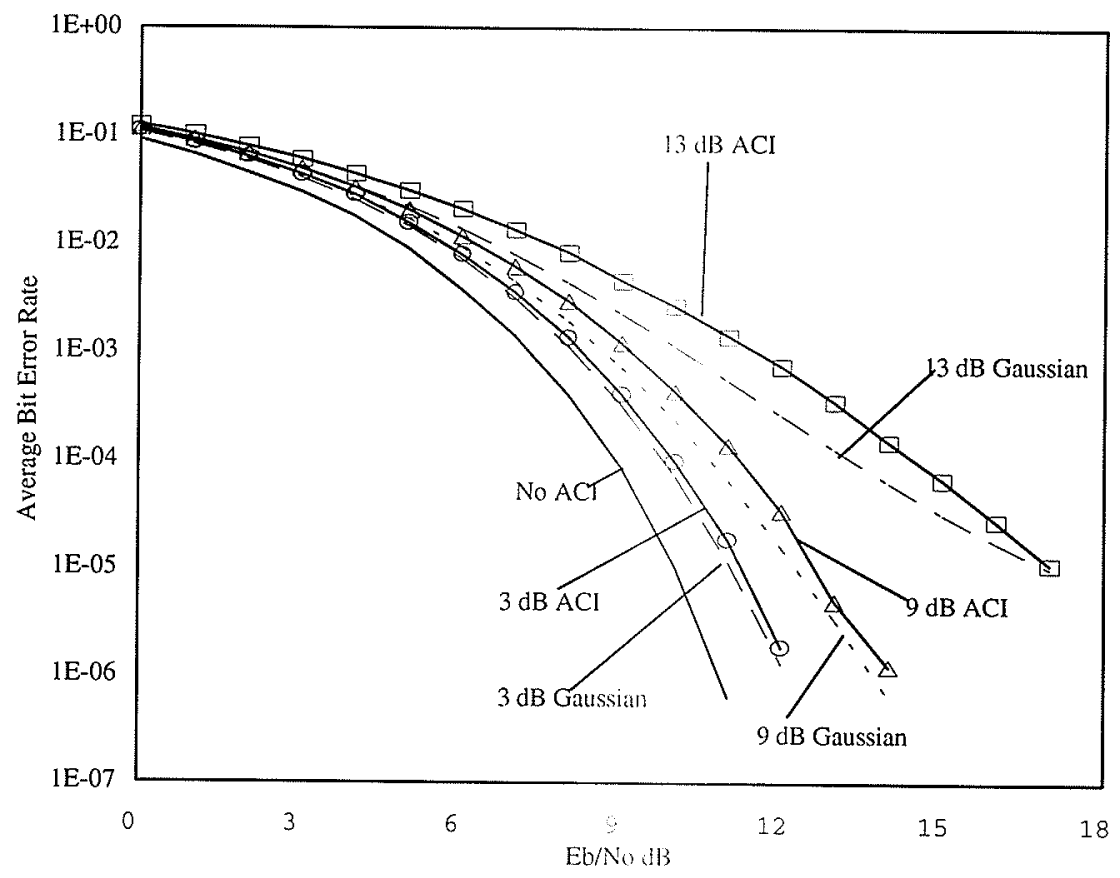

Figure 4-6 OQPSK BTR $W=0.25 / T_{b}$ with one interferer at $f_{d}=2.0 / T_{b}$ and Gaussian Model 


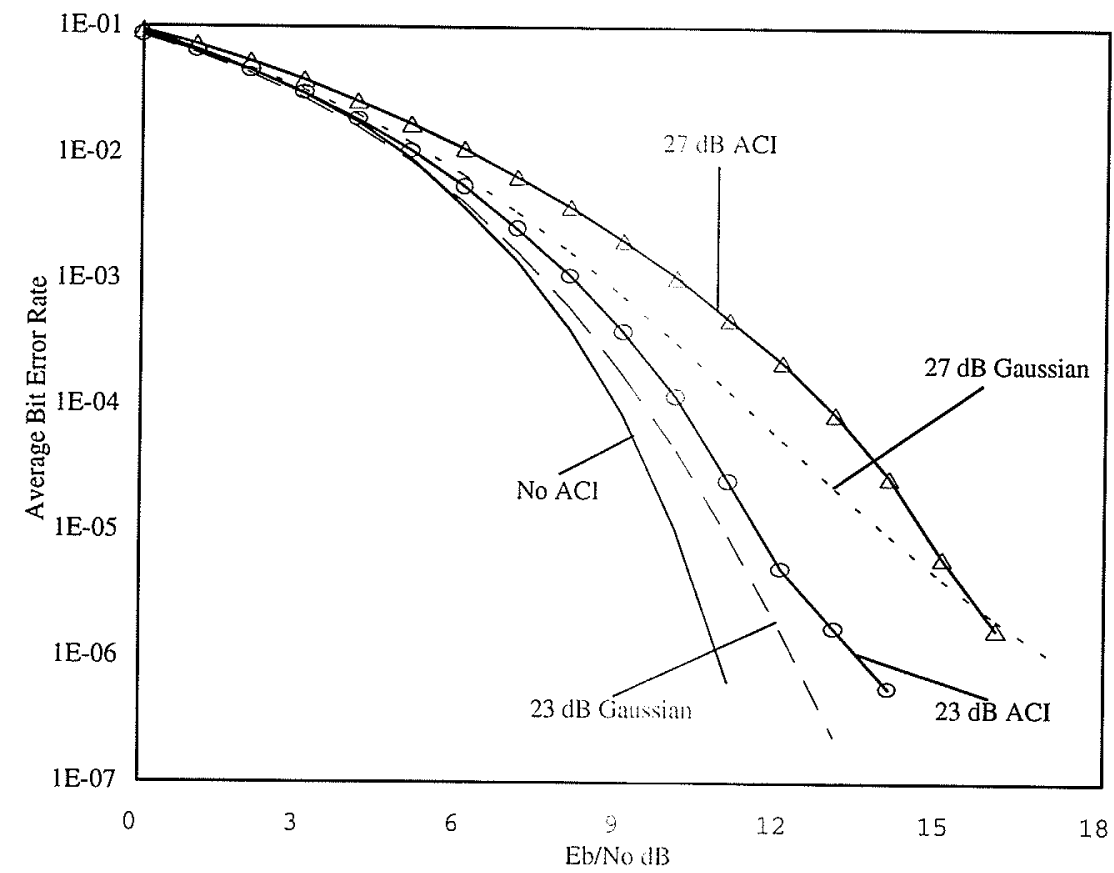

Figure 4-7 MSK BTR $W=0.5 / T_{b}$ with one interferer at $f_{d}=2.0 / T_{b}$ and Gaussian model

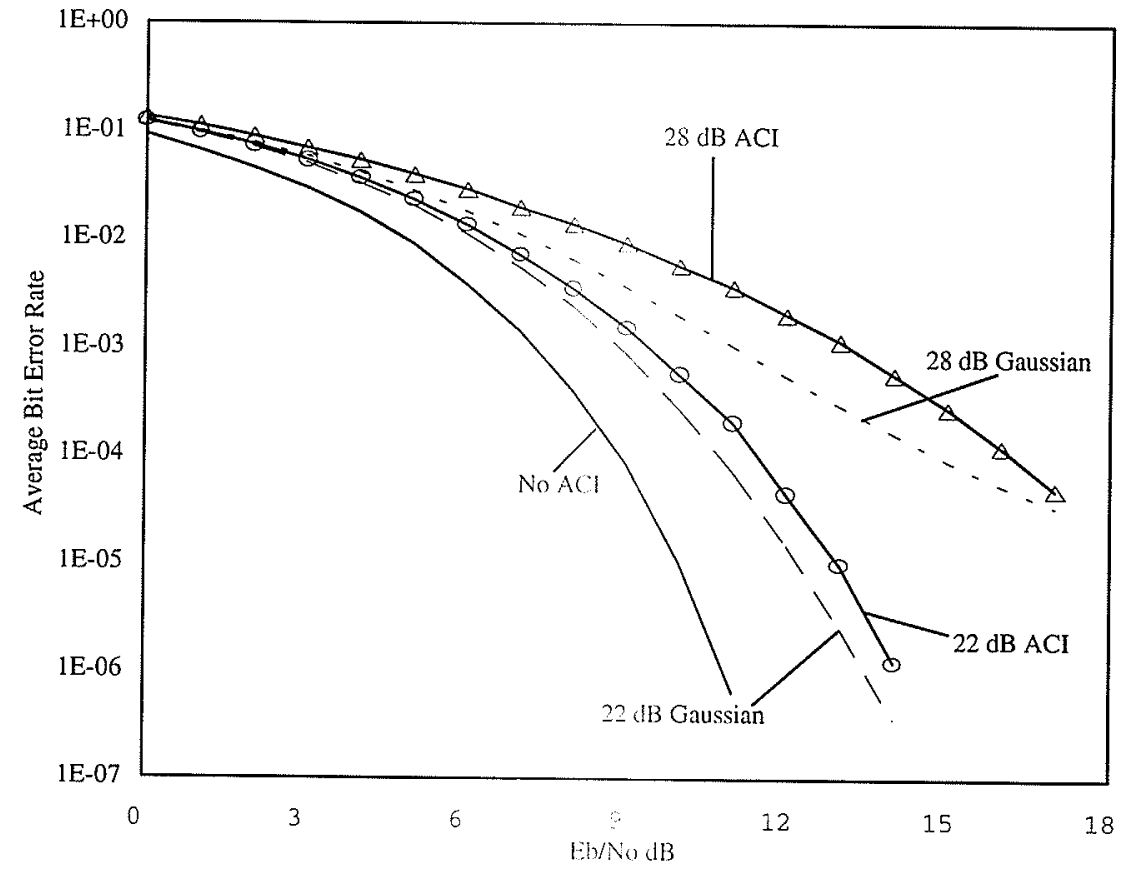

Figure 4-8 MSK BTR $W=0.25 / T_{b}$ with one interferer at $f_{d}=2.0 / T_{b}$ and Gaussian model. 


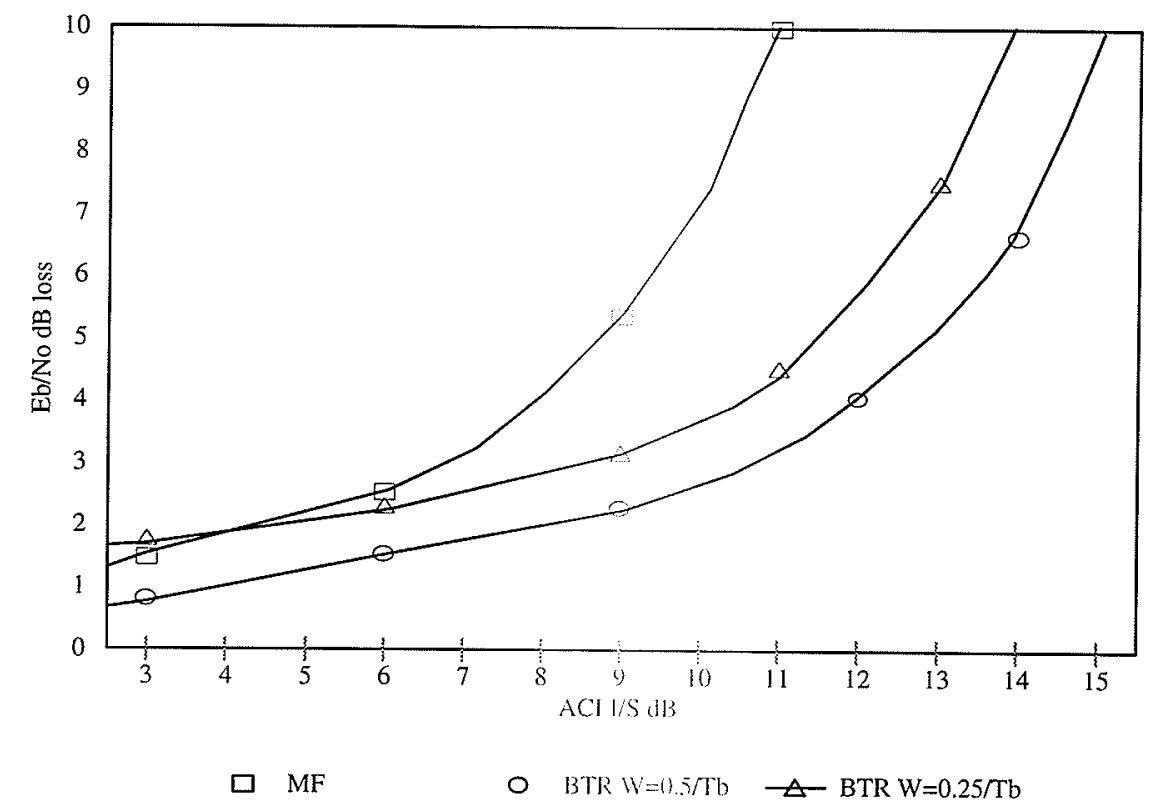

Figure 4-9 $E_{b} /$ No dB loss compared to MF with no $A C I$ at $P_{e}=10^{-5}$ for one interferer at $f_{d}=2.0 / T_{b}$ versus $I / S d B$ for OQPSK

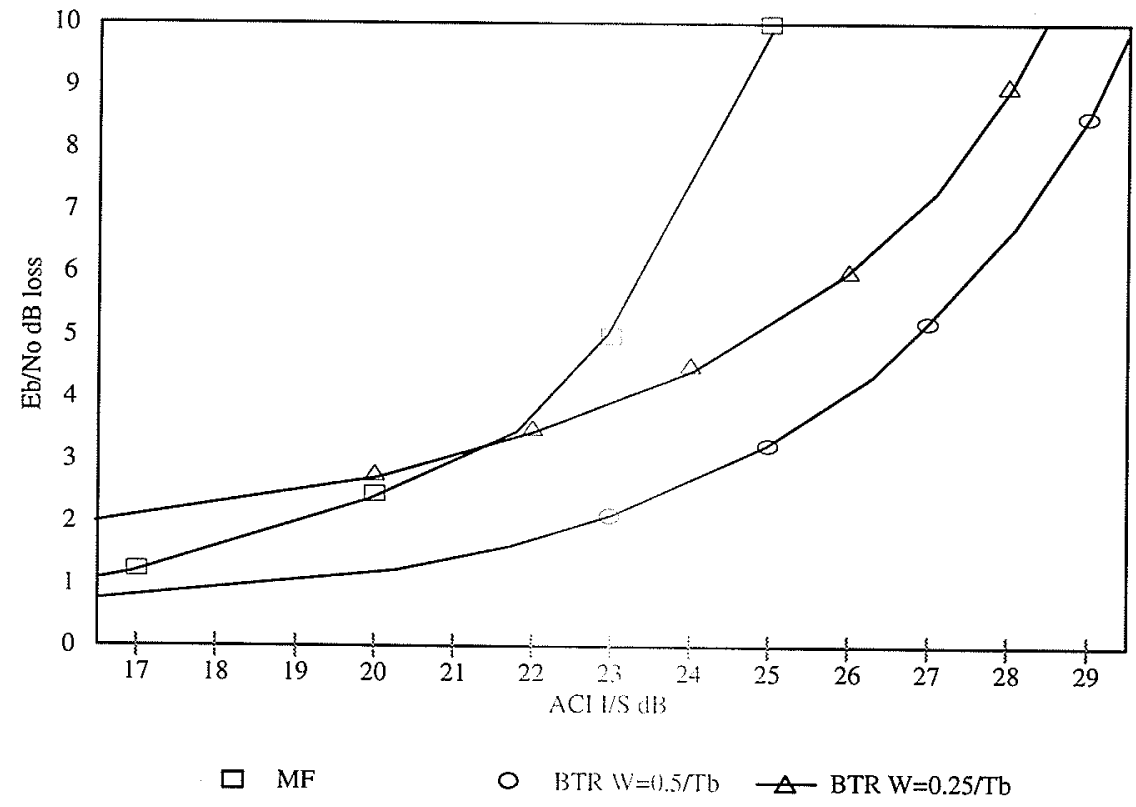

Figure 4-10 $\mathrm{E}_{\mathrm{b}}$ /No $\mathrm{dB}$ loss compared to $\mathrm{MF}$ with no ACI at $\mathbf{P}_{\mathrm{e}}=10^{-5}$ for one interferer at $f_{d}=2.0 / T_{1}$ versus $I / S$ dB for MSK 


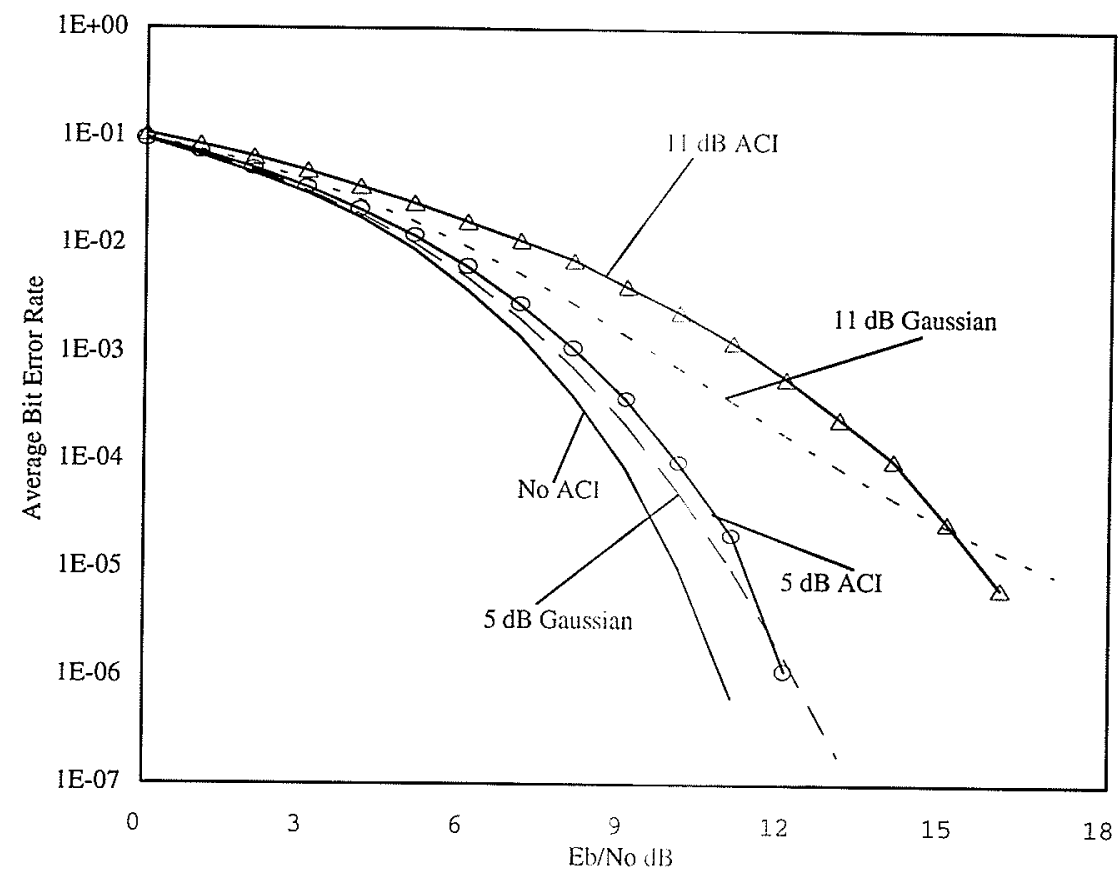

Figure 4-11 OQPSK BTR $W=0.5 / T_{b}$ with one interferer at $f_{d}=1.5 / T_{b}$ and Gaussian model.

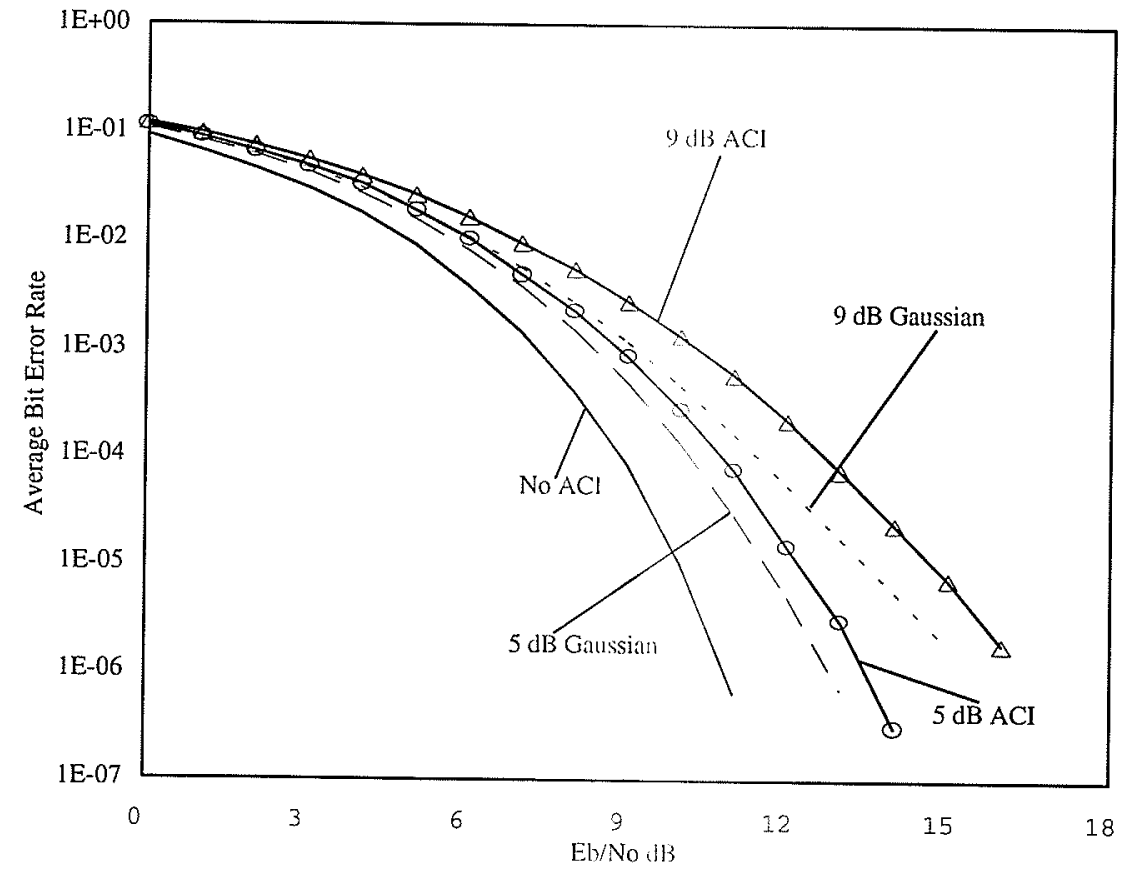

Figure 4-12. OQPSK BTR $W=0.25 / T_{b}$ with one interferer at $f_{d}=1.5 / T_{b}$ and Gaussian model 


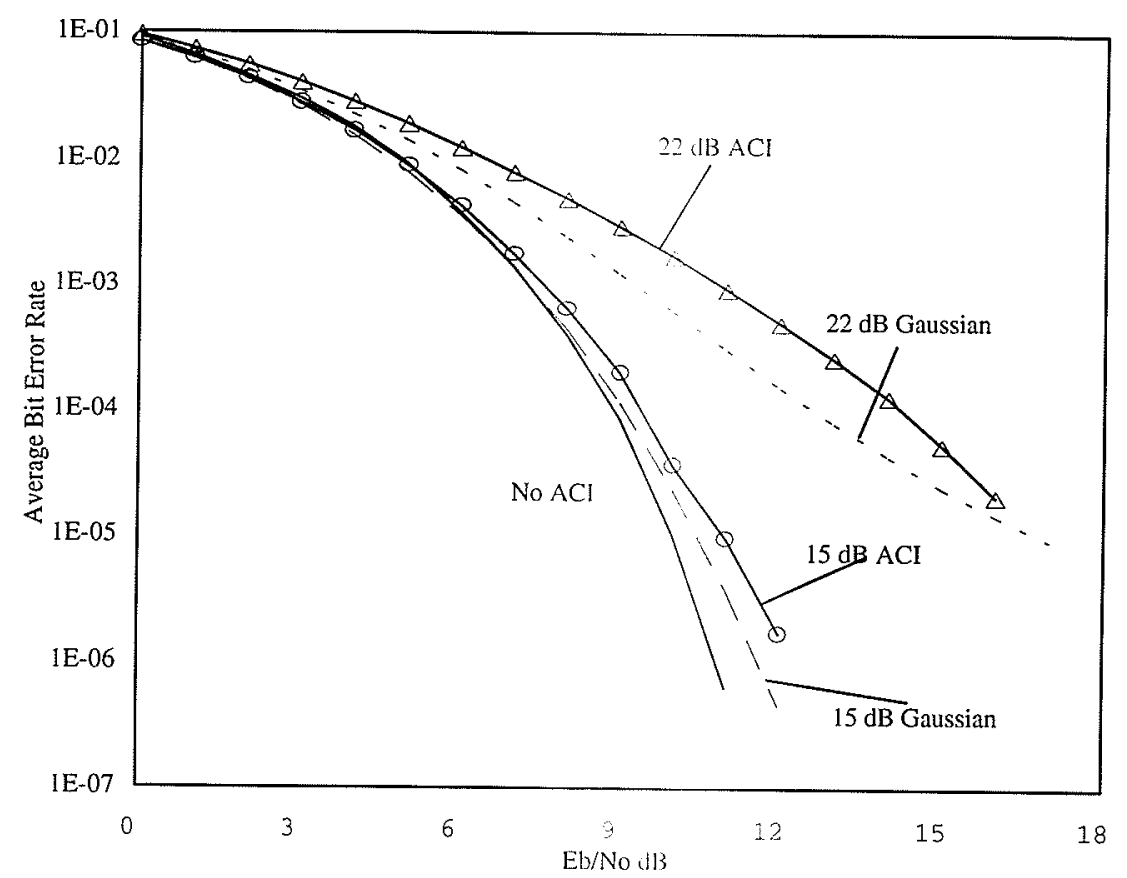

Figure 4-13. MSK BTR $W=0.5 / T_{b}$ with one interferer at $f_{d}=1.5 / T_{b}$ and Gaussian model

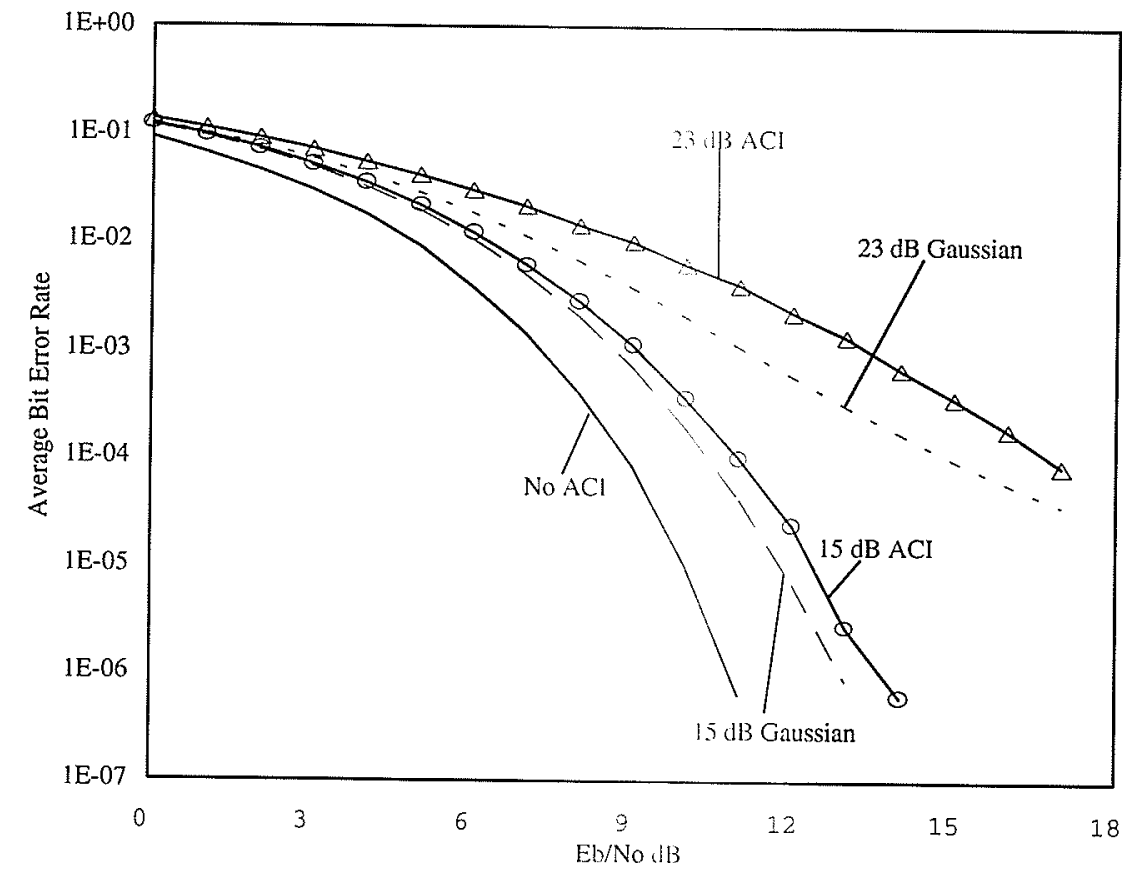

Figure 4-14. MSK BTR $W=0.25 / T_{b}$ with one interferer at $f_{d}=1.5 / T_{b}$ and Gaussian model 


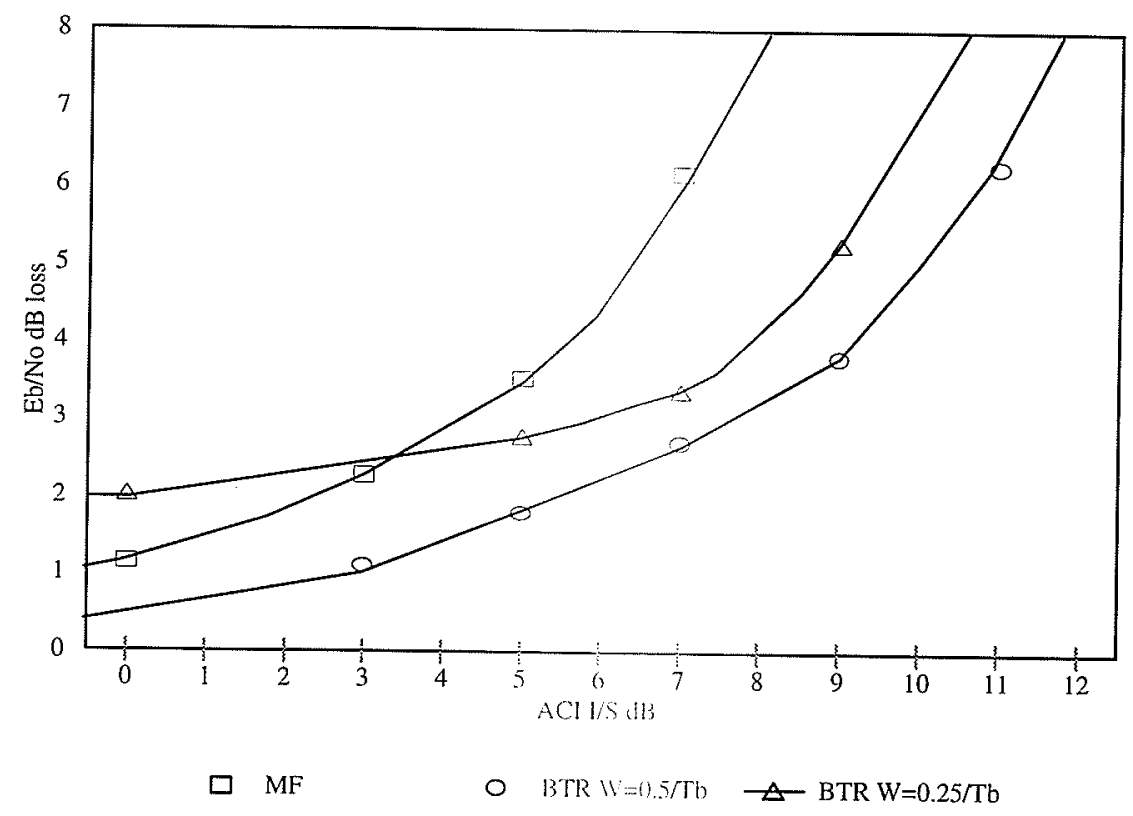

Figure 4-15. $E_{b} /$ No dB loss compared to $M F$ with no $A C I$ at $P_{e}=10^{-5}$ for one interferer at $f_{d}=1.5 / T_{b}$, versus $I / S \mathrm{~dB}$ for OQPSK.

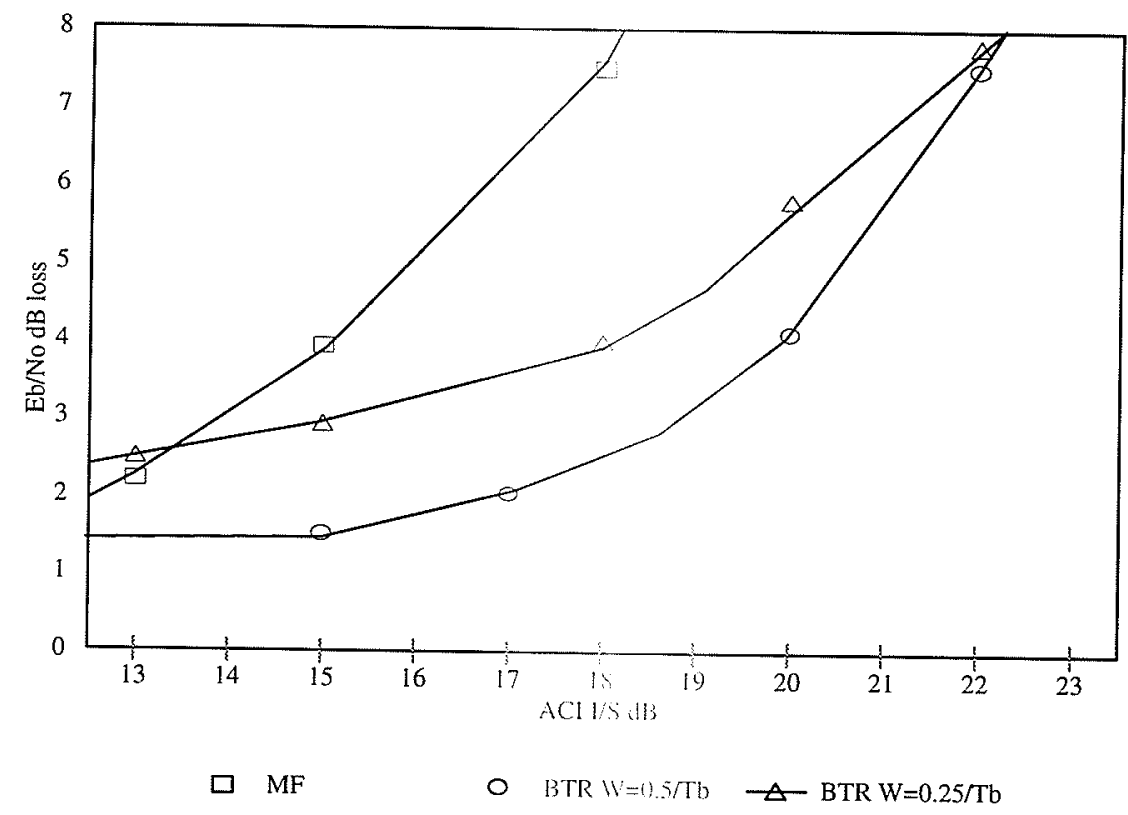

Figure 4-16. $E_{b} /$ No dB loss compared to MF with no ACI at $P_{e}=10^{-5}$ for one interferer at $f_{d}=1.5 / T_{b}$ versus $I / S ~ d B$ for MSK 


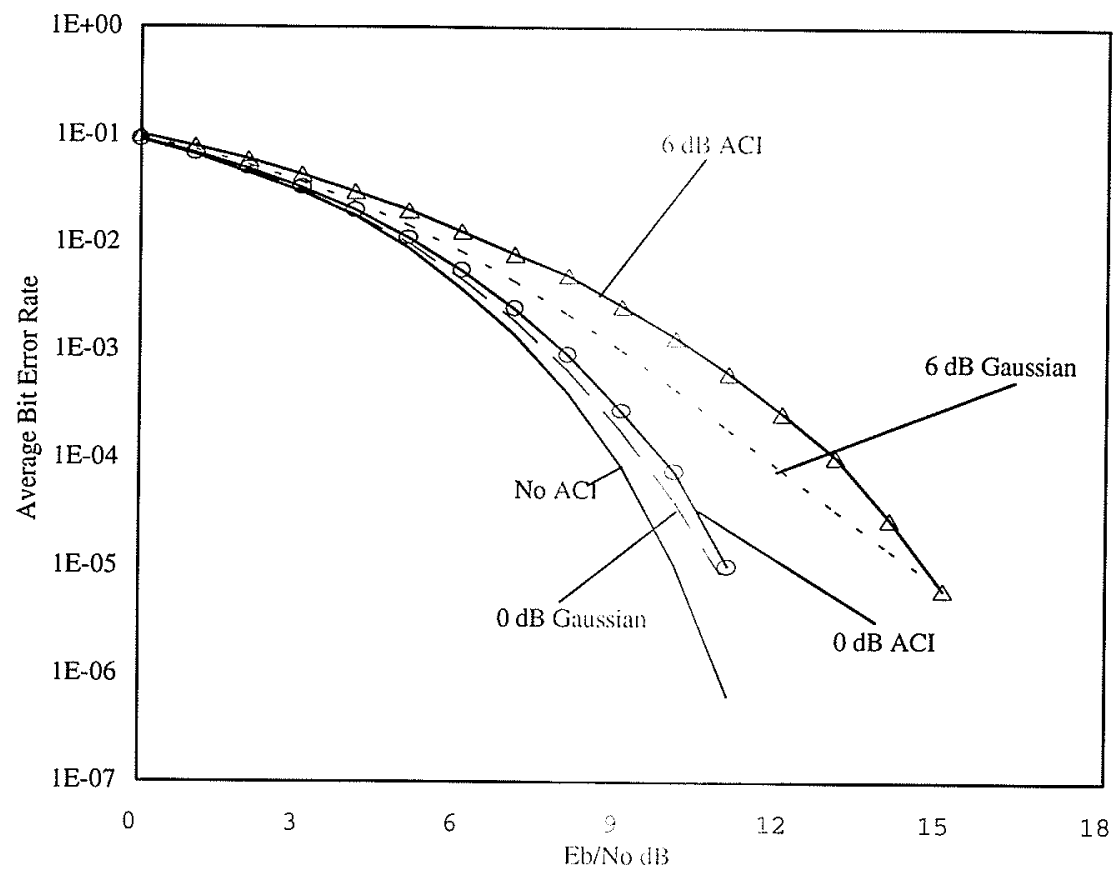

Figure 4-17. OQPSK BTR $W=0.5 / T_{b}$ with one interferer at $f_{d}=1.0 / T_{b}$ and Gaussian Model

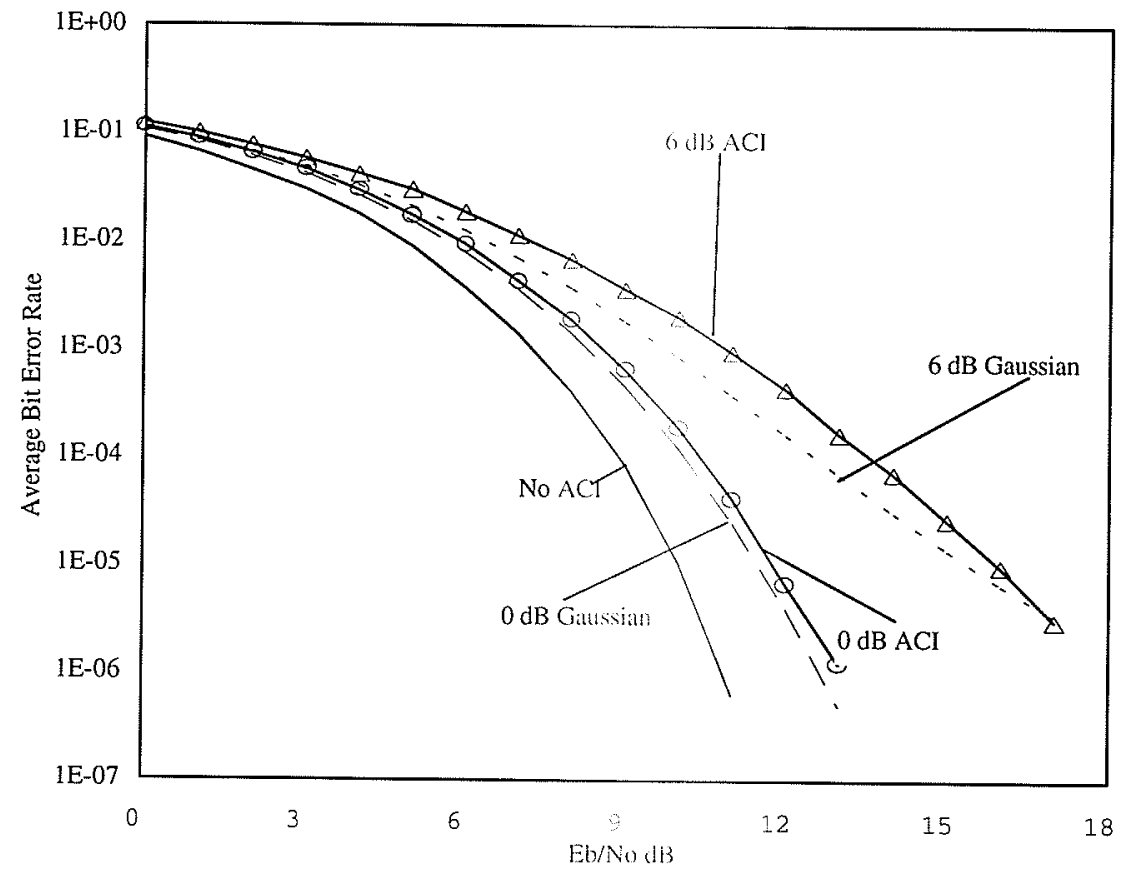

Figure 4-18 OQPSK BTR $W=0.25 / T_{b}$ with one interferer at $f_{d}=1.0 / T_{b}$ and Gaussian model 


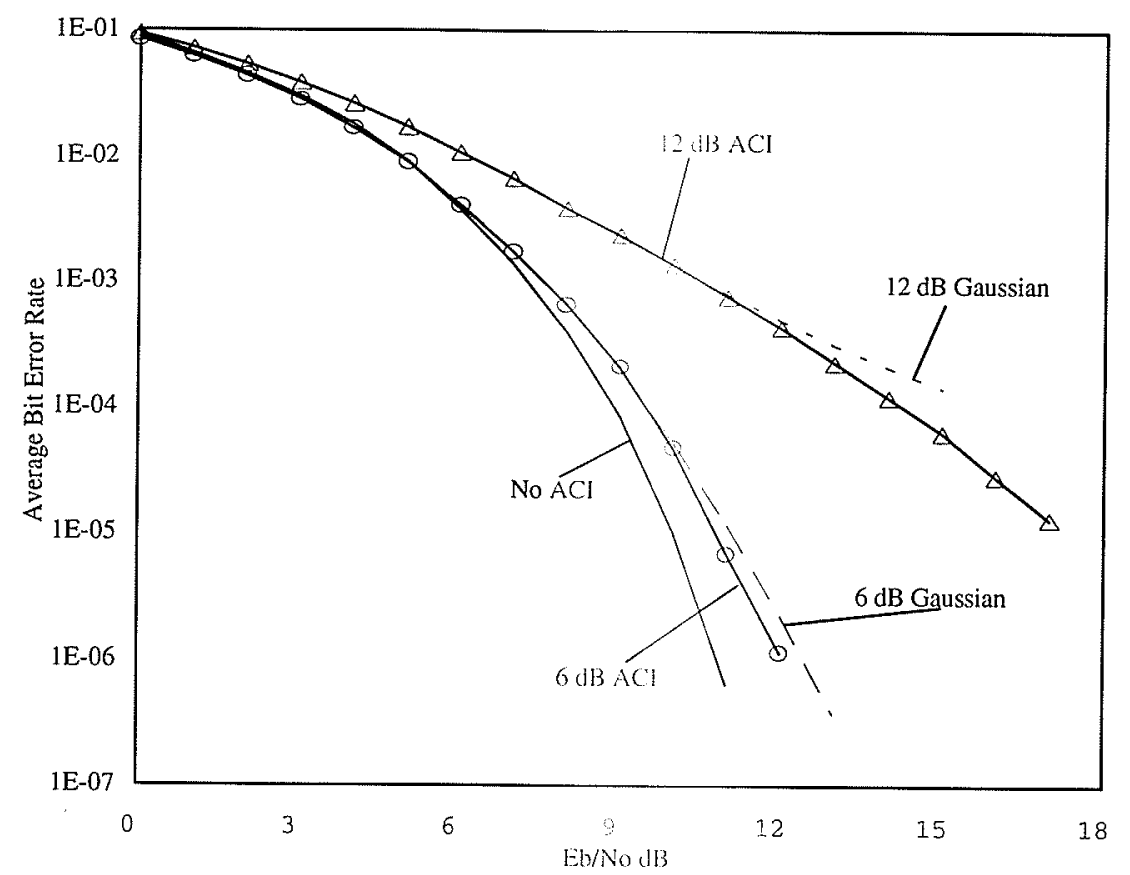

Figure 4-19. MSK BTR $W=0.5 / T_{b}$ with one interferer at $f_{d}=1.0 / T_{b}$ and Gaussian model

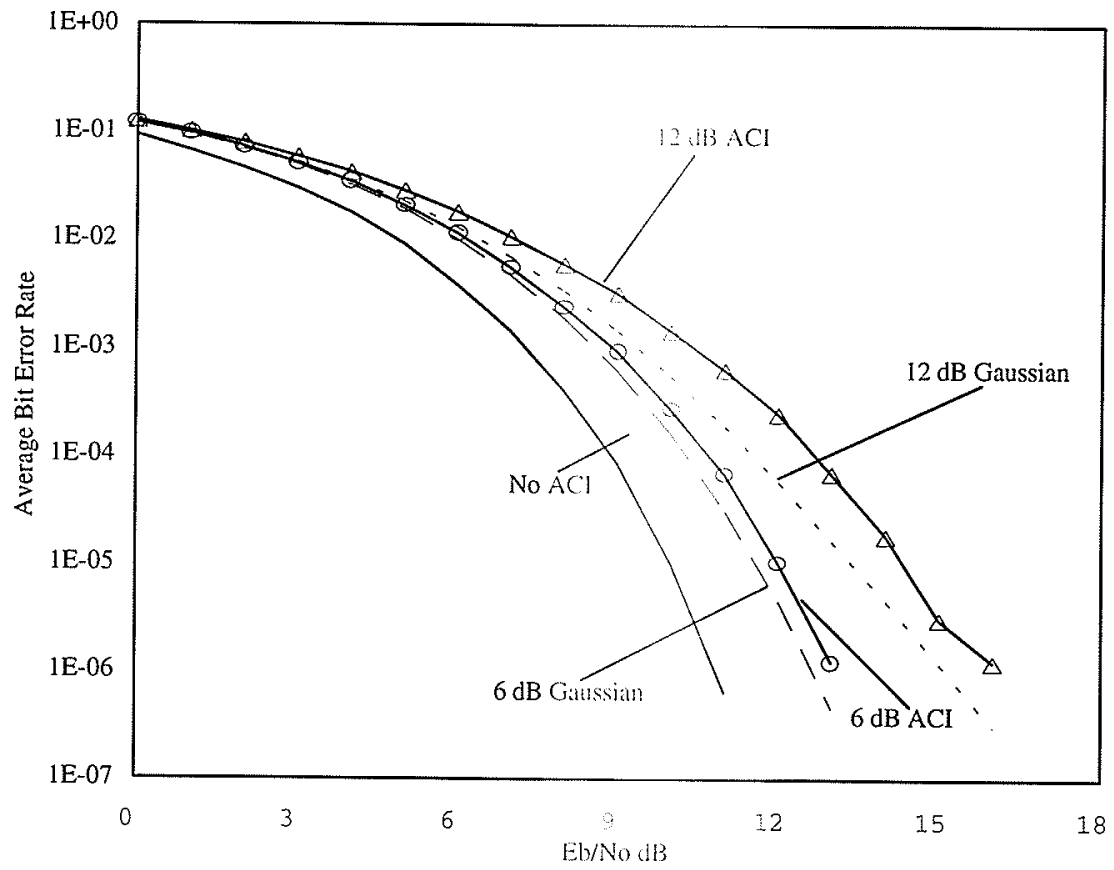

Figure 4-20. MSK BTR $W=0.25 / T_{b}$ with one interferer at $f_{d}=1.0 / T_{b}$ and Gaussian model 


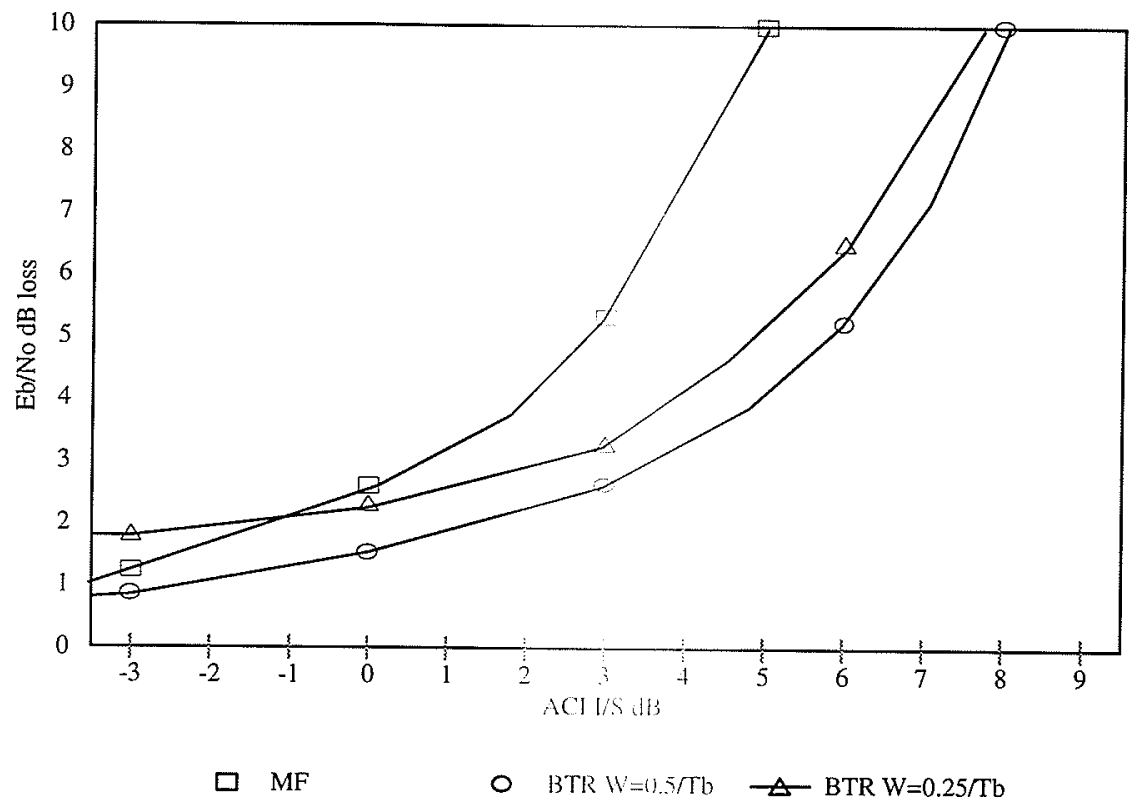

Figure 4-21 $E_{b} /$ No dB loss compared to MF with no $A C I$ at $P_{e}=10^{-5}$ for one interferer at $\mathbf{f}_{d}=\mathbf{1 . 0} / T_{b}$ verstis $\mathrm{I} / \mathrm{S} \mathrm{dB}$ for OQPSK

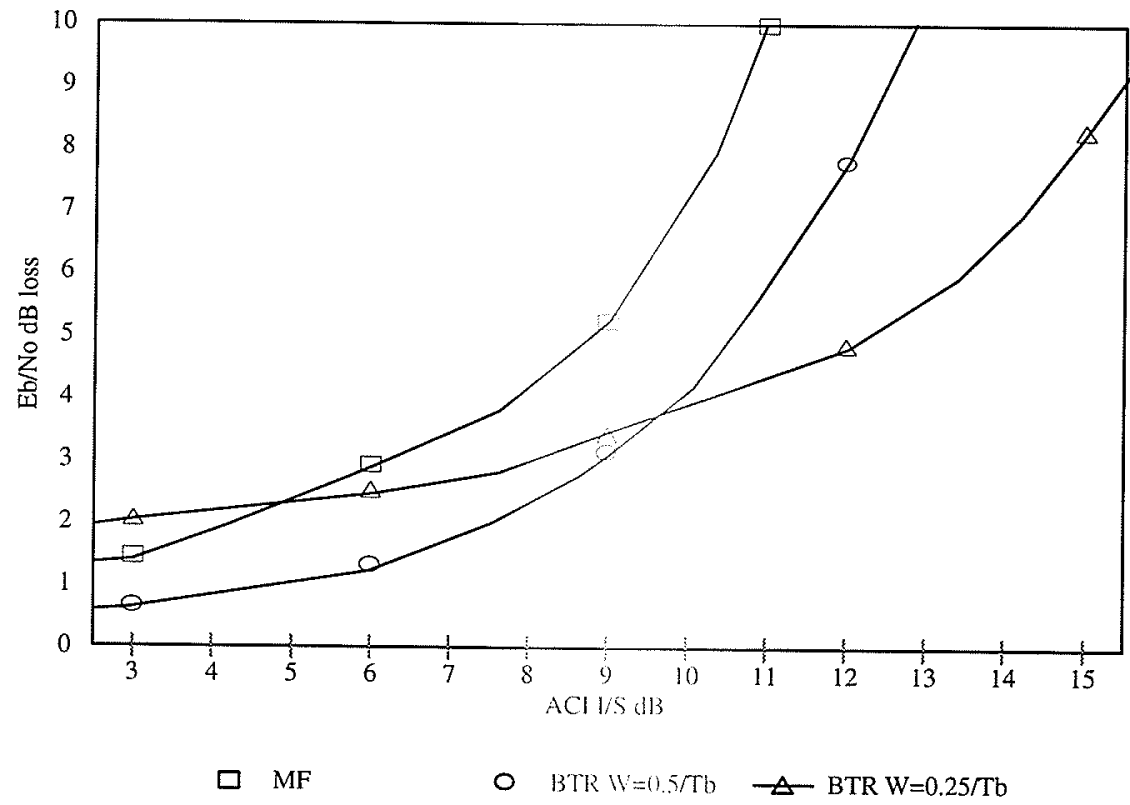

Figure 4-22 $E_{b} /$ No dB loss compared to $\mathrm{MF}$ with no $\mathrm{ACI}$ at $\mathbf{P}_{\mathrm{e}}=10^{-5}$ for one interferer at $\mathbf{f}_{d}=\mathbf{1 . 0} / T_{b}$ versus $I / S$ dB for MSK 


\subsubsection{Simulation Results}

In the literature, there is no standard set of parameters or criteria for evaluating ACI. Since there is a whole range of different possible parameters, from frequency spacing to relative power levels between SOI and the $\mathrm{ACI}$, it is difficult to cross reference other results from previous research in this area. For example, the DFE performance in ACI is typically given by the MMSE obtained, not the average bit error performance. One way to compare the two receivers is to approximately determine the threshold of ACI power that each receiver can endure before any serious performance degradation[24]. Typically receivers exhibit a threshold effect under $\mathrm{ACl}$, where when the $\mathrm{ACI}$ power is below the threshold there is negligible performance degraldition, whereas above this threshold there is rapid degradation. The threshold here is quintified as the Interference to Signal (I/S) power at which the receiver suffers $3 \mathrm{~dB}$ loss in performance at an average probability of bit error of $10^{-5}$.

The accuracy of the Gaussian model is approximately within $1 \mathrm{~dB}$ of the actual performance in $\mathrm{ACl}$ for the BTR as shown by Figures 4-5 to 4-22. In general, the Gaussian model gives a slightly over optimistic performance compared to the actual performance in ACI. The differences in performance can be accounted for by the fact the filtered ACI is not white and that there is statistical dependence between the quadrature branches. This approximate agreement between the Gaussian model and the actual performance indicates that modeling the filtered $\mathrm{ACI}$ as WGN is a reasonable assumption. There maybe additional benefit in exploring more accurate models for the BTR that 
account for the statistical dependence between samples as well as between each quadrature branch.

For the defined threshold of $3 \mathrm{~dB}$ Eb/No loss compared to the MF with no $\mathrm{ACI}$ at $\mathrm{Pe}=10^{-5}$, Tables III-V give this threshold for MF and BTR as derived from the probability of error plots .

Table III.

I/S dB threshold for OQPSK, and MSK with one interferer at $\mathrm{f}_{\mathrm{d}}$

\begin{tabular}{|c|c|c|c|}
\hline MF & $\mathbf{f}_{\mathbf{d}}=\mathbf{1 . 0} / \mathbf{T}_{\mathbf{b}}$ & $\mathbf{f}_{\mathrm{d}}=\mathbf{1 . 5} / \mathbf{T}_{\mathbf{b}}$ & $\mathbf{f}_{\mathbf{d}}=\mathbf{2 . 0} / \mathbf{T}_{\mathbf{b}}$ \\
\hline OQPSK & $0.7 \mathrm{~dB}$ & $4.2 \mathrm{~dB}$ & $6.8 \mathrm{~dB}$ \\
\hline MSK & $6.0 \mathrm{~dB}$ & $14.0 \mathrm{~dB}$ & $21.0 \mathrm{~dB}$ \\
\hline
\end{tabular}

Table IV.

$\mathrm{I} / \mathrm{S} \mathrm{dB}$ threshold for the BTR with $\mathrm{W}=0.5 / \mathrm{T}_{\mathrm{b}}$ for OQPSK and MSK with one interferer at $f_{d}$ and the difference $(\Delta)$ in $\mathrm{dB}$ to $M F$ for same $f_{d}$.

\begin{tabular}{|c|c|c|c|c|c|c|}
\hline $\mathrm{W}=0.5 / \mathbf{T}$ & $f_{d}=1.0 / T_{b}$ & $f_{d}=1.5 / T_{b}$ & $f_{d}=2.0 / T_{1}$ & $\mathrm{f}_{\mathrm{d}}=1.0 / \mathbf{T}_{\mathbf{b}}$ & $f_{d}=1.5 / T_{b}$ & $f_{d}=2.0 / T_{b}$ \\
\hline OQPSK & $3.5 \mathrm{~dB}$ & $7.6 \mathrm{~dB}$ & & & & $\mathrm{~dB}$ \\
\hline MSK & $8.9 \mathrm{~dB}$ & $18.8 \mathrm{~dB}$ & $24.5 \mathrm{~dB}$ & $\Delta 2.9 \mathrm{~dB}$ & $\Delta 4.8 \mathrm{~dB}$ & $\Delta 3.5 \mathrm{~dB}$ \\
\hline
\end{tabular}

Table $V$.

U/S dB Threshold for the BTR with $\mathrm{W}=0.25 / \mathrm{T}_{\mathrm{b}}$ for OQPSK and MSK with one interferer at $f_{d}$ and the difference $(\Delta)$ in $d B$ to MF for same $f_{d}$.

\begin{tabular}{|c|c|c|c|c|c|c|}
\hline$N=\mathbf{0 . 2 5} / \mathbf{T}_{1}$ & $f_{d}=1.0 / T_{b}$ & $f_{d}=1.5 / T_{b}$ & $f_{d}=2.0 / T_{11}$ & $f_{\mathrm{d}}=1.0 / T_{b}$ & $f_{d}=1.5 / T_{b}$ & \\
\hline OQPSK & $2.2 \mathrm{~dB}$ & $5.9 \mathrm{~dB}$ & & & & \\
\hline MSK & $8.0 \mathrm{~dB}$ & $15.1 \mathrm{~dB}$ & $20.9 \mathrm{~dB}$ & $\Delta 2.0 \mathrm{~dB}$ & $\Delta 1.1 \mathrm{~dB}$ & $-\Delta 0.1 \mathrm{dl}$ \\
\hline
\end{tabular}

The MF simulation results for MSK at $f_{d}=1.0 / T_{b}$ and $1.5 / T_{b}$, and for OQPSK at $f_{d}=1.0 / T_{b}$, agree very closely to the analytical results given in [24]. The BTR with $W=0.25 / T_{b}$ in general performs worse than with $W=0.5 / T_{b}$. Ass with the case of AWGN only, the BTR with a narrower bandwidth results in a net performance degradation even though the interference has also been narrowly filtered. 
The BTR with $\mathrm{W}=0.5 / \mathrm{T}_{\mathrm{b}}$ for both MSK and OQPSK shows more robustness in suppressing ACI than the MF. The gains in the threshold of above $2.8 \mathrm{~dB}$ for these modulations, from the close spacing of $1.0 / \mathrm{T}_{\mathrm{b}}, 102.0 / \mathrm{T}_{\mathrm{b}}$, compared to the MF can represent significant benefit. The gain of $4.8 \mathrm{~dB}$ for $\mathrm{MSK}$ at the frequency spacing of $1.5 / \mathrm{T}_{\mathrm{b}}$ particularly stands out. Of interest is the performance difference of OQPSK and MSK in ACI. The tighter PDS of MSK results in a difference in threshold for the BTR with $\mathrm{W}=0.5 / \mathrm{T}_{\mathrm{b}}$ of $5.4 \mathrm{~dB}$ at $\mathrm{f}_{\mathrm{d}}=1.0 / \mathrm{T}_{\mathrm{b}}$ to $14 \mathrm{~dB}$ at $\mathrm{f}_{\mathrm{d}}=2.0 / \mathrm{T}_{\mathrm{b}}$. There are clear spectral savings in using MSK compared to OQPSK for either the MF or the BTR. 


\section{CONCLUSION}

The thesis investigated the performance of the BTR in AWGN and ACI for MSK class modulations. Since JMLSE is undesirable because of excess hardware complexity, the receiver in AWGN and ACI must project the received signal onto a subspace. The choice of subspace should have a superior $\mathrm{S} / \mathrm{I}$ ratio to the matched filter, while having comparable performance to the MF in the case of no interference. A natural choice for unsynchronized FDMA systems is a bandlimited space for the SOI. The BTR performs a MLSE on this bandlimited subspace. The simulation results for the BTR with $\mathrm{W}=0.5 / \mathrm{T}_{\mathrm{b}}$ show that this approach can have comparable performance to the MF in AWGN, with improved performance in $\mathrm{ACl}$ from $2.8 \mathrm{~dB}$ to $4.8 \mathrm{~dB}$ for the cases studied. This approach could be applied to unsynchronized CDMA systems, where one possible subspace could be the same spreading code but with a diflerent pulse shape; one that emphasizes pulse transitions.

The BTR can be applied to bandwidth efficient non-linear modulation such as trellis coded modulation or continuous phase modulation[22]. An appropriate bandlimited space is chosen so as not to degrade the distances in the signal constellation, with the resulting intersymbol interference included in the signal's trellis. For these more bandwidth efficient modulations, such as 64 quadrature amplitude modulation TCM or quaternary partial response CPM, a narrower bandwidth than $0.5 / T_{b}$ may suffice. Note that the resulting ISI from narrowly filtered CPM cannot be frequency equalized in general, since the modulation is non-linear.

Another advantage for the BTR for bandwidth efficient modulations with many states in the trellis is to allow less complex sub-optimum trellis decoding without increased sensitivity to 
ACI. Simmons has shown that reduced state decoding of CPM with a matched filter front end is more sensitive to ACI than MLSE decoding of CPM with the VA [22]. The reduced state decoding causes early decisions between two signals before they have merged in the signal trellis. Thus unlike the VA case the difference signal is discontinuous which means that the spectral response of this reduced state decoding receiver rolls off at $1 / \mathrm{f}^{2}$. Hence this receiver is highly sensitive to ACI which has the same spectral roll off as OQPSK. The BTR however, already narrowly filters the ACI, which means that early decisions in the signal trellis are subject to less ACI power. In addition, since the filtered ACI is close 10 Gaussian, the performance of reduced state decoding in ACI for the BTR may be close to the performance in AWGN. 


\section{References}

1] G.S. Deshpande and P. H. Wittke, "Optimum pulse shaping in digital angle modulation” ,IEEE Trans. Commun.,vol. 29, pp. 162-169, Feb. 1981.

2]B.R. Peterson and D.D. Falconer, "Suppression of Adjacent-Channel, Cochannel, and Intersymbol Interference by Equalizers and Linear Combiners", IEEE Trans. Commun., vol. 42, pp. 3109-3118, Dec. 1994.

3] B.R. Peterson and D.D. Falconer, "Minimum Mean Square Equalization in Cyclostationary and

Stationary Interference-Analysis and Subscriber Line Calculations", IEEE Journal on Selected Areas in Communications, Vol. 9, pp 931-940, August 1991.

4]Harry L. Van Trees. Detection, Estimation, and Modualtion Theory- PART 1. John Wiley \& Sons, 1968. pp. 246-287

5] Harry L. Van Trees. Detection, Estimation, and Modualtion Theory- PART 1. John Wiley \& Sons, 1968. pp. 333-366

6]Gerhard Kramer in conversation in 1994. 
7]Sergio Verdu, "Minimum Probability of Error for Asynchronous Gaussian Multiple-Access Channels", IEEE Trans. on Information Theory, VOL. IT-32, pp. 85-96, Jan. 1986.

8]K. Giridhar, S. Chari, J. J. Shynk, and R. P. Gooch, "Joint demodulation of cochannel signals using MLSE and MAPSD algorithms", in Proc. IEEE Int. Conf. on Acoustics, Speech, and Signal Processing, pp. 640-655, April 1993.

9]R.Y. Hongui and I. Kalet, "MSK-Type Signals in the Presence of Adjacent Channel Interference and White Noise", to be published.

10]R.E. Eaves and S.M. Wheatley, "Optimization of Quadrature-Carrier Modulation for Low Crosstalk and Close Packing of Users", IEEE Trans. Commun, VOL. COM-27, pp. 176-185., Jan. 1979.

11]Irving Kalet, "A Look at Crosstalk in Quadrature-Carrier Modulation Systems", IEEE Trans. Commun, VOL. COM-25, pp. 884-892., Sept. 1977.

12]M. Abdulrahman and D.D Falconer, "Cyclostationary Crosstalk Suppression by Decision Feedback Equalization on Digital Subscriber Loops", IEEE Journal on Selected Areas in Communications, VOL. 10, pp. $640-648$. 
13]P. Niger and P. Vandamme, "Performance of Equalization Techniques in a Radio Interference Environment", IEEE Trans. Commun., VOL. 39, pp. 452-458, March 1991.

14]W. A. Gardner, "Cyclic Wiener Filtering: Theory and Method", IEEE Trans. Commun., VOL. 41, pp. 151-163, Jan. 1993.

15]W.A. Gardner, W. A. Brown III, and C.K. Chen, "Spectral Correlation of Modulated Signals: Part II- Digital Modulation", IEEE Trans. Commun.,VOL. COM-35, pp.595-601, June 1987.

16]M. Abdulrahman, A.U.H. Sheikh, and D.D. Falconer, "Decision Feedback Equalization for CDMA in Indoor Wireless Communications", IEEE Journal on Selected Areas in Comm., VOL. 12, pp. 698 - 705., May 1994.

17]John Proakis. Digital Communications. Second Edition. Mcgraw-Hill. 1989. p. 600.

18]G. D. Forney, "Maximum-Likelihood Sequence Estimation of Digital Sequences in the Presence of Intersymbol Interference “, Trans. on Information Theory, VOL. IT-18, pp.363-377, May 1972.

19] G. Ungerboeck, "Adaptive Maximum-Likelihood Receiver for Carrier-Modulated Data Transmission Systems “, IEEE Trans. Commun., VOL. COM-22, pp. 624-636, May 1974 
20]S.J Simmons, "Simplified Coherent Detection of Bandwidth-Efficient CPFSK/CPM Modulations", IEEE Pac.Rim. Conf. on CCSP, Victoria, B.C., May 1991, pp. 174-177.

21]S.J. Simmons, "ACI Susceptibility of Reduced-State Decoding for CPM, Biennial Sumposium on Communications -Queen's University, pp. 261-264, 1992.

22]S.J Simmons, "Simplified Coherent Detection of CPM", IEEE Trans. Commun., VOL. 43, pp. 726-728, Feb/March/April 1995.

23]J. R. Cruz, R. S. Simpson, "Cochannel and Intersymbol Interference in Quadrature-Carrier Modulation Systems”, IEEE Trans. Commun., VOL. 29, pp 285-297, March 1981.

24]R.B. Rhodes Jr, S. G. Wilson, and N.A.B. Svensson, "MSK-Type Reception of Continuous Phase Modulation: Cochannel and Adjacent Channel Interference", IEEE Trans. Commun., VOL. COM-35, pp. 185-193, Feb. 1987.

25]N.A.B. Svensson, "Error Probability Analysis for Continuous Phase Modulation with Viterbi Detection on a Gaussian Channel with Multiple Signal Interference", IEEE Trans. Commun., VOL. 37, pp. 230-237, March 1989. 